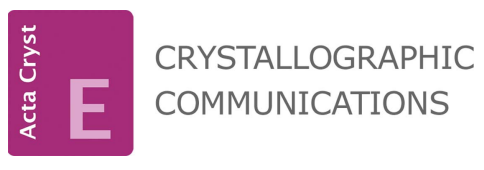

ISSN 2056-9890

Received 20 June 2017

Accepted 10 July 2017

Edited by M. Zeller, Purdue University, USA

Keywords: crystal structure; dithiolene; trigonal prismatic; oxalate.

CCDC references: 1561379; 1561378

Supporting information: this article has supporting information at journals.iucr.org/e

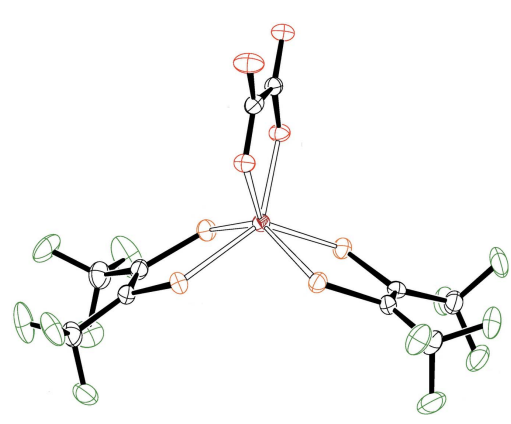

OPEN $\odot$ ACCESS

\section{Coordination compounds containing bis-dithiolene- chelated molybdenum(IV) and oxalate: comparison of terminal with bridging oxalate}

\author{
Agata Gapinska, ${ }^{a}$ Alan J. Lough ${ }^{\mathrm{b} *}$ and Ulrich Fekl ${ }^{\mathrm{a} *}$ \\ ${ }^{\mathbf{a}}$ Department of Chemical and Physical Sciences, University of Toronto, Mississauga, Ontario, L5L 1C6, Canada, and \\ ${ }^{\mathbf{b}}$ Department of Chemistry, 80 St George St., Toronto, ON, M5S 3H6, Canada. *Correspondence e-mail: \\ alough@chem.utoronto.ca, ulrich.fekl@utoronto.ca
}

Two coordination compounds containing tetra- $n$-butylammonium cations and bis-tfd-chelated molybdenum(IV) $\left[\mathrm{tfd}^{2-}=\mathrm{S}_{2} \mathrm{C}_{2}\left(\mathrm{CF}_{3}\right)_{2}{ }^{2-}\right]$ and oxalate $\left(\mathrm{ox}^{2-}\right.$, $\mathrm{C}_{2} \mathrm{O}_{4}{ }^{2-}$ ) in complex anions are reported, namely bis(tetra- $n$-butylammonium) bis(1,1,1,4,4,4-hexafluorobut-2-ene-2,3-dithiolato)oxalatomolybdate(IV)-chloroform-oxalic acid (1/1/1), $\left(\mathrm{C}_{16} \mathrm{H}_{36} \mathrm{~N}\right)_{2}\left[\mathrm{Mo}\left(\mathrm{C}_{4} \mathrm{~F}_{6} \mathrm{~S}_{2}\right)_{2}\left(\mathrm{C}_{2} \mathrm{O}_{4}\right)\right] \cdot \mathrm{CHCl}_{3} \cdot \mathrm{C}_{2} \mathrm{H}_{2} \mathrm{O}_{4}$ or $\left(\mathrm{N}^{n} \mathrm{Bu}_{4}\right)_{2}\left[\mathrm{Mo}(\mathrm{tfd})_{2}(\mathrm{ox})\right] \cdot \mathrm{CHCl}_{3} \cdot \mathrm{C}_{2} \mathrm{H}_{2} \mathrm{O}_{4}$, and bis(tetra- $n$-butylammonium) $\mu$-oxalato-bis[bis(1,1,1,4,4,4-hexafluorobut-2-ene-2,3-dithiolato)molybdate(IV)], $\left(\mathrm{C}_{16} \mathrm{H}_{36} \mathrm{~N}\right)_{2}\left[\mathrm{Mo}_{2}\left(\mathrm{C}_{4} \mathrm{~F}_{6} \mathrm{~S}_{2}\right)_{4}\left(\mathrm{C}_{2} \mathrm{O}_{4}\right)\right]$ or $\left(\mathrm{N}^{n} \mathrm{Bu}_{4}\right)_{2}\left[(\mathrm{tfd})_{2} \mathrm{Mo}(\mu\right.$-ox $\left.) \mathrm{Mo}(\mathrm{tfd})_{2}\right]$. They contain a terminal oxalate ligand in the first compound and a bridging oxalate ligand in the second compound. Anion $\mathbf{1}^{2-}$ is $\left[\mathrm{Mo}(\mathrm{tfd})_{2}(\mathrm{ox})\right]^{2-}$ and anion $\mathbf{2}^{2-}$, formally generated by adding a $\mathrm{Mo}(\mathrm{tfd})_{2}$ fragment onto $\mathbf{1}^{2-}$, is $\left[(\mathrm{tfd})_{2} \mathrm{Mo}(\mu-\right.$ ox) $\left.\mathrm{Mo}(\mathrm{tfd})_{2}\right]^{2-}$. The crystalline material containing $\mathbf{1}^{2-}$ is $\left(\mathrm{N}^{n} \mathrm{Bu}_{4}\right)_{2^{-}}$ 1. $\mathrm{CHCl}_{3} \cdot \mathrm{OxH}_{2}$, while the material containing $\mathbf{2}^{2-}$ is $\left(\mathrm{N}^{n} \mathrm{Bu}_{4}\right)_{2}-\mathbf{2}$. Anion $\mathbf{2}^{2-}$ lies across an inversion centre. The complex anions afford a rare opportunity to compare terminal oxalate with bridging oxalate, coordinated to the same metal fragment, here $(\mathrm{tfd})_{2} \mathrm{Mo}^{\mathrm{IV}}$. $\mathrm{C}-\mathrm{O}$ bond-length alternation is observed for the terminal oxalate ligand in $\mathbf{1}^{2-}$ : the difference between the $\mathrm{C}-\mathrm{O}$ bond length involving the metal-coordinating $\mathrm{O}$ atom and the $\mathrm{C}-\mathrm{O}$ bond length involving the uncoordinating $\mathrm{O}$ atom is $0.044(12) \AA$. This bond-length alternation is significant but is smaller than the bond-length alternation observed for oxalic acid in the co-crystallized oxalic acid in $\left(\mathrm{N}^{n} \mathrm{Bu}_{4}\right)_{2}-\mathbf{1} \cdot \mathrm{CHCl}_{3} \cdot \mathrm{OxH}_{2}$, where a difference (for $\mathrm{C}=\mathrm{O}$ versus $\mathrm{C}-\mathrm{OH}$ ) of 0.117 (14) $\AA$ was observed. In the bridging oxalate ligand in $\mathbf{2}^{2-}$, the $\mathrm{C}-\mathrm{O}$ bond lengths are equalized, within the error margin of one bond-length determination $(0.006 \AA)$. It is concluded that oxalic acid contains a localized $\pi$-system in its carboxylic acid groups, that the bridging oxalate ligand in $\mathbf{2}^{2-}$ contains a delocalized $\pi$-system and that the terminal oxalate ligand in $\mathbf{1}^{2-}$ contains an only partially localized $\pi$-system. In $\left(\mathrm{N}^{n} \mathrm{Bu}_{4}\right)_{2}-\mathbf{1} \cdot \mathrm{CHCl}_{3} \cdot \mathrm{OxH}_{2}$, the $\mathrm{F}$ atoms of two of the $-\mathrm{CF}_{3}$ groups in $\mathbf{1}^{2-}$ are disordered over two sets of sites, as are the $\mathrm{N}$ and eight of the $\mathrm{C}$ atoms of one of the $\mathrm{N}^{n} \mathrm{Bu}_{4}$ cations. In $\left(\mathrm{N}^{n} \mathrm{Bu}_{4}\right)_{2}-2$, the whole of the unique $\mathrm{N}^{n} \mathrm{Bu}_{4}{ }^{+}$cation is disordered over two sets of sites. Also, in $\left(\mathrm{N}^{n} \mathrm{Bu}_{4}\right)_{2}-\mathbf{2}$, a region of disordered electron density was treated with the SQUEEZE routine in PLATON [Spek (2015). Acta Cryst. C71, 9-18].

\section{Chemical context}

The oxalate $\left(\mathrm{ox}^{2-}, \mathrm{C}_{2} \mathrm{O}_{4}{ }^{2-}\right)$ ion is a very useful ligand in transition metal chemistry. Its usefulness stems in part from its ability to act as a chelate ligand toward a metal cation while retaining two more $\mathrm{O}$ atoms with the ability to donate to another metal cation. Thus, while coordination compounds containing terminal oxalate are known, oxalates can easily act as bridging ligands to allow for the synthesis of dimetallic and multimetallic molecular compounds, as well as extended 


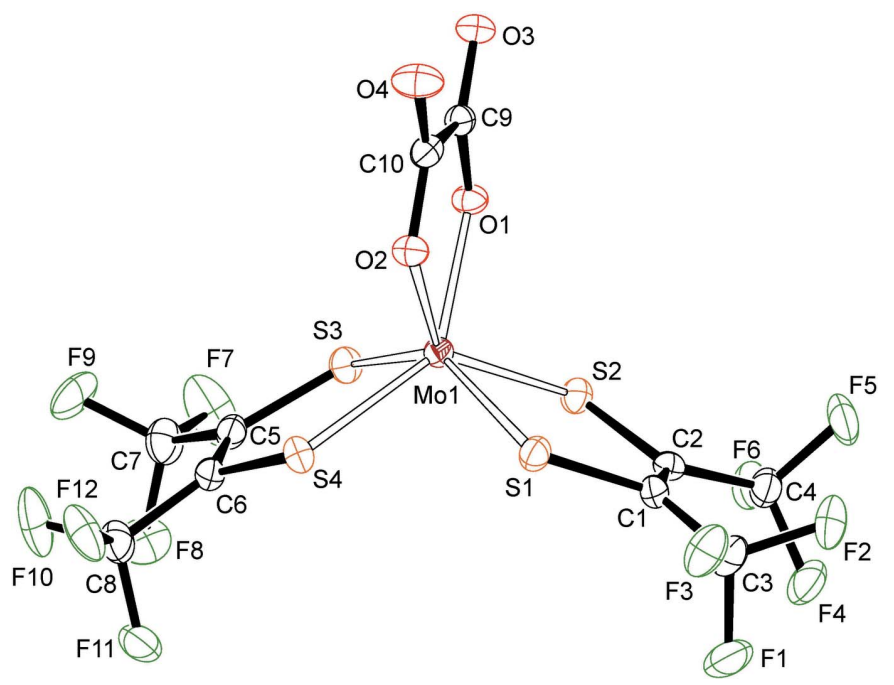

Figure 1

A view of the molecular structure of $\mathbf{1}^{2-}$ in $\left(\mathrm{N}^{n} \mathrm{Bu}_{4}\right)_{2}-\mathbf{1} \cdot \mathrm{CHCl}_{3} \cdot \mathrm{oxH}_{2}$. Anisotropic displacement ellipsoids are shown at the $30 \%$ probability level.

coordination polymers (Clemente-León et al., 2011; Gruselle et al., 2006). Most of the work has involved V, Cr, Mn, Fe, Co, $\mathrm{Ni}$ and $\mathrm{Cu}$, as well as $\mathrm{Ru}$ and $\mathrm{Rh}$. Compounds where oxalate coordinates to molybdenum are rare, although some examples have been synthesized, mostly in the context of nitrogenase models, where oxalate was deemed a model for homocitrate (Demadis \& Coucouvanis, 1995). Stimulated by our previous results on the molybdenum(IV) dithiolene fragment $\mathrm{Mo}(\mathrm{tfd})_{2}$ $\left[\mathrm{tfd}^{2-}=\mathrm{S}_{2} \mathrm{C}_{2}\left(\mathrm{CF}_{3}\right)_{2}{ }^{2-}\right]$ with a labile 'cap' (Harrison et al., 2007; Nguyen et al., 2010), we added oxalate to the $\mathrm{Mo}(\mathrm{tfd})_{2}$ fragment, as described in the 'Synthesis and crystallization' section
Figure 2

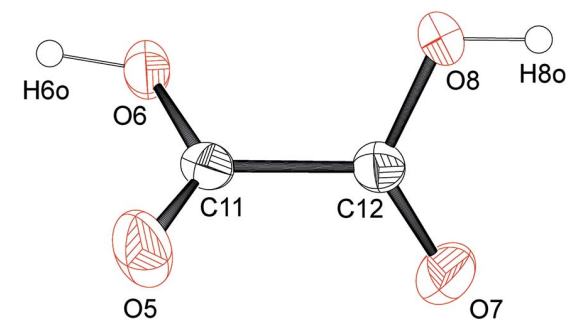

A view of the molecular structure of the oxalic acid $\left(\mathrm{oxH}_{2}\right)$ molecule in $\left(\mathrm{N}^{n} \mathrm{Bu}_{4}\right)_{2}-\mathbf{1} \cdot \mathrm{CHCl}_{3} \cdot \mathrm{oxH}_{2}$. Anisotropic displacement ellipsoids are shown at the $30 \%$ probability level.

$(\S 5)$. The $\left[\mathrm{Mo}(\mathrm{tfd})_{2}(\mathrm{ox})\right]^{2-}\left(\mathbf{1}^{2-}\right)$ and $\left[(\mathrm{tfd})_{2} \mathrm{Mo}(\mu\right.$-ox $) \mathrm{Mo-}$ $\left.(\mathrm{tfd})_{2}\right]^{2-}\left(\mathbf{2}^{2-}\right)$ anions were indeed obtained, offering an opportunity for a structural comparison.

\section{Structural commentary}

The counter-cation for both complex molybdate anions was tetra- $n$-butylammonium. $\mathbf{1}^{2-}$ was obtained as $\left(\mathrm{N}^{n} \mathrm{Bu}_{4}\right)_{2^{-}}$ 1. $\mathrm{CHCl}_{3} \cdot \mathrm{OxH}_{2}$, while $\mathbf{2}^{2-}$ was obtained as $\left(\mathrm{N}^{n} \mathrm{Bu}_{4}\right)_{2}-\mathbf{2}$. The molecular structure of $\mathbf{1}^{2-}$ is shown in Fig. 1 , where $\mathrm{N}^{n} \mathrm{Bu}_{4}{ }^{+}$ counter-ions and co-crystallized oxalic acid, as well as chloroform solvent molecules, are not shown. Only one orientiation is shown for the disordered trifluoromethyl groups involving atoms C7 and C8. The charge on the molybdenum-containing moiety, which is identified as $\mathbf{1}^{2-}$, is unambiguous, due to the tetra- $n$-butylammonium cations. While tfd can be redox-non-innocent (Hosking et al., 2009), it is redox-innocent here. The $\mathrm{C}-\mathrm{C}$ bond lengths in the two tfd ligand backbones $[1.349$ (8) $\AA$ for $\mathrm{C} 1-\mathrm{C} 2$ and 1.353 (8) $\AA$ for

Figure 3

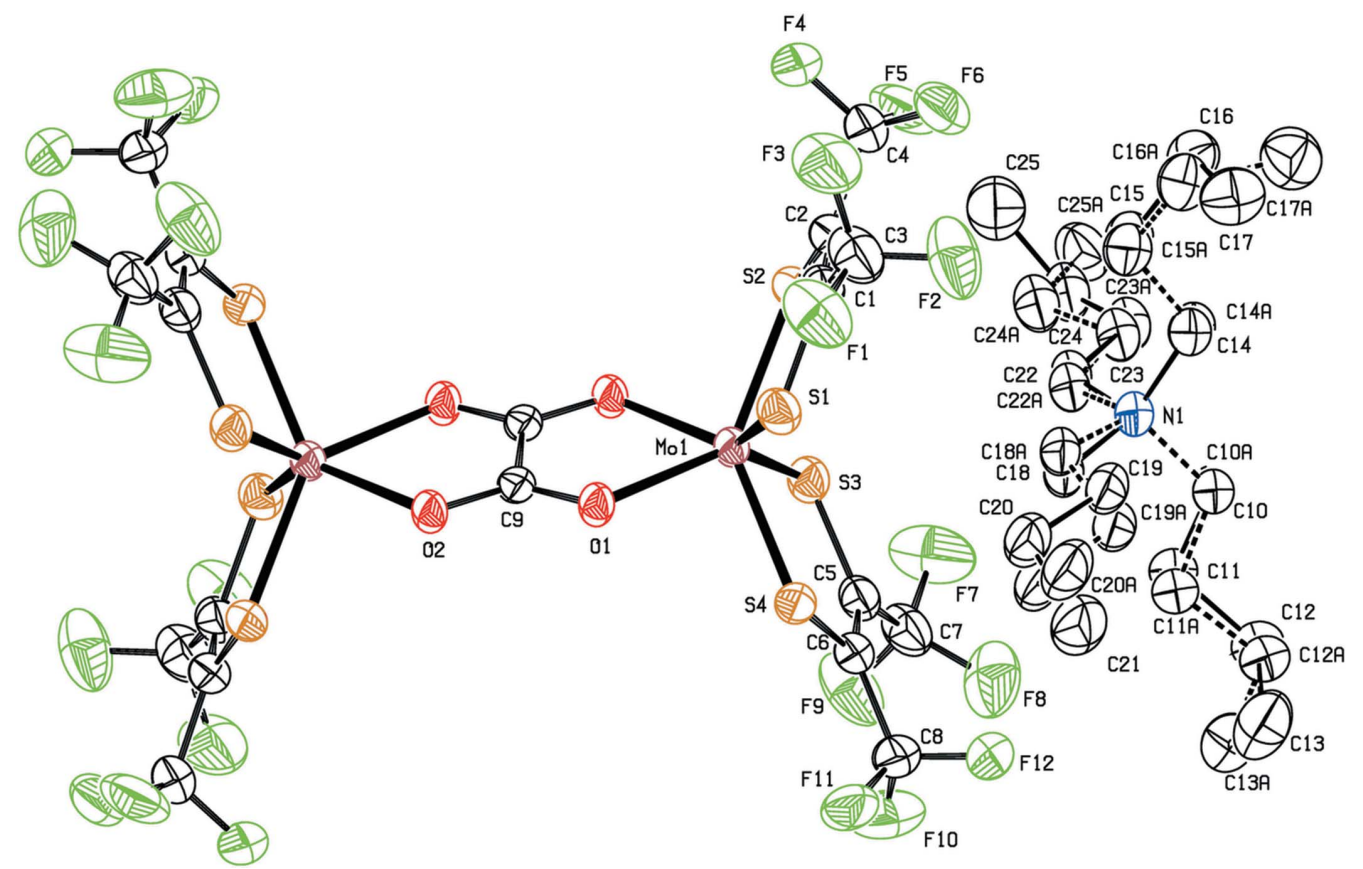

A view showing the $\mathbf{2}^{2-}$ anion and the (disordered) $\mathrm{N}^{n} \mathrm{Bu}_{4}{ }^{+}$cation in $\left(\mathrm{N}^{n} \mathrm{Bu}_{4}\right)_{2}-\mathbf{2}$. Anisotropic displacement ellipsoids are shown at the $30 \%$ probability level. The minor component of disorder is shown with dashed bonds. Unlabelled atoms are related by a crystallographic inversion centre (symmetry code: $-x+2,-y,-z+1)$. 

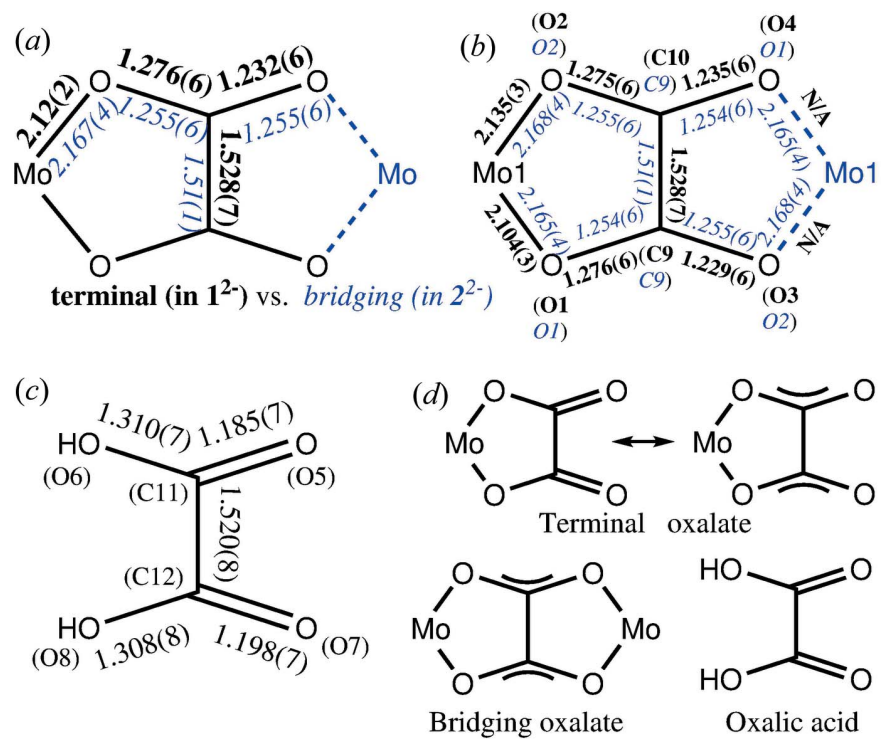

Figure 4

Bond-length changes on going from terminal to bridging oxalate, summarized $(a)$ and in detail $(b)$, as well as bond lengths in the oxalic acid molecule observed $(c)$ and concluding resonance description $(d)$.

C5-C6] are a clear indication of fully reduced (dianionic) ene-dithiolate $\left(\mathrm{tfd}^{2-}\right)$, such that the oxidation state of the metal is +IV. The Mo-S bond lengths, ranging from 2.3265 (14) to 2.3390 (15) $\AA$, are as expected for tfd complexes of $\mathrm{Mo}^{\text {IV }}$ (Nguyen et al., 2010). Regarding the bonded oxalate, the average $\mathrm{Mo}-\mathrm{O}$ bond length is $2.12 \AA$ [Mo1-O1 = 2.104 (3) $\AA$ and Mo1-O2 = 2.135 (3) $\AA$ ]. Within the oxalate unit, the chemically distinct $\mathrm{O}$ atoms (coordinating to molybdenum versus uncoordinating) show different bond lengths to the directly bonded $\mathrm{C}$ atom. The $\mathrm{C}-\mathrm{O}$ bond length involving the metal-coordinating $\mathrm{O}$ atom is $1.276(6) \AA$ (average of two values), with the $\mathrm{C}-\mathrm{O}$ bond length involving the uncoordinating $\mathrm{O}$ atom is 1.232 (6) $\AA$ (average of two values), for a difference of 0.044 (12) $\AA$. While it may be tempting to describe the longer $\mathrm{C}-\mathrm{O}$ bond as a single bond and the shorter $\mathrm{C}-\mathrm{O}$ bond as a double bond, such a description would not be fully accurate since the bond-length alternation is only partial and less pronounced than for oxalic acid. The oxalic acid $\left(\mathrm{oxH}_{2}\right)$ molecule found in the structure of $\left(\mathrm{N}^{n} \mathrm{Bu}_{4}\right)_{2}-\mathbf{1} \cdot \mathrm{CHCl}_{3} \cdot \mathrm{OxH}_{2}$ is shown in Fig. 2. This oxalic acid molecule exhibits stronger bond-length alternation: a difference (for $\mathrm{C}=\mathrm{O}$ versus $\mathrm{C}-\mathrm{OH}$ ) of 0.117 (14) $\AA$ is observed. For further comparison, the structure of $2^{2-}$, in $\left(\mathrm{N}^{n} \mathrm{Bu}_{4}\right)_{2}-\mathbf{2}$, is valuable. Both $\mathbf{2}^{2-}$ and the (disordered) tetra- $n$-butylammonium ion in the structure of $\left(\mathrm{N}^{n} \mathrm{Bu}_{4}\right)_{2}-2$ are shown in Fig. 3. For the bridging oxalate ligand in $\mathbf{2}^{2-}$, bond-length equalization is observed, within the error margin of one bondlength determination $(0.006 \AA)$. The details of the oxalate substructure are shown in Fig. 4, where Fig. 4(a) highlights the bond-length changes on going from a terminal oxalate in $\mathbf{1}^{2-}$ to a bridging oxalate in $\mathbf{2}^{2-}$, where parameters related to chemically equivalent bonds are averaged for clarity, and Fig. 4(b) shows all data before averaging. Fig. 4(c) shows the bond lengths in the free oxalic acid molecule in $\left(\mathrm{N}^{n} \mathrm{Bu}_{4}\right)_{2}$ -
1. $\mathrm{CHCl}_{3} \cdot \mathrm{oxH}_{2}$. Fig. $4(d)$ summarizes the findings: oxalic acid contains a localized $\pi$-system in its carboxylic acid groups, the bridging oxalate in $\mathbf{2}^{2-}$ contains a delocalized $\pi$-system and terminal oxalate in $\mathbf{1}^{2-}$ contains a partially localized $\pi$-system. While only marginally significant ( $c a 1 \sigma$ ), an effect involving the $\mathrm{C}-\mathrm{C}$ bonds of oxalate can be seen: upon becoming bridging, the oxalate $\mathrm{C}-\mathrm{C}$ bond shortens from 1.528 (7) $\AA$ to 1.51 (1) $\AA$ (Figs. $4 a$ and $4 b$ ). While this bond shortening may initially be surprising, it is actually theoretically expected: the $\pi$-system in a localized butadiene-like system is antibonding with respect to the central $\mathrm{C}-\mathrm{C}$ bond. When oxalate becomes bridging, due to delocalization in the $\pi$-system, the electronic structure is no longer butadiene-like but rather resembles two allyl anions linked at the central $\mathrm{C}$ atom, where the $\pi$-overlap at the central $\mathrm{C}$ atoms is not antibonding but just nonbonding. Apart from the specifics of the oxalate substructure in $\mathbf{2}^{2-}$, there are no dramatic changes in the coordination sphere of molybdenum on going from $\mathbf{1}^{2-}$ to $\mathbf{2}^{2-}$. The points made above for $\mathbf{1}^{2-}$ related to $\mathrm{Mo}-\mathrm{S}$ bond lengths (normal) and $\mathrm{C}-\mathrm{C}$ bond lengths in the tfd ligand (double bond) typically apply also to $\mathbf{2}^{2-}$. Also, both metal centres much more closely resemble a trigonal prismatic structure than an octahedral structure, as is expected for $d^{2}$ tris-chelates involving dithiolenes. Using the $X-M-X_{\text {trans }}$ criterion (Beswick et al., 2004; Nguyen et al., 2010), the geometry around molybdenum in $\mathbf{1}^{2-}$ is $88 \%$ trigonal-prismatic. Using the same method, the geometry around molybdenum in $\mathbf{2}^{2-}$ analyzes as $99 \%$ trigonal-prismatic.
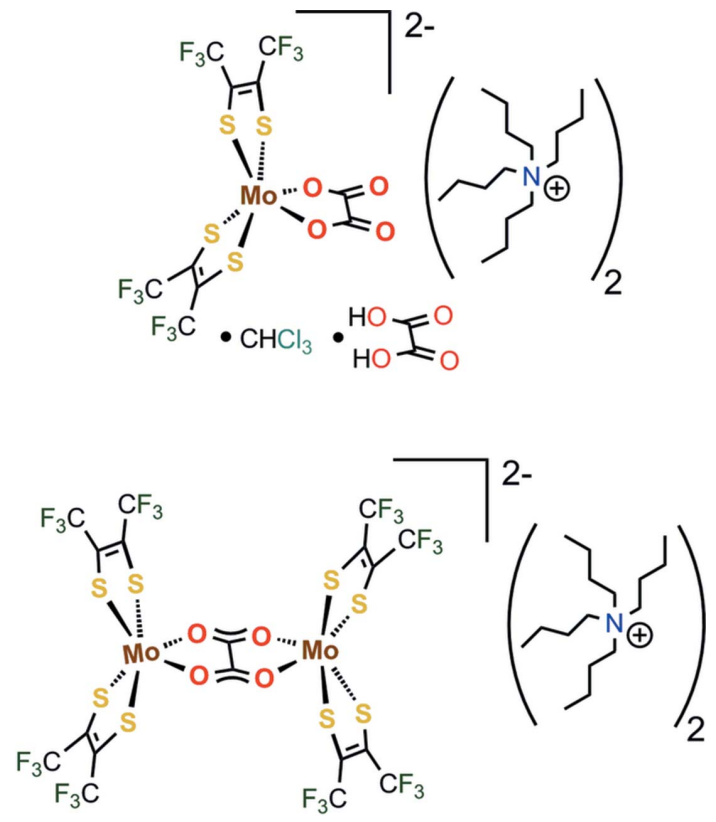

\section{Supramolecular features}

The oxalic acid solvent molecule and the metal-coordinating oxalate ligand in $\left(\mathrm{N}^{n} \mathrm{Bu}_{4}\right)_{2}-\mathbf{1} \cdot \mathrm{CHCl}_{3} \cdot \mathrm{oxH}_{2}$ form a hydrogenbonded network (Table 1 ). The oxalate $\mathrm{O}$ atoms of $\mathbf{1}^{2-}$ that are not metal coordinating act as hydrogen-bond acceptors. Oxalic acid acts as a hydrogen-bond donor: both of its $\mathrm{OH}$ functionalities hydrogen bond to two different molecules of $\mathbf{1}^{2-}$, such that infinite chains along [100] of the type ' $-\mathbf{1}^{2-}-$ 
Table 1

Hydrogen-bond geometry $\left(\AA,^{\circ}\right)$ for $\left(\mathrm{N}^{n} \mathrm{Bu}_{4}\right)_{2}-\mathbf{1} \cdot \mathrm{CHCl}_{3} \cdot \mathrm{oxH}_{2}$.

\begin{tabular}{lllll}
\hline$D-\mathrm{H} \cdots A$ & $D-\mathrm{H}$ & $\mathrm{H} \cdots A$ & $D \cdots A$ & $D-\mathrm{H} \cdots A$ \\
\hline $\mathrm{O} 6-\mathrm{H} 6 \mathrm{O} \cdots \mathrm{O}^{\mathrm{i}}$ & $0.88(7)$ & $1.76(7)$ & $2.633(5)$ & $170(7)$ \\
$\mathrm{O} 8-\mathrm{H} 8 \mathrm{O} \cdots \mathrm{O} 4$ & $0.85(8)$ & $1.75(8)$ & $2.587(5)$ & $174(9)$ \\
\hline
\end{tabular}

Symmetry code: (i) $x+\frac{1}{2},-y+\frac{3}{2},-z+1$.

HOOC-COOH $-\mathbf{1}^{2-}-$, etc' are formed. The $\left(\mathrm{N}^{n} \mathrm{Bu}_{4}\right)_{2}^{+}$ cations (one of them containing disorder) are packed around the $\mathbf{1}^{2-}$ anion, along with a $\mathrm{CHCl}_{3}$ solvent molecule that forms part of the structure. A plot showing anisotropic displacement ellipsoids for all non- $\mathrm{H}$ atoms (including disordered ones) in $\left(\mathrm{N}^{n} \mathrm{Bu}_{4}\right)_{2}-\mathbf{1} \cdot \mathrm{CHCl}_{3} \cdot \mathrm{OxH}_{2}$ is shown in Fig. 5. In contrast, there are no hydrogen bonds or notable close contacts in the structure of $\left(\mathrm{N}^{n} \mathrm{Bu}_{4}\right)_{2}-\mathbf{2}$, which consists of a packing of $\mathbf{2}^{2-}$ anions and $\mathrm{N}^{n} \mathrm{Bu}_{4}{ }^{+}$cations, both of which are shown in Fig. 3 .

\section{Database survey}

Relevant coordination compounds containing dithiolenes are discussed above, where review articles for coordinating oxalate are also referenced. A search of the Cambridge Structural Database (Version 5.38, including updates up to May 2017; Groom et al., 2016) reveals no reports of molybdenum dithiolene complexes that contain oxalate.

\section{Synthesis and crystallization}

\subsection{General specifications}

All manipulations involving metal-containing compounds were carried out under an inert $\left(\mathrm{N}_{2}\right)$ atmosphere using standard glove-box (M. Braun UniLab) and Schlenk techniques. Solvents were purified prior to use by vacuum distillation from molecular sieves. Organic and inorganic starting materials were obtained from Sigma-Aldrich. Mo(tfd $)_{2}(\text { tht })_{2}$ (tht $=$ tetrahydrothiophene) was synthesized from $\mathrm{Mo}(\mathrm{tfd})_{2}(\mathrm{bdt})$ (bdt $=\mathrm{S}_{2} \mathrm{C}_{6} \mathrm{H}_{4}$ ) as in Nguyen et al. (2010). $\mathrm{Mo}(\mathrm{tfd})_{2}$ (bdt) was synthesized as in Harrison et al. (2007). Tetra- $n$-butylammonium oxalate was prepared by neutralizing oxalic acid with aqueous tetrabutylammonium hydroxide, followed by drying under vacuum at $333 \mathrm{~K}$.

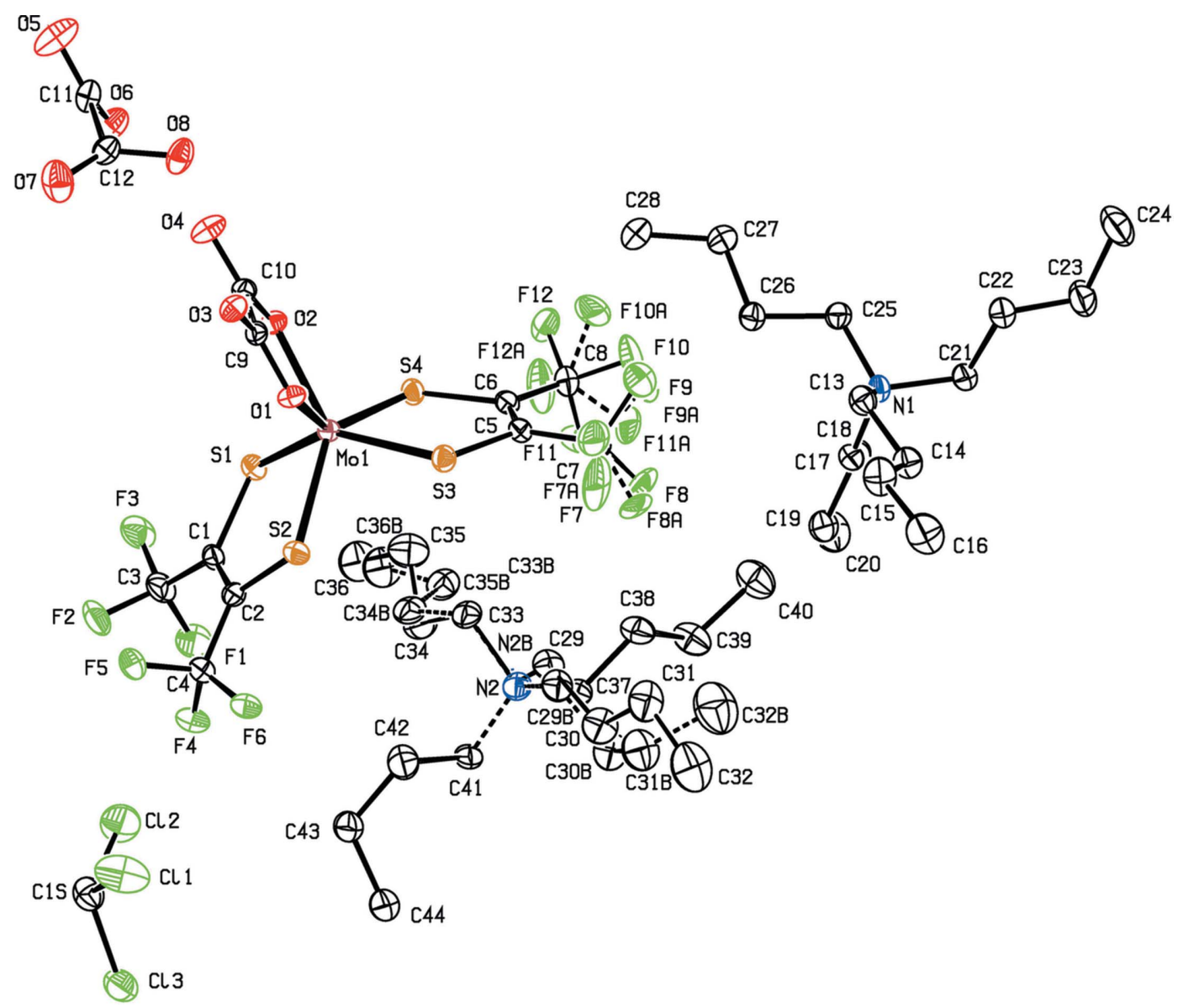

Figure 5

Anisotropic displacement plot (30\% probability level) showing all non-H atoms (including disordered ones and those of chloroform solvent) in $\left(\mathrm{N}^{n} \mathrm{Bu}_{4}\right)_{2}-\mathbf{1} \cdot \mathrm{CHCl}_{3} \cdot \mathrm{oxH}_{2}$. The minor component of disorder is shown with dashed bonds. Atom $\mathrm{N} 2$ is disordered over two sites and the major component is obscured by the minor component. 
Table 2

Experimental details.

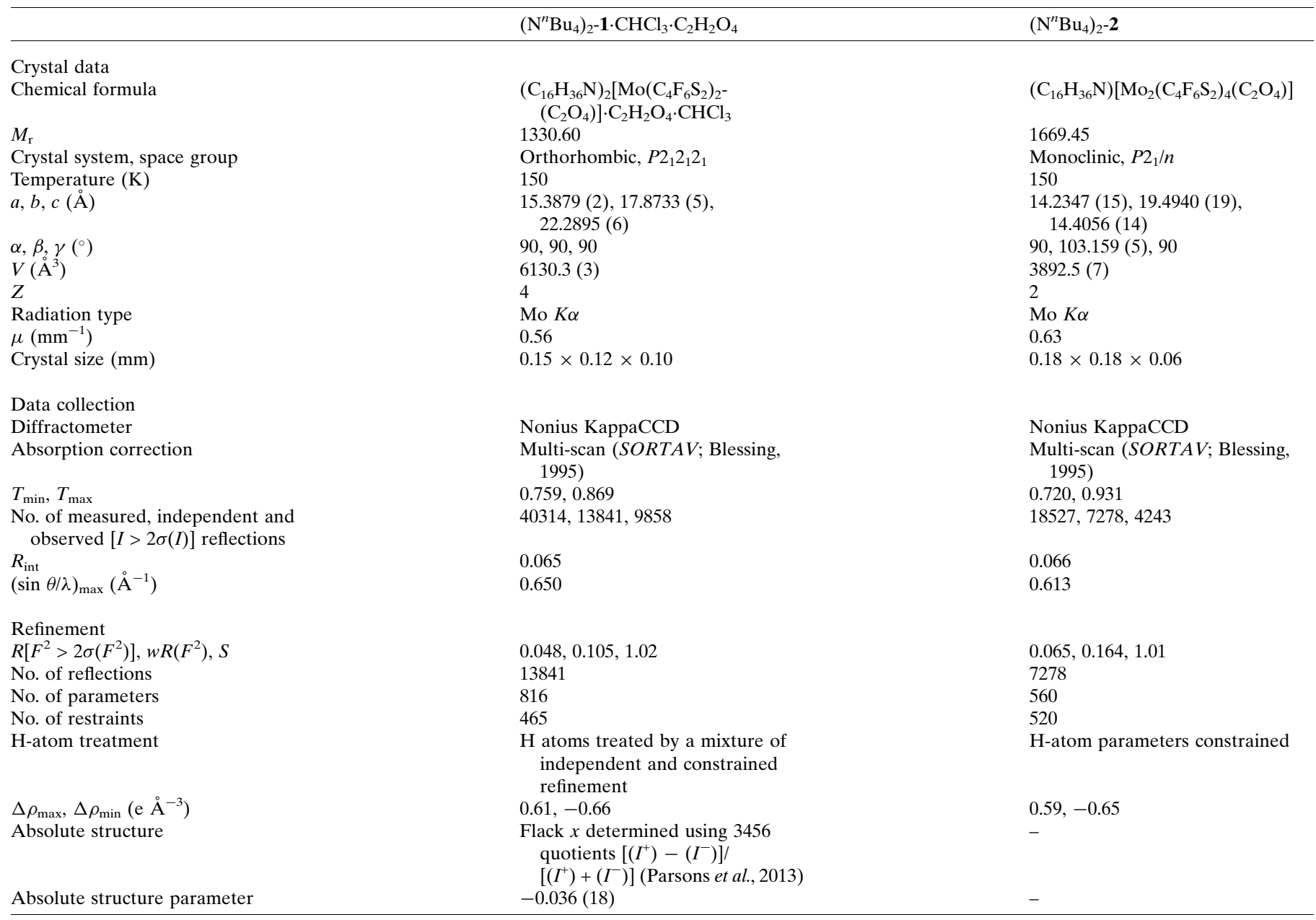

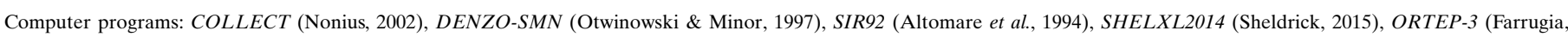
2012), PLATON (Spek, 2009) and SHELXTL (Sheldrick, 2008).

\subsection{Synthesis of $\left(\mathrm{N}^{n} \mathrm{Bu}_{4}\right)_{2}-1 \cdot \mathrm{CHCl}_{3} \cdot \mathrm{oxH}_{2}$}

We were unable to obtain $\mathbf{1}^{2-}$ as the only molybdenum product produced in a reaction. Attempts always led to significant decomposition to form a blue material, almost certainly molybdenum that is reduced below the oxidation state $+\mathrm{IV}$ due to the reducing power of oxalate. However, $\mathbf{1}^{2-}$ can be obtained as crystals (co-crystals with oxalic acid and chloroform) in the form of brown blocks. $2 \mathrm{mg}$ of $\mathrm{Mo}(\mathrm{tfd})_{2}(\mathrm{bdt})(2.9 \mu \mathrm{mol})$ were dissolved in a small amount of chloroform in a glass vial. In a second glass vial, $16.7 \mathrm{mg}$ $(29 \mu \mathrm{mol})$ of tetra- $n$-butylammonium oxalate were dissolved in the amount of chloroform needed to create a clear solution. The contents of the two vials were mixed and $3.3 \mu \mathrm{l}$ (14.6 $\mu \mathrm{mol})$ of bis(trimethylsilyl)acetylene, needed to labilize the bdt fragment (Nguyen et al., 2010), were added via microlitre syringe. The initially dark (blue-green) solution became lighter, and small brown particles began to form. After $72 \mathrm{~h}$, the solvent was reduced under vacuum, and orange-brown crystals grew. Blue-green needles (not of X-ray quality) of a different (likely reduced) molybdenum product were also growing. The orange-brown blocks were manually separated and chosen for X-ray crystallography.

\subsection{Synthesis of $\left(\mathrm{N}^{n} \mathrm{Bu}_{4}\right)_{2}-2$}

$2 \mathrm{mg}(2.8 \mu \mathrm{mol})$ of $\mathrm{Mo}(\mathrm{tfd})_{2}(\mathrm{tht})_{2}$ were dissolved in a minimal amount of chloroform. A solution of $16 \mathrm{mg}(28 \mu \mathrm{mol})$ of tetra- $n$-butylammonium oxalate in $2 \mathrm{ml}$ of chloroform was added. The solution turned red and, after $2 \mathrm{~h}$, thin pink rectangular crystals had formed. The liquid was decanted and the crystals were washed twice with chloroform and dried under vacuum. X-ray-quality crystals were grown using vapour diffusion. In a small vial, the product was dissolved in dichloromethane. The small vial was placed uncapped into a larger vial with chloroform. The larger vial was capped, and over a period of $2 \mathrm{~d}$, the dichloromethane solvent had evaporated from the small vial and dissolved in the chloroform in the larger vial, leaving pink crystals in the smaller vial. The crystals were found to be very air-sensitive, and exposure to air leads to decomposition to form a liquid that colours the surface of the crystals initially green and later blue. 


\section{Refinement}

Crystal data, data collection and structure refinement details are summarized in Table 2. In $\left(\mathrm{N}^{n} \mathrm{Bu}_{4}\right)_{2}-\mathbf{1} \cdot \mathrm{CHCl}_{3} \cdot \mathrm{oxH}_{2}, \mathrm{H}$ atoms bonded to $\mathrm{C}$ atoms were placed in calculated positions and included in a riding-motion approximation, while $\mathrm{H}$ atoms bonded to $\mathrm{O}$ atoms were refined independently with isotropic displacement parameters. In the anion $\mathbf{1}^{2-}$, atoms F7/F8/F9 were included as disordered over two sets of sites, with refined occupancies of 0.58 (2) and 0.42 (2). Atoms F10/F11/F12 were included as disordered, with refined occupancies of 0.502 (10) and 0.498 (10). The $\mathrm{C}-\mathrm{F}$ bond lengths and $\mathrm{F} \cdots \mathrm{F}$ distances were restrained using the SADI command in SHELXL (Sheldrick, 2015) and the anisotropic displacement parameters of the disordered $\mathrm{F}$ atoms and bonded $\mathrm{C}$ atoms were restrained using the SIMU command. In addition, the $\mathrm{N}$ and $8 \mathrm{C}$ atoms (C29-C36) of one of the independent $\mathrm{N}^{n} \mathrm{Bu}_{4}{ }^{+}$ cations were refined as disordered over two sets of sites, with refined occupancies of 0.676 (9) and 0.324 (9). The SAME command in SHELXL was used to restrain the geometry of the disordered C-atom chains to those of the ordered $\mathrm{N}^{n} \mathrm{Bu}_{4}{ }^{+}$ cation and the SIMU command was used to restrain anisotropic displacement parameters of the disordered atoms. In $\left(\mathrm{N}^{n} \mathrm{Bu}_{4}\right)_{2}-\mathbf{2}$, all $\mathrm{H}$ atoms were placed in calculated positions and refined in a riding-motion approximation. During the refinement of the structure of $\left(\mathrm{N}^{n} \mathrm{Bu}_{4}\right)_{2}-2$, electron-density peaks were located that were believed to be highly disordered solvent molecules (crystallization solvents were $\mathrm{CH}_{2} \mathrm{Cl}_{2} /$ $\mathrm{CHCl}_{3}$ ). Attempts made to model the solvent molecule were not successful. The SQUEEZE (Spek, 2015) option in PLATON (Spek, 2009) indicated that there was a large solvent cavity of $156 \AA$. In the final cycles of refinement, this contribution of 62.6 electrons to the electron density was removed from the observed data. The density, the $F(000)$ value, the molecular weight and the formula are given without taking into account the results obtained with the SQUEEZE option. Similar treatments of disordered solvent molecules were carried out by Stähler et al. (2001), Cox et al. (2003), Mohamed et al. (2003) and Athimoolam et al. (2005). Also in $\left(\mathrm{N}^{n} \mathrm{Bu}_{4}\right)_{2}-2$, the whole molecule of the unique $\mathrm{N}^{n} \mathrm{Bu}_{4}{ }^{+}$cation was included as disordered over two sets of sites, with refined occupancies of 0.589 (6) and 0.411 (6). The same command in SHELXL was used to restrain the geometry of the minor component of disorder to that of the major component and the
SIMU command was used to restrain all anisotropic diplacement parameters of the disordered atoms.

\section{Acknowledgements}

We thank Daniel J. Harrison and Neilson Nguyen (U of T) for providing $\mathrm{Mo}(\mathrm{tfd})_{2}(\mathrm{bdt})$ and $\mathrm{Mo}(\mathrm{tfd})_{2}(\text { tht })_{2}$.

\section{Funding information}

Funding for this research was provided by: Natural Sciences and Engineering Research Council of Canada; University of Toronto.

\section{References}

Altomare, A., Cascarano, G., Giacovazzo, C., Guagliardi, A., Burla, M. C., Polidori, G. \& Camalli, M. (1994). J. Appl. Cryst. 27, 435.

Athimoolam, S., Kumar, J., Ramakrishnan, V. \& Rajaram, R. K. (2005). Acta Cryst. E61, m2014-m2017.

Beswick, C. L., Schulman, J. M. \& Stiefel, E. I. (2004). Prog. Inorg. Chem. 52, 55-110.

Blessing, R. H. (1995). Acta Cryst. A51, 33-38.

Clemente-León, M., Coronado, E., Martí-Gastaldo, C. \& Romero, F. M. (2011). Chem. Soc. Rev. 40, 473-497.

Cox, P. J., Kumarasamy, Y., Nahar, L., Sarker, S. D. \& Shoeb, M. (2003). Acta Cryst. E59, o975-o977.

Demadis, K. D. \& Coucouvanis, D. (1995). Inorg. Chem. 34, 436-448.

Farrugia, L. J. (2012). J. Appl. Cryst. 45, 849-854.

Groom, C. R., Bruno, I. J., Lightfoot, M. P. \& Ward, S. C. (2016). Acta Cryst. B72, 171-179.

Gruselle, M., Train, C., Boubekeur, K., Gredin, P. \& Ovanesyan, N. (2006). Coord. Chem. Rev. 250, 2491-2500.

Harrison, D. J., Lough, A. J., Nguyen, N. \& Fekl, U. (2007). Angew. Chem. Int. Ed. 46, 7644-7647.

Hosking, S., Lough, A. J. \& Fekl, U. (2009). Acta Cryst. E65, m759$\mathrm{m} 760$.

Mohamed, A. A., Krause Bauer, J. A., Bruce, A. E. \& Bruce, M. R. M. (2003). Acta Cryst. C59, m84-m86.

Nguyen, N., Harrison, D. J., Lough, A. J., De Crisci, A. G. \& Fekl, U. (2010). Eur. J. Inorg. Chem. pp. 3577-3585.

Nonius (2002). COLLECT. Nonius BV, Delft, The Netherlands.

Otwinowski, Z. \& Minor, W. (1997). Methods in Enzymology, Vol. 276, Macromolecular Crystallography, Part A edited by C. W. Carter \& R. M. Sweet pp. 307-326. New York: Academic Press.

Parsons, S., Flack, H. D. \& Wagner, T. (2013). Acta Cryst. B69, 249-259. Sheldrick, G. M. (2008). Acta Cryst. A64, 112-122.

Sheldrick, G. M. (2015). Acta Cryst. C71, 3-8.

Spek, A. L. (2009). Acta Cryst. D65, 148-155.

Spek, A. L. (2015). Acta Cryst. C71, 9-18.

Stähler, R., Näther, C. \& Bensch, W. (2001). Acta Cryst. C57, 26-27. 


\section{supporting information}

Acta Cryst. (2017). E73, 1202-1207 [https://doi.org/10.1107/S205698901701026X]

\section{Coordination compounds containing bis-dithiolene-chelated molybdenum(IV) and oxalate: comparison of terminal with bridging oxalate}

\section{Agata Gapinska, Alan J. Lough and Ulrich Fekl}

\section{Computing details}

For both structures, data collection: COLLECT (Nonius, 2002); cell refinement: DENZO-SMN (Otwinowski \& Minor, 1997); data reduction: DENZO-SMN (Otwinowski \& Minor, 1997); program(s) used to solve structure: SIR92 (Altomare et al., 1994); program(s) used to refine structure: SHELXL2014 (Sheldrick, 2015); molecular graphics: ORTEP-3 (Farrugia, 2012) and PLATON (Spek, 2009); software used to prepare material for publication: SHELXTL (Sheldrick, 2008).

Bis(tetra- $n$-butylammonium) bis(1,1,1,4,4,4-hexafluorobut-2-ene-2,3-dithiolato)oxalatomolybdate(IV)chloroform-oxalic acid (1/1/1), (k10131)

\section{Crystal data}

$\left(\mathrm{C}_{16} \mathrm{H}_{36} \mathrm{~N}\right)_{2}\left[\mathrm{Mo}\left(\mathrm{C}_{4} \mathrm{~F}_{6} \mathrm{~S}_{2}\right)_{2}\left(\mathrm{C}_{2} \mathrm{O}_{4}\right)\right] \cdot \mathrm{C}_{2} \mathrm{H}_{2} \mathrm{O}_{4} \cdot \mathrm{CHCl}_{3}$

$M_{r}=1330.60$

Orthorhombic, $P 2{ }_{1} 2_{1} 2_{1}$

$a=15.3879$ (2) $\AA$

$b=17.8733(5) \AA$

$c=22.2895(6) \AA$

$V=6130.3(3) \AA^{3}$

$Z=4$

$F(000)=2752$

Data collection

Nonius KappaCCD diffractometer

Radiation source: fine-focus sealed tube Detector resolution: 9 pixels $\mathrm{mm}^{-1}$ $\varphi$ scans and $\omega$ scans with $\kappa$ offsets Absorption correction: multi-scan SORTAV (Blessing, 1995)

$T_{\min }=0.759, T_{\max }=0.869$

Refinement

Refinement on $F^{2}$

Least-squares matrix: full

$R\left[F^{2}>2 \sigma\left(F^{2}\right)\right]=0.048$

$w R\left(F^{2}\right)=0.105$

$S=1.02$

13841 reflections

816 parameters
$D_{\mathrm{x}}=1.442 \mathrm{Mg} \mathrm{m}^{-3}$

Mo $K \alpha$ radiation, $\lambda=0.71073 \AA$

Cell parameters from 31375 reflections

$\theta=2.6-27.5^{\circ}$

$\mu=0.56 \mathrm{~mm}^{-1}$

$T=150 \mathrm{~K}$

Block, brown

$0.15 \times 0.12 \times 0.10 \mathrm{~mm}$

40314 measured reflections 13841 independent reflections 9858 reflections with $I>2 \sigma(I)$

$R_{\text {int }}=0.065$

$\theta_{\max }=27.5^{\circ}, \theta_{\min }=2.6^{\circ}$

$h=-19 \rightarrow 19$

$k=-23 \rightarrow 23$

$l=-28 \rightarrow 28$

465 restraints

Hydrogen site location: mixed

$\mathrm{H}$ atoms treated by a mixture of independent and constrained refinement

$w=1 /\left[\sigma^{2}\left(F_{\mathrm{o}}^{2}\right)+(0.0407 P)^{2}+1.6697 P\right]$ where $P=\left(F_{\mathrm{o}}^{2}+2 F_{\mathrm{c}}^{2}\right) / 3$

$(\Delta / \sigma)_{\max }=0.001$ 


$$
\Delta \rho_{\max }=0.61 \text { e } \AA^{-3}
$$

$\Delta \rho_{\min }=-0.66$ e $\AA^{-3}$
Absolute structure: Flack $x$ determined using 3456 quotients $[(\mathrm{I}+)-(\mathrm{I}-)] /[(\mathrm{I}+)+(\mathrm{I}-)]$ (Parsons et al., 2013)

Absolute structure parameter: $-0.036(18)$

Special details

Geometry. All esds (except the esd in the dihedral angle between two 1.s. planes) are estimated using the full covariance matrix. The cell esds are taken into account individually in the estimation of esds in distances, angles and torsion angles; correlations between esds in cell parameters are only used when they are defined by crystal symmetry. An approximate (isotropic) treatment of cell esds is used for estimating esds involving l.s. planes.

Fractional atomic coordinates and isotropic or equivalent isotropic displacement parameters $\left(\AA^{2}\right)$

\begin{tabular}{|c|c|c|c|c|c|}
\hline & $x$ & $y$ & $z$ & $U_{\text {iso }} * / U_{\text {eq }}$ & Occ. $(<1)$ \\
\hline Mo1 & $0.53960(3)$ & $0.43946(2)$ & $0.51037(2)$ & $0.02584(12)$ & \\
\hline S1 & $0.66590(8)$ & $0.46380(8)$ & $0.45643(7)$ & $0.0323(3)$ & \\
\hline S2 & $0.52665(9)$ & $0.34333(8)$ & $0.44027(7)$ & 0.0337 (3) & \\
\hline S3 & $0.47571(9)$ & $0.35062(8)$ & $0.57355(7)$ & $0.0340(3)$ & \\
\hline S4 & $0.63308(8)$ & $0.45238(8)$ & $0.59206(6)$ & $0.0322(3)$ & \\
\hline F1 & $0.8097(2)$ & $0.3630(2)$ & $0.3621(2)$ & $0.0635(12)$ & \\
\hline $\mathrm{F} 2$ & $0.7261(3)$ & $0.4127(2)$ & $0.29583(17)$ & $0.0606(11)$ & \\
\hline F3 & $0.7876(2)$ & 0.4809 (2) & $0.36285(18)$ & $0.0591(11)$ & \\
\hline F4 & $0.6817(3)$ & $0.2702(2)$ & $0.31926(18)$ & $0.0577(10)$ & \\
\hline F5 & $0.5674(3)$ & $0.3269(2)$ & $0.28819(16)$ & $0.0606(11)$ & \\
\hline F6 & 0.5567 (2) & $0.23584(19)$ & $0.34988(16)$ & 0.0507 (9) & \\
\hline F7 & $0.4324(9)$ & $0.2510(8)$ & $0.6659(5)$ & $0.091(4)$ & $0.58(2)$ \\
\hline F8 & $0.5560(6)$ & $0.2515(6)$ & $0.7082(6)$ & $0.062(3)$ & $0.58(2)$ \\
\hline F9 & $0.4584(10)$ & $0.3280(6)$ & $0.7376(4)$ & $0.066(3)$ & $0.58(2)$ \\
\hline F7A & $0.4066(4)$ & $0.2880(9)$ & $0.6787(6)$ & $0.061(4)$ & $0.42(2)$ \\
\hline F8A & $0.5269(10)$ & $0.2300(5)$ & $0.6931(7)$ & $0.061(4)$ & $0.42(2)$ \\
\hline F9A & $0.4962(10)$ & $0.3283(8)$ & $0.7436(4)$ & 0.054 (4) & $0.42(2)$ \\
\hline F10 & $0.6162(7)$ & $0.3786(8)$ & $0.7546(4)$ & $0.083(4)$ & $0.502(10)$ \\
\hline F11 & $0.7244(7)$ & $0.3497(7)$ & $0.6994(5)$ & $0.079(4)$ & $0.502(10)$ \\
\hline F12 & $0.6920(8)$ & $0.4619(5)$ & $0.7150(5)$ & $0.061(3)$ & $0.502(10)$ \\
\hline F10A & $0.6267(8)$ & $0.4412(7)$ & $0.7472(4)$ & $0.084(4)$ & $0.498(10)$ \\
\hline F11A & 0.6637 (7) & $0.3297(5)$ & $0.7316(4)$ & 0.059 (3) & $0.498(10)$ \\
\hline F12A & $0.7358(7)$ & $0.4176(9)$ & $0.6927(5)$ & $0.084(4)$ & $0.498(10)$ \\
\hline $\mathrm{O} 1$ & $0.4106(2)$ & $0.46339(18)$ & $0.48554(18)$ & $0.0313(8)$ & \\
\hline $\mathrm{O} 2$ & $0.52764(19)$ & $0.55847(17)$ & $0.51231(16)$ & $0.0296(7)$ & \\
\hline $\mathrm{O} 3$ & $0.3096(2)$ & $0.5499(2)$ & 0.46934 (17) & $0.0339(9)$ & \\
\hline $\mathrm{O} 4$ & $0.4395(2)$ & $0.65521(19)$ & $0.4943(2)$ & $0.0452(11)$ & \\
\hline $\mathrm{C} 1$ & $0.6717(3)$ & $0.4035(3)$ & 0.3947 (3) & $0.0335(13)$ & \\
\hline $\mathrm{C} 2$ & $0.6103(3)$ & $0.3501(3)$ & 0.3889 (3) & $0.0313(13)$ & \\
\hline $\mathrm{C} 3$ & $0.7484(4)$ & 0.4144 (4) & $0.3540(3)$ & $0.0480(17)$ & \\
\hline $\mathrm{C} 4$ & 0.6037 (4) & 0.2963 (4) & $0.3374(3)$ & $0.0423(15)$ & \\
\hline $\mathrm{C} 5$ & $0.5288(4)$ & $0.3503(3)$ & $0.6422(3)$ & $0.0341(13)$ & \\
\hline C6 & $0.5997(3)$ & $0.3938(3)$ & $0.6504(3)$ & $0.0335(13)$ & \\
\hline $\mathrm{C} 7$ & $0.4924(3)$ & 0.2977 (3) & $0.6884(3)$ & $0.0517(17)$ & \\
\hline $\mathrm{C} 8$ & 0.6569 (4) & 0.3958 (4) & $0.7050(3)$ & $0.0452(15)$ & \\
\hline
\end{tabular}




\begin{tabular}{|c|c|c|c|c|}
\hline C9 & $0.3838(3)$ & 0.5309 & $0.4828(3)$ & $0.0282(12)$ \\
\hline $\mathrm{C} 10$ & $0.4552(3)$ & $0.5876(3)$ & $0.4974(2)$ & $0.0305(12)$ \\
\hline O5 & $0.6031(3)$ & $0.9245(2)$ & $0.4773(3)$ & $0.0739(17)$ \\
\hline O6 & $0.7088(3)$ & 0.8409 (2) & $0.4927(2)$ & $0.0486(12)$ \\
\hline $\mathrm{H} 6 \mathrm{O}$ & $0.748(4)$ & $0.874(4)$ & $0.504(4)$ & $0.07(2)^{*}$ \\
\hline $\mathrm{O} 7$ & $0.5404(3)$ & $0.7847(3)$ & $0.4176(2)$ & $0.0660(13)$ \\
\hline $\mathrm{O} 8$ & $0.5587(3)$ & $0.7544(2)$ & $0.5137(2)$ & $0.0496(12)$ \\
\hline $\mathrm{H} 8 \mathrm{O}$ & $0.521(5)$ & $0.720(4)$ & $0.509(4)$ & $0.09(3)^{*}$ \\
\hline C11 & $0.6297(4)$ & $0.8623(3)$ & $0.4796(3)$ & $0.0411(15)$ \\
\hline $\mathrm{C} 12$ & $0.5716(4)$ & $0.7956(3)$ & $0.4660(3)$ & $0.0392(15)$ \\
\hline N1 & $0.2406(3)$ & $0.1717(2)$ & $0.8798(2)$ & $0.0330(11)$ \\
\hline C13 & $0.1512(3)$ & $0.1525(3)$ & $0.8555(3)$ & $0.0403(15)$ \\
\hline H13A & 0.1077 & 0.1834 & 0.8769 & $0.048^{*}$ \\
\hline H13B & 0.1492 & 0.1671 & 0.8126 & $0.048 *$ \\
\hline C14 & $0.1240(3)$ & 0.0719 & $0.8603(3)$ & $0.0450(16)$ \\
\hline H14A & 0.1172 & 0.0585 & 0.9031 & $0.054^{*}$ \\
\hline H14B & 0.1701 & 0.0397 & 0.8431 & $0.054^{*}$ \\
\hline C15 & $0.0400(4)$ & $0.0572(4)$ & $0.8279(3)$ & $0.0608(18)$ \\
\hline $\mathrm{H} 15 \mathrm{~A}$ & -0.0041 & 0.0930 & 0.8428 & $0.073^{*}$ \\
\hline H15B & 0.0489 & 0.0677 & 0.7847 & $0.073^{*}$ \\
\hline C16 & $0.0044(5)$ & -0.0205 (4) & $0.8341(4)$ & $0.075(2)$ \\
\hline H16A & -0.0501 & -0.0245 & 0.8116 & $0.113 *$ \\
\hline H16B & 0.0465 & -0.0565 & 0.8183 & $0.113^{*}$ \\
\hline $\mathrm{H} 16 \mathrm{C}$ & -0.0066 & -0.0312 & 0.8766 & $0.113^{*}$ \\
\hline $\mathrm{C} 17$ & $0.3091(3)$ & 0.1358 (3) & $0.8406(3)$ & $0.0373(14)$ \\
\hline H17A & 0.3007 & 0.0809 & 0.8423 & $0.045^{*}$ \\
\hline H17B & 0.2982 & 0.1515 & 0.7986 & $0.045^{*}$ \\
\hline $\mathrm{C} 18$ & $0.4029(4)$ & $0.1520(3)$ & $0.8548(3)$ & $0.0434(15)$ \\
\hline H18A & 0.4169 & 0.1333 & 0.8955 & $0.052 *$ \\
\hline H18B & 0.4129 & 0.2067 & 0.8542 & $0.052 *$ \\
\hline C19 & $0.4612(4)$ & $0.1146(3)$ & $0.8095(3)$ & $0.0482(16)$ \\
\hline H19A & 0.4485 & 0.1356 & 0.7693 & $0.058 *$ \\
\hline H19B & 0.4472 & 0.0606 & 0.8083 & $0.058^{*}$ \\
\hline C20 & $0.5571(4)$ & $0.1237(5)$ & $0.8221(4)$ & $0.073(2)$ \\
\hline $\mathrm{H} 20 \mathrm{~A}$ & 0.5907 & 0.0981 & 0.7909 & $0.109^{*}$ \\
\hline $\mathrm{H} 20 \mathrm{~B}$ & 0.5720 & 0.1770 & 0.8223 & $0.109 *$ \\
\hline $\mathrm{H} 20 \mathrm{C}$ & 0.5707 & 0.1019 & 0.8613 & $0.109 *$ \\
\hline $\mathrm{C} 21$ & $0.2504(4)$ & $0.1446(3)$ & $0.9443(3)$ & $0.0390(14)$ \\
\hline $\mathrm{H} 21 \mathrm{~A}$ & 0.2486 & 0.0893 & 0.9441 & $0.047 *$ \\
\hline H21B & 0.3086 & 0.1597 & 0.9588 & $0.047^{*}$ \\
\hline $\mathrm{C} 22$ & $0.1836(4)$ & $0.1725(4)$ & $0.9886(3)$ & $0.0456(15)$ \\
\hline $\mathrm{H} 22 \mathrm{~A}$ & 0.1784 & 0.2275 & 0.9849 & $0.055^{*}$ \\
\hline $\mathrm{H} 22 \mathrm{~B}$ & 0.1264 & 0.1503 & 0.9788 & $0.055^{*}$ \\
\hline $\mathrm{C} 23$ & $0.2072(5)$ & $0.1529(5)$ & $1.0519(3)$ & $0.071(2)$ \\
\hline $\mathrm{H} 23 \mathrm{~A}$ & 0.2687 & 0.1668 & 1.0590 & $0.085^{*}$ \\
\hline $\mathrm{H} 23 \mathrm{~B}$ & 0.2021 & 0.0981 & 1.0572 & $0.085^{*}$ \\
\hline $\mathrm{C} 24$ & $0.1512(6)$ & $0.1912(5)$ & $1.0982(4)$ & $0.090(3)$ \\
\hline $\mathrm{H} 24 \mathrm{~A}$ & 0.1699 & 0.1761 & 1.1384 & $0.135^{*}$ \\
\hline
\end{tabular}




\begin{tabular}{|c|c|c|c|c|c|}
\hline H24B & 0.1570 & 0.2456 & 1.0940 & $0.135^{*}$ & \\
\hline $\mathrm{H} 24 \mathrm{C}$ & 0.0904 & 0.1768 & 1.0921 & $0.135^{*}$ & \\
\hline $\mathrm{C} 25$ & $0.2505(4)$ & $0.2567(3)$ & $0.8801(3)$ & $0.0383(15)$ & \\
\hline $\mathrm{H} 25 \mathrm{~A}$ & 0.2009 & 0.2779 & 0.9027 & $0.046^{*}$ & \\
\hline $\mathrm{H} 25 \mathrm{~B}$ & 0.3039 & 0.2690 & 0.9028 & $0.046^{*}$ & \\
\hline $\mathrm{C} 26$ & $0.2553(4)$ & 0.2964 & $0.8206(3)$ & $0.0393(14)$ & \\
\hline $\mathrm{H} 26 \mathrm{~A}$ & 0.2022 & 0.2856 & 0.7971 & $0.047^{*}$ & \\
\hline $\mathrm{H} 26 \mathrm{~B}$ & 0.3059 & 0.2778 & 0.7976 & $0.047 *$ & \\
\hline $\mathrm{C} 27$ & $0.2637(5)$ & $0.3796(3)$ & 0.8299 & $0.0517(18)$ & \\
\hline $\mathrm{H} 27 \mathrm{~A}$ & 0.2145 & 0.3970 & 0.8549 & $0.062 *$ & \\
\hline $\mathrm{H} 27 \mathrm{~B}$ & 0.3180 & 0.3897 & 0.8523 & $0.062 *$ & \\
\hline $\mathrm{C} 28$ & $0.2649(5)$ & $0.4242(3)$ & $0.7727(3)$ & $0.061(2)$ & \\
\hline $\mathrm{H} 28 \mathrm{~A}$ & 0.2704 & 0.4775 & 0.7823 & $0.092 *$ & \\
\hline $\mathrm{H} 28 \mathrm{~B}$ & 0.3142 & 0.4084 & 0.7480 & $0.092 *$ & \\
\hline $\mathrm{H} 28 \mathrm{C}$ & 0.2107 & 0.4157 & 0.7507 & $0.092 *$ & \\
\hline N2 & $0.7148(8)$ & $0.1623(6)$ & $0.5600(15)$ & $0.040(2)$ & $0.676(9)$ \\
\hline $\mathrm{C} 29$ & $0.6227(6)$ & $0.1461(5)$ & $0.5812(8)$ & $0.047(2)$ & $0.676(9)$ \\
\hline $\mathrm{H} 29 \mathrm{~A}$ & 0.6124 & 0.1738 & 0.6189 & $0.056^{*}$ & $0.676(9)$ \\
\hline H29B & 0.5815 & 0.1656 & 0.5509 & $0.056^{*}$ & $0.676(9)$ \\
\hline $\mathrm{C} 30$ & $0.6027(6)$ & $0.0649(6)$ & $0.5918(7)$ & $0.059(3)$ & $0.676(9)$ \\
\hline $\mathrm{H} 30 \mathrm{~A}$ & 0.6512 & 0.0424 & 0.6147 & $0.071^{*}$ & $0.676(9)$ \\
\hline H30B & 0.5996 & 0.0392 & 0.5525 & $0.071^{*}$ & $0.676(9)$ \\
\hline C31 & $0.5193(6)$ & $0.0509(6)$ & $0.6252(6)$ & $0.068(3)$ & $0.676(9)$ \\
\hline H31A & 0.4712 & 0.0771 & 0.6044 & $0.082 *$ & $0.676(9)$ \\
\hline H31B & 0.5241 & 0.0721 & 0.6661 & $0.082 *$ & $0.676(9)$ \\
\hline C32 & $0.4978(8)$ & $-0.0321(6)$ & $0.6296(7)$ & $0.093(4)$ & $0.676(9)$ \\
\hline $\mathrm{H} 32 \mathrm{~A}$ & 0.4431 & -0.0385 & 0.6517 & $0.139 *$ & $0.676(9)$ \\
\hline H32B & 0.4917 & -0.0531 & 0.5892 & $0.139 *$ & $0.676(9)$ \\
\hline $\mathrm{H} 32 \mathrm{C}$ & 0.5446 & -0.0581 & 0.6509 & $0.139 *$ & $0.676(9)$ \\
\hline C33 & $0.7243(6)$ & $0.2474(5)$ & $0.5539(6)$ & $0.052(2)$ & $0.676(9)$ \\
\hline H33A & 0.6747 & 0.2663 & 0.5300 & $0.062 *$ & $0.676(9)$ \\
\hline Н33В & 0.7197 & 0.2698 & 0.5944 & $0.062 *$ & $0.676(9)$ \\
\hline C34 & $0.8069(8)$ & $0.2748(5)$ & $0.5254(6)$ & $0.060(3)$ & $0.676(9)$ \\
\hline $\mathrm{H} 34 \mathrm{~A}$ & 0.8536 & 0.2380 & 0.5332 & $0.072 *$ & $0.676(9)$ \\
\hline H34B & 0.7984 & 0.2777 & 0.4814 & $0.072 *$ & $0.676(9)$ \\
\hline $\mathrm{C} 35$ & $0.8359(6)$ & $0.3499(6)$ & $0.5477(6)$ & $0.067(3)$ & $0.676(9)$ \\
\hline $\mathrm{H} 35 \mathrm{~A}$ & 0.8475 & 0.3466 & 0.5913 & $0.081 *$ & $0.676(9)$ \\
\hline H35B & 0.7883 & 0.3865 & 0.5417 & $0.081^{*}$ & $0.676(9)$ \\
\hline C36 & $0.9162(9)$ & $0.3777(9)$ & $0.5164(7)$ & $0.075(3)$ & $0.676(9)$ \\
\hline H36A & 0.9324 & 0.4267 & 0.5326 & $0.112 *$ & $0.676(9)$ \\
\hline H36B & 0.9639 & 0.3423 & 0.5230 & $0.112^{*}$ & $0.676(9)$ \\
\hline $\mathrm{H} 36 \mathrm{C}$ & 0.9047 & 0.3821 & 0.4733 & $0.112^{*}$ & $0.676(9)$ \\
\hline N2B & 0.7037 (17) & $0.1560(12)$ & $0.559(3)$ & $0.043(3)$ & $0.324(9)$ \\
\hline C29B & $0.6174(13)$ & $0.1228(10)$ & 0.5788 (19) & $0.047(4)$ & $0.324(9)$ \\
\hline $\mathrm{H} 29 \mathrm{C}$ & 0.5987 & 0.1487 & 0.6159 & $0.056^{*}$ & $0.324(9)$ \\
\hline H29D & 0.5737 & 0.1338 & 0.5475 & $0.056^{*}$ & $0.324(9)$ \\
\hline C30B & $0.6165(12)$ & $0.0401(10)$ & $0.5906(15)$ & 0.059 (4) & $0.324(9)$ \\
\hline $\mathrm{H} 30 \mathrm{C}$ & 0.6546 & 0.0290 & 0.6252 & $0.071^{*}$ & $0.324(9)$ \\
\hline
\end{tabular}




\begin{tabular}{|c|c|c|c|c|c|}
\hline H30D & 0.6402 & 0.0136 & 0.5552 & $0.071^{*}$ & $0.324(9)$ \\
\hline C31B & $0.5264(13)$ & $0.0112(13)$ & $0.6035(10)$ & $0.073(4)$ & $0.324(9)$ \\
\hline $\mathrm{H} 31 \mathrm{C}$ & 0.5213 & -0.0403 & 0.5875 & $0.088^{*}$ & $0.324(9)$ \\
\hline H31D & 0.4837 & 0.0428 & 0.5821 & $0.088^{*}$ & $0.324(9)$ \\
\hline C32B & $0.5037(18)$ & $0.010(2)$ & $0.6694(11)$ & $0.115(8)$ & $0.324(9)$ \\
\hline H32D & 0.4445 & -0.0090 & 0.6746 & $0.173^{*}$ & $0.324(9)$ \\
\hline $\mathrm{H} 32 \mathrm{E}$ & 0.5447 & -0.0220 & 0.6909 & $0.173^{*}$ & $0.324(9)$ \\
\hline $\mathrm{H} 32 \mathrm{~F}$ & 0.5071 & 0.0612 & 0.6855 & $0.173^{*}$ & $0.324(9)$ \\
\hline C33B & $0.6899(11)$ & $0.2402(9)$ & $0.5501(12)$ & $0.044(3)$ & $0.324(9)$ \\
\hline $\mathrm{H} 33 \mathrm{C}$ & 0.6355 & 0.2471 & 0.5269 & $0.053^{*}$ & $0.324(9)$ \\
\hline H33D & 0.6807 & 0.2632 & 0.5900 & $0.053^{*}$ & $0.324(9)$ \\
\hline C34B & 0.7607 (11) & $0.2829(10)$ & $0.5188(8)$ & $0.048(3)$ & $0.324(9)$ \\
\hline $\mathrm{H} 34 \mathrm{C}$ & 0.7356 & 0.3291 & 0.5015 & $0.058 *$ & $0.324(9)$ \\
\hline H34D & 0.7831 & 0.2522 & 0.4852 & $0.058^{*}$ & $0.324(9)$ \\
\hline C35B & $0.8356(10)$ & $0.3040(14)$ & $0.5587(9)$ & $0.053(4)$ & $0.324(9)$ \\
\hline $\mathrm{H} 35 \mathrm{C}$ & 0.8678 & 0.2582 & 0.5701 & $0.063 *$ & $0.324(9)$ \\
\hline H35D & 0.8127 & 0.3269 & 0.5960 & $0.063^{*}$ & $0.324(9)$ \\
\hline C36B & $0.897(2)$ & $0.358(2)$ & $0.5291(15)$ & $0.075(3)$ & $0.324(9)$ \\
\hline H36D & 0.9447 & 0.3700 & 0.5568 & $0.112 *$ & $0.324(9)$ \\
\hline H36E & 0.9211 & 0.3352 & 0.4927 & $0.112^{*}$ & $0.324(9)$ \\
\hline $\mathrm{H} 36 \mathrm{~F}$ & 0.8661 & 0.4039 & 0.5185 & $0.112 *$ & $0.324(9)$ \\
\hline C37 & $0.7786(4)$ & 0.1361 & 0.6058 & $0.0390(15)$ & \\
\hline H37A & 0.8371 & 0.1538 & 0.5938 & $0.047 *$ & \\
\hline H37B & 0.7798 & 0.0807 & 0.6051 & $0.047^{*}$ & \\
\hline C38 & $0.7617(4)$ & $0.1612(4)$ & $0.6693(3)$ & $0.0515(18)$ & \\
\hline H38A & 0.7524 & 0.2160 & 0.6698 & $0.062 *$ & \\
\hline H38B & 0.7080 & 0.1370 & 0.6842 & $0.062^{*}$ & \\
\hline C39 & $0.8358(4)$ & $0.1420(4)$ & $0.7105(3)$ & 0.059 (2) & \\
\hline H39A & 0.8887 & 0.1683 & 0.6966 & $0.070 *$ & \\
\hline Н39B & 0.8472 & 0.0876 & 0.7080 & $0.070^{*}$ & \\
\hline $\mathrm{C} 40$ & $0.8188(5)$ & $0.1628(5)$ & $0.7757(4)$ & $0.072(2)$ & \\
\hline $\mathrm{H} 40 \mathrm{~A}$ & 0.8693 & 0.1490 & 0.8001 & $0.107 *$ & \\
\hline H40B & 0.8089 & 0.2168 & 0.7787 & $0.107^{*}$ & \\
\hline $\mathrm{H} 40 \mathrm{C}$ & 0.7674 & 0.1360 & 0.7901 & $0.107 *$ & \\
\hline C41 & $0.7343(4)$ & 0.1213 & 0.5013 & $0.0406(15)$ & \\
\hline $\mathrm{H} 41 \mathrm{~A}$ & 0.7941 & 0.1346 & 0.4889 & $0.049 *$ & \\
\hline H41B & 0.7338 & 0.0669 & 0.5097 & $0.049 *$ & \\
\hline $\mathrm{C} 42$ & $0.6744(4)$ & $0.1355(4)$ & 0.4483 & $0.058(2)$ & \\
\hline $\mathrm{H} 42 \mathrm{~A}$ & 0.6192 & 0.1081 & 0.4544 & $0.069^{*}$ & \\
\hline $\mathrm{H} 42 \mathrm{~B}$ & 0.6607 & 0.1896 & 0.4461 & $0.069 *$ & \\
\hline $\mathrm{C} 43$ & $0.7156(5)$ & $0.1107(4)$ & $0.3897(3)$ & $0.0543(18)$ & \\
\hline $\mathrm{H} 43 \mathrm{~A}$ & 0.6733 & 0.1189 & 0.3569 & $0.065^{*}$ & \\
\hline H43B & 0.7665 & 0.1431 & 0.3816 & $0.065 *$ & \\
\hline C44 & $0.7451(5)$ & $0.0299(4)$ & $0.3876(3)$ & $0.062(2)$ & \\
\hline $\mathrm{H} 44 \mathrm{~A}$ & 0.7707 & 0.0192 & 0.3482 & $0.093^{*}$ & \\
\hline H44B & 0.7886 & 0.0212 & 0.4189 & $0.093 *$ & \\
\hline $\mathrm{H} 44 \mathrm{C}$ & 0.6952 & -0.0031 & 0.3942 & $0.093^{*}$ & \\
\hline Cl1 & $0.35534(12)$ & $0.10693(14)$ & $0.18122(13)$ & $0.0926(8)$ & \\
\hline
\end{tabular}




\begin{tabular}{lllll}
$\mathrm{C} 12$ & $0.51265(14)$ & $0.18119(11)$ & $0.21396(11)$ & $0.0774(6)$ \\
$\mathrm{Cl} 3$ & $0.51834(11)$ & $0.03822(10)$ & $0.15306(9)$ & $0.0622(5)$ \\
$\mathrm{C} 1 \mathrm{~S}$ & $0.4624(4)$ & $0.1240(4)$ & $0.1603(3)$ & $0.0525(16)$ \\
$\mathrm{H} 1 \mathrm{SA}$ & 0.4627 & 0.1504 & 0.1207 & $0.063^{*}$ \\
\hline
\end{tabular}

Atomic displacement parameters $\left(\AA^{2}\right)$

\begin{tabular}{|c|c|c|c|c|c|c|}
\hline & $U^{11}$ & $U^{22}$ & $U^{33}$ & $U^{12}$ & $U^{13}$ & $U^{23}$ \\
\hline Mo1 & $0.0273(2)$ & $0.0249(2)$ & $0.0253(2)$ & 0.00135 (19) & $0.00003(19)$ & $-0.0008(2)$ \\
\hline S1 & $0.0311(7)$ & $0.0373(8)$ & $0.0287(8)$ & $-0.0002(6)$ & $0.0041(6)$ & $-0.0022(7)$ \\
\hline $\mathrm{S} 2$ & $0.0380(8)$ & $0.0321(7)$ & $0.0308(8)$ & $-0.0003(6)$ & $0.0001(6)$ & $-0.0057(6)$ \\
\hline $\mathrm{S} 3$ & $0.0365(8)$ & $0.0338(8)$ & 0.0315 (9) & $-0.0040(6)$ & $0.0015(6)$ & $0.0032(6)$ \\
\hline S4 & $0.0373(7)$ & $0.0339(8)$ & $0.0254(8)$ & $-0.0024(6)$ & $-0.0040(6)$ & $0.0013(7)$ \\
\hline F1 & 0.049 (2) & $0.072(3)$ & 0.069 (3) & $0.0229(19)$ & $0.020(2)$ & $0.002(2)$ \\
\hline F2 & 0.077 (3) & $0.076(3)$ & $0.029(2)$ & $-0.002(2)$ & $0.0148(19)$ & $-0.001(2)$ \\
\hline F3 & $0.057(2)$ & 0.065 & $0.056(3)$ & $-0.0097(19)$ & $0.0256(19)$ & $-0.007(2)$ \\
\hline F4 & $0.073(3)$ & $0.051(2)$ & $0.049(3)$ & $0.0162(19)$ & $0.016(2)$ & $-0.013(2)$ \\
\hline F5 & 0.098 (3) & 0.058 (2) & $0.026(2)$ & 0.009 (2) & $-0.0160(19)$ & $-0.0038(19)$ \\
\hline F6 & $0.076(3)$ & $0.037(2)$ & $0.040(2)$ & $-0.0028(17)$ & $-0.0008(19)$ & $-0.0134(17)$ \\
\hline F7 & $0.112(8)$ & $0.087(8)$ & $0.075(7)$ & $-0.073(6)$ & $-0.024(6)$ & $0.044(6)$ \\
\hline F8 & $0.077(6)$ & $0.026(5)$ & $0.083(7)$ & -0.002 & $0.013(5)$ & $0.024(5)$ \\
\hline F9 & $0.068(8)$ & $0.068(5)$ & $0.063(6)$ & 0.009 (5) & $0.046(5)$ & $0.010(4)$ \\
\hline F7A & $0.068(6)$ & $0.052(8)$ & $0.063(7)$ & -0.007 (5) & 0.019 (5) & $0.013(6)$ \\
\hline F8A & $0.094(8)$ & $0.012(5)$ & $0.077(8)$ & $-0.008(5)$ & $0.041(7)$ & $0.008(5)$ \\
\hline F9A & $0.052(8)$ & $0.072(7)$ & $0.038(6)$ & $0.001(6)$ & $0.029(5)$ & $0.013(5)$ \\
\hline F10 & $0.111(7)$ & $0.113(9)$ & $0.026(5)$ & $-0.038(7)$ & $-0.005(5)$ & $0.015(6)$ \\
\hline F11 & $0.089(7)$ & $0.085(7)$ & $0.062(7)$ & $0.051(6)$ & $-0.043(6)$ & $-0.035(6)$ \\
\hline F12 & $0.102(8)$ & $0.036(5)$ & $0.045(6)$ & $-0.014(5)$ & $-0.035(5)$ & $0.004(4)$ \\
\hline F10A & $0.139(9)$ & $0.074(7)$ & $0.038(6)$ & $0.027(7)$ & $-0.028(6)$ & $-0.021(6)$ \\
\hline F11A & $0.077(6)$ & $0.058(6)$ & $0.043(6)$ & $0.014(5)$ & $-0.023(5)$ & $0.019(5)$ \\
\hline F12A & $0.075(6)$ & $0.127(10)$ & $0.049(6)$ & $-0.039(7)$ & $-0.027(5)$ & $0.033(7)$ \\
\hline $\mathrm{O} 1$ & $0.0303(18)$ & $0.0242(19)$ & $0.039(2)$ & $0.0030(14)$ & $-0.0042(17)$ & $-0.0043(18)$ \\
\hline $\mathrm{O} 2$ & $0.0271(17)$ & $0.0253(16)$ & $0.037(2)$ & $0.0000(15)$ & $-0.0035(16)$ & $-0.0018(19)$ \\
\hline $\mathrm{O} 3$ & $0.0251(18)$ & $0.033(2)$ & $0.043(2)$ & $0.0038(15)$ & $-0.0028(15)$ & 0.0037 (19) \\
\hline $\mathrm{O} 4$ & $0.037(2)$ & $0.025(2)$ & $0.074(3)$ & $0.0002(14)$ & $-0.007(2)$ & $0.003(2)$ \\
\hline $\mathrm{C} 1$ & 0.040 & 0.039 & $0.022(3)$ & $0.010(3)$ & $0.000(2)$ & $0.002(3)$ \\
\hline $\mathrm{C} 2$ & $0.033(3)$ & $0.031(3)$ & $0.029(3)$ & 0.009 (2) & $-0.004(2)$ & 0.001 (3) \\
\hline $\mathrm{C} 3$ & $0.046(4)$ & $0.059(5)$ & $0.039(4)$ & 0.008 & $0.015(3)$ & $-0.002(3)$ \\
\hline $\mathrm{C} 4$ & $0.056(4)$ & $0.040(4)$ & $0.031(4)$ & 0.008 & $0.004(3)$ & $-0.003(3)$ \\
\hline $\mathrm{C} 5$ & $0.042(3)$ & $0.034(3)$ & $0.027(3)$ & $0.006(3)$ & 0.005 & $0.000(3)$ \\
\hline C6 & 0.039 (3) & $0.035(3)$ & $0.026(3)$ & $0.008(3)$ & $-0.001(2)$ & -0.003 \\
\hline C7 & $0.066(4)$ & $0.051(4)$ & $0.039(4)$ & $-0.004(3)$ & 0.007 (3) & 0.009 (3) \\
\hline $\mathrm{C} 8$ & 0.059 (4) & $0.046(4)$ & $0.031(4)$ & $0.002(3)$ & $-0.002(3)$ & 0.005 \\
\hline C9 & $0.029(3)$ & $0.029(3)$ & $0.026(3)$ & $0.002(2)$ & $0.003(2)$ & $0.003(3)$ \\
\hline C10 & $0.032(3)$ & $0.028(3)$ & $0.031(3)$ & $0.002(2)$ & $0.005(2)$ & $-0.001(2)$ \\
\hline $\mathrm{O} 5$ & $0.053(3)$ & $0.037(3)$ & $0.131(5)$ & $0.001(2)$ & $-0.015(3)$ & 0.014 (3) \\
\hline O6 & $0.042(2)$ & $0.036(2)$ & $0.068(3)$ & $-0.0088(18)$ & $-0.013(2)$ & $0.006(2)$ \\
\hline $\mathrm{O} 7$ & $0.067(3)$ & $0.082(4)$ & 0.049 (3) & $-0.024(3)$ & $-0.017(3)$ & $0.006(3)$ \\
\hline
\end{tabular}




\begin{tabular}{|c|c|c|c|c|c|c|}
\hline $\mathrm{O} 8$ & $0.054(3)$ & $0.040(2)$ & $0.055(3)$ & -0.019 (2) & $-0.013(2)$ & $0.016(3)$ \\
\hline C11 & $0.040(3)$ & $0.042(4)$ & $0.041(4)$ & $-0.004(3)$ & $-0.005(3)$ & $0.012(3)$ \\
\hline $\mathrm{C} 12$ & $0.036(3)$ & $0.036(3)$ & $0.045(4)$ & $0.001(2)$ & $-0.004(3)$ & $0.005(3)$ \\
\hline N1 & $0.039(3)$ & $0.037(3)$ & $0.023(3)$ & $0.003(2)$ & $-0.003(2)$ & $0.004(2)$ \\
\hline $\mathrm{C} 13$ & $0.037(3)$ & $0.048(4)$ & $0.036(4)$ & $0.001(3)$ & $-0.004(3)$ & $0.000(3)$ \\
\hline $\mathrm{C} 14$ & $0.042(3)$ & $0.050(4)$ & $0.043(4)$ & $-0.005(3)$ & $-0.003(3)$ & $0.000(3)$ \\
\hline $\mathrm{C} 15$ & $0.060(4)$ & $0.066(4)$ & $0.057(5)$ & $-0.023(4)$ & $-0.010(4)$ & $0.004(4)$ \\
\hline $\mathrm{C} 16$ & $0.077(5)$ & $0.081(6)$ & $0.068(6)$ & $-0.031(4)$ & $-0.001(4)$ & $-0.006(5)$ \\
\hline $\mathrm{C} 17$ & $0.045(3)$ & $0.037(3)$ & $0.030(4)$ & $0.000(3)$ & $0.005(3)$ & $-0.001(3)$ \\
\hline $\mathrm{C} 18$ & $0.048(4)$ & $0.040(4)$ & $0.042(4)$ & $0.001(3)$ & $0.001(3)$ & 0.007 (3) \\
\hline C19 & $0.048(4)$ & $0.048(4)$ & 0.049 (4) & $-0.004(3)$ & $0.016(3)$ & 0.005 (3) \\
\hline $\mathrm{C} 20$ & $0.049(5)$ & $0.101(6)$ & $0.068(6)$ & 0.007 (4) & $0.008(4)$ & $0.005(5)$ \\
\hline $\mathrm{C} 21$ & $0.047(3)$ & $0.040(3)$ & $0.030(4)$ & $0.004(3)$ & $-0.002(3)$ & $0.000(3)$ \\
\hline $\mathrm{C} 22$ & $0.053(4)$ & $0.048(4)$ & $0.036(4)$ & $0.003(3)$ & $0.001(3)$ & $0.004(3)$ \\
\hline $\mathrm{C} 23$ & $0.086(5)$ & $0.085(6)$ & $0.041(5)$ & $0.037(5)$ & 0.017 (4) & $0.014(4)$ \\
\hline $\mathrm{C} 24$ & $0.107(7)$ & $0.123(8)$ & $0.040(5)$ & $0.051(6)$ & $0.012(5)$ & $0.002(5)$ \\
\hline $\mathrm{C} 25$ & $0.043(3)$ & $0.036(3)$ & $0.035(4)$ & $0.004(3)$ & $-0.006(3)$ & $0.000(3)$ \\
\hline $\mathrm{C} 26$ & $0.042(3)$ & $0.041(4)$ & $0.035(4)$ & $0.003(3)$ & $-0.003(3)$ & $0.003(3)$ \\
\hline $\mathrm{C} 27$ & 0.069 (4) & $0.037(4)$ & $0.049(5)$ & $-0.004(3)$ & $0.002(3)$ & $0.000(3)$ \\
\hline $\mathrm{C} 28$ & $0.084(5)$ & $0.041(4)$ & $0.059(5)$ & $0.006(3)$ & $0.003(4)$ & 0.009 (4) \\
\hline N2 & $0.034(4)$ & $0.044(4)$ & $0.042(4)$ & $0.014(3)$ & $0.009(5)$ & $0.005(4)$ \\
\hline $\mathrm{C} 29$ & $0.033(4)$ & $0.059(6)$ & $0.049(5)$ & $0.013(4)$ & 0.007 (4) & $0.000(6)$ \\
\hline $\mathrm{C} 30$ & $0.038(4)$ & $0.070(6)$ & $0.069(5)$ & $0.000(5)$ & $0.008(4)$ & $0.003(6)$ \\
\hline $\mathrm{C} 31$ & $0.044(5)$ & $0.085(7)$ & $0.075(7)$ & $0.005(5)$ & $0.012(5)$ & $0.017(6)$ \\
\hline C32 & $0.059(6)$ & $0.129(11)$ & $0.090(10)$ & $-0.025(7)$ & $0.021(7)$ & $0.011(8)$ \\
\hline $\mathrm{C} 33$ & $0.055(5)$ & $0.048(5)$ & $0.052(5)$ & 0.018 (4) & $0.014(5)$ & $0.001(4)$ \\
\hline C34 & $0.065(6)$ & $0.050(5)$ & $0.065(6)$ & $0.004(5)$ & $0.010(5)$ & $0.001(5)$ \\
\hline $\mathrm{C} 35$ & $0.051(5)$ & $0.070(6)$ & $0.081(7)$ & $0.003(5)$ & $0.005(5)$ & $-0.011(6)$ \\
\hline C36 & $0.060(7)$ & $0.078(9)$ & $0.087(8)$ & $-0.013(5)$ & $0.017(6)$ & $0.005(7)$ \\
\hline N2B & $0.039(6)$ & $0.045(6)$ & $0.046(6)$ & $0.015(6)$ & $0.008(6)$ & $0.004(6)$ \\
\hline C29B & $0.033(6)$ & $0.058(7)$ & $0.050(6)$ & $0.008(6)$ & 0.009 (6) & $0.004(7)$ \\
\hline $\mathrm{C} 30 \mathrm{~B}$ & $0.039(6)$ & $0.071(8)$ & $0.067(6)$ & $-0.002(6)$ & $0.003(6)$ & $0.009(7)$ \\
\hline C31B & $0.056(7)$ & $0.090(9)$ & $0.074(8)$ & $-0.006(8)$ & $0.011(7)$ & $0.005(8)$ \\
\hline C32B & $0.090(13)$ & $0.156(16)$ & 0.099 (15) & 0.004 (14) & $0.024(13)$ & 0.010 (14) \\
\hline C33B & $0.041(7)$ & $0.041(6)$ & $0.050(6)$ & $0.014(6)$ & $0.010(7)$ & $0.004(6)$ \\
\hline C34B & $0.043(7)$ & $0.043(6)$ & $0.060(7)$ & $0.014(6)$ & $0.011(7)$ & $0.000(6)$ \\
\hline C35B & $0.047(6)$ & $0.047(7)$ & $0.064(7)$ & $0.003(7)$ & $0.009(6)$ & $0.004(7)$ \\
\hline C36B & $0.060(7)$ & $0.078(9)$ & $0.087(8)$ & $-0.013(5)$ & $0.017(6)$ & $0.005(7)$ \\
\hline C37 & $0.034(3)$ & $0.041(4)$ & $0.042(4)$ & $0.006(3)$ & 0.007 (3) & $0.001(3)$ \\
\hline $\mathrm{C} 38$ & $0.050(4)$ & $0.054(4)$ & $0.051(5)$ & $0.008(3)$ & $0.003(3)$ & $-0.013(4)$ \\
\hline C39 & $0.044(4)$ & $0.077(5)$ & $0.055(5)$ & $0.004(3)$ & $-0.002(3)$ & -0.017 (4) \\
\hline $\mathrm{C} 40$ & $0.060(5)$ & $0.098(6)$ & $0.056(6)$ & $0.004(4)$ & -0.013 & $-0.014(5)$ \\
\hline $\mathrm{C} 41$ & $0.042(3)$ & $0.042(3)$ & $0.038(4)$ & $0.015(2)$ & 0.007 (3) & $-0.003(3)$ \\
\hline $\mathrm{C} 42$ & $0.051(4)$ & $0.071(5)$ & $0.052(5)$ & $0.020(3)$ & $0.005(3)$ & $0.008(4)$ \\
\hline $\mathrm{C} 43$ & $0.066(4)$ & $0.055(4)$ & $0.042(4)$ & $0.014(3)$ & $-0.006(3)$ & $0.000(4)$ \\
\hline $\mathrm{C} 44$ & $0.086(5)$ & $0.057(5)$ & $0.043(5)$ & $0.011(4)$ & $-0.005(4)$ & $0.000(4)$ \\
\hline Cl1 & $0.0468(11)$ & $0.1084(18)$ & $0.123(2)$ & $-0.0053(10)$ & $0.0036(11)$ & -0.0458 \\
\hline $\mathrm{Cl} 2$ & $0.0895(15)$ & $0.0661(13)$ & $0.0767(16)$ & $-0.0212(10)$ & $-0.0209(11)$ & $-0.0111(11)$ \\
\hline
\end{tabular}




\begin{tabular}{lllllll}
$\mathrm{C} 13$ & $0.0676(11)$ & $0.0700(12)$ & $0.0491(11)$ & $0.0132(9)$ & $0.0020(8)$ & $-0.0076(9)$ \\
$\mathrm{C} 1 \mathrm{~S}$ & $0.047(3)$ & $0.065(4)$ & $0.045(4)$ & $0.005(3)$ & $-0.005(4)$ & $0.000(3)$ \\
\hline
\end{tabular}

Geometric parameters $\left(A,{ }^{\circ}\right)$

\begin{tabular}{|c|c|c|c|}
\hline Mo1-O1 & $2.104(3)$ & $\mathrm{C} 26-\mathrm{H} 26 \mathrm{~B}$ & 0.9900 \\
\hline $\mathrm{Mo} 1-\mathrm{O} 2$ & $2.135(3)$ & $\mathrm{C} 27-\mathrm{C} 28$ & $1.501(8)$ \\
\hline Mo1-S1 & $2.3265(14)$ & $\mathrm{C} 27-\mathrm{H} 27 \mathrm{~A}$ & 0.9900 \\
\hline $\mathrm{Mo} 1-\mathrm{S} 2$ & $2.3309(15)$ & $\mathrm{C} 27-\mathrm{H} 27 \mathrm{~B}$ & 0.9900 \\
\hline Mo1-S4 & $2.3320(14)$ & $\mathrm{C} 28-\mathrm{H} 28 \mathrm{~A}$ & 0.9800 \\
\hline Mo1-S3 & $2.3390(15)$ & $\mathrm{C} 28-\mathrm{H} 28 \mathrm{~B}$ & 0.9800 \\
\hline $\mathrm{S} 1-\mathrm{C} 1$ & $1.750(6)$ & $\mathrm{C} 28-\mathrm{H} 28 \mathrm{C}$ & 0.9800 \\
\hline $\mathrm{S} 2-\mathrm{C} 2$ & $1.727(6)$ & $\mathrm{N} 2-\mathrm{C} 37$ & $1.49(3)$ \\
\hline $\mathrm{S} 3-\mathrm{C} 5$ & $1.734(6)$ & $\mathrm{N} 2-\mathrm{C} 29$ & $1.521(7)$ \\
\hline S4-C6 & $1.746(6)$ & $\mathrm{N} 2-\mathrm{C} 41$ & $1.53(3)$ \\
\hline $\mathrm{F} 1-\mathrm{C} 3$ & $1.330(7)$ & $\mathrm{N} 2-\mathrm{C} 33$ & $1.535(7)$ \\
\hline $\mathrm{F} 2-\mathrm{C} 3$ & $1.342(8)$ & $\mathrm{C} 29-\mathrm{C} 30$ & $1.501(8)$ \\
\hline F3- C3 & $1.347(7)$ & $\mathrm{C} 29-\mathrm{H} 29 \mathrm{~A}$ & 0.9900 \\
\hline $\mathrm{F} 4-\mathrm{C} 4$ & $1.350(7)$ & $\mathrm{C} 29-\mathrm{H} 29 \mathrm{~B}$ & 0.9900 \\
\hline $\mathrm{F} 5-\mathrm{C} 4$ & $1.346(7)$ & $\mathrm{C} 30-\mathrm{C} 31$ & $1.505(8)$ \\
\hline $\mathrm{F} 6-\mathrm{C} 4$ & $1.329(7)$ & $\mathrm{C} 30-\mathrm{H} 30 \mathrm{~A}$ & 0.9900 \\
\hline F7-C7 & $1.342(6)$ & $\mathrm{C} 30-\mathrm{H} 30 \mathrm{~B}$ & 0.9900 \\
\hline $\mathrm{F} 8-\mathrm{C} 7$ & $1.355(6)$ & $\mathrm{C} 31-\mathrm{C} 32$ & $1.524(9)$ \\
\hline F9-C7 & $1.330(6)$ & $\mathrm{C} 31-\mathrm{H} 31 \mathrm{~A}$ & 0.9900 \\
\hline $\mathrm{F} 7 \mathrm{~A}-\mathrm{C} 7$ & $1.349(6)$ & $\mathrm{C} 31-\mathrm{H} 31 \mathrm{~B}$ & 0.9900 \\
\hline $\mathrm{F} 8 \mathrm{~A}-\mathrm{C} 7$ & $1.326(6)$ & $\mathrm{C} 32-\mathrm{H} 32 \mathrm{~A}$ & 0.9800 \\
\hline $\mathrm{F} 9 \mathrm{~A}-\mathrm{C} 7$ & $1.349(6)$ & $\mathrm{C} 32-\mathrm{H} 32 \mathrm{~B}$ & 0.9800 \\
\hline $\mathrm{F} 10-\mathrm{C} 8$ & $1.306(11)$ & $\mathrm{C} 32-\mathrm{H} 32 \mathrm{C}$ & 0.9800 \\
\hline $\mathrm{F} 11-\mathrm{C} 8$ & $1.332(11)$ & C33-C34 & $1.504(8)$ \\
\hline $\mathrm{F} 12-\mathrm{C} 8$ & $1.318(10)$ & $\mathrm{C} 33-\mathrm{H} 33 \mathrm{~A}$ & 0.9900 \\
\hline $\mathrm{F} 10 \mathrm{~A}-\mathrm{C} 8$ & $1.326(11)$ & C $33-\mathrm{H} 33 \mathrm{~B}$ & 0.9900 \\
\hline $\mathrm{F} 11 \mathrm{~A}-\mathrm{C} 8$ & $1.325(10)$ & C34-C35 & $1.499(8)$ \\
\hline $\mathrm{F} 12 \mathrm{~A}-\mathrm{C} 8$ & $1.304(11)$ & $\mathrm{C} 34-\mathrm{H} 34 \mathrm{~A}$ & 0.9900 \\
\hline $\mathrm{O} 1-\mathrm{C} 9$ & $1.276(6)$ & C34-H34B & 0.9900 \\
\hline $\mathrm{O} 2-\mathrm{C} 10$ & $1.275(6)$ & $\mathrm{C} 35-\mathrm{C} 36$ & $1.504(9)$ \\
\hline $\mathrm{O} 3-\mathrm{C} 9$ & $1.229(6)$ & $\mathrm{C} 35-\mathrm{H} 35 \mathrm{~A}$ & 0.9900 \\
\hline $\mathrm{O} 4-\mathrm{C} 10$ & $1.235(6)$ & C $35-\mathrm{H} 35 \mathrm{~B}$ & 0.9900 \\
\hline $\mathrm{C} 1-\mathrm{C} 2$ & $1.349(8)$ & $\mathrm{C} 36-\mathrm{H} 36 \mathrm{~A}$ & 0.9800 \\
\hline $\mathrm{C} 1-\mathrm{C} 3$ & $1.500(8)$ & $\mathrm{C} 36-\mathrm{H} 36 \mathrm{~B}$ & 0.9800 \\
\hline $\mathrm{C} 2-\mathrm{C} 4$ & $1.502(8)$ & $\mathrm{C} 36-\mathrm{H} 36 \mathrm{C}$ & 0.9800 \\
\hline $\mathrm{C} 5-\mathrm{C} 6$ & $1.353(8)$ & $\mathrm{N} 2 \mathrm{~B}-\mathrm{C} 41$ & $1.51(7)$ \\
\hline $\mathrm{C} 5-\mathrm{C} 7$ & $1.502(8)$ & $\mathrm{N} 2 \mathrm{~B}-\mathrm{C} 29 \mathrm{~B}$ & $1.519(7)$ \\
\hline $\mathrm{C} 6-\mathrm{C} 8$ & $1.502(8)$ & $\mathrm{N} 2 \mathrm{~B}-\mathrm{C} 33 \mathrm{~B}$ & $1.533(8)$ \\
\hline $\mathrm{C} 9-\mathrm{C} 10$ & $1.528(7)$ & $\mathrm{N} 2 \mathrm{~B}-\mathrm{C} 37$ & $1.59(6)$ \\
\hline $\mathrm{O} 5-\mathrm{C} 11$ & $1.185(7)$ & $\mathrm{C} 29 \mathrm{~B}-\mathrm{C} 30 \mathrm{~B}$ & $1.502(9)$ \\
\hline $\mathrm{O} 6-\mathrm{C} 11$ & $1.310(7)$ & $\mathrm{C} 29 \mathrm{~B}-\mathrm{H} 29 \mathrm{C}$ & 0.9900 \\
\hline $\mathrm{O} 6-\mathrm{H} 6 \mathrm{O}$ & $0.88(7)$ & $\mathrm{C} 29 \mathrm{~B}-\mathrm{H} 29 \mathrm{D}$ & 0.9900 \\
\hline $\mathrm{O} 7-\mathrm{C} 12$ & $1.198(7)$ & $\mathrm{C} 30 \mathrm{~B}-\mathrm{C} 31 \mathrm{~B}$ & $1.507(9)$ \\
\hline
\end{tabular}




\begin{tabular}{|c|c|c|c|}
\hline $\mathrm{O} 8-\mathrm{C} 12$ & $1.308(8)$ & $\mathrm{C} 30 \mathrm{~B}-\mathrm{H} 30 \mathrm{C}$ & 0.9900 \\
\hline $\mathrm{O} 8-\mathrm{H} 8 \mathrm{O}$ & $0.85(8)$ & $\mathrm{C} 30 \mathrm{~B}-\mathrm{H} 30 \mathrm{D}$ & 0.9900 \\
\hline $\mathrm{C} 11-\mathrm{C} 12$ & $1.520(8)$ & $\mathrm{C} 31 \mathrm{~B}-\mathrm{C} 32 \mathrm{~B}$ & $1.510(10)$ \\
\hline $\mathrm{N} 1-\mathrm{C} 17$ & $1.513(7)$ & $\mathrm{C} 31 \mathrm{~B}-\mathrm{H} 31 \mathrm{C}$ & 0.9900 \\
\hline $\mathrm{N} 1-\mathrm{C} 13$ & $1.519(6)$ & $\mathrm{C} 31 \mathrm{~B}-\mathrm{H} 31 \mathrm{D}$ & 0.9900 \\
\hline $\mathrm{N} 1-\mathrm{C} 21$ & $1.524(8)$ & $\mathrm{C} 32 \mathrm{~B}-\mathrm{H} 32 \mathrm{D}$ & 0.9800 \\
\hline $\mathrm{N} 1-\mathrm{C} 25$ & $1.527(6)$ & $\mathrm{C} 32 \mathrm{~B}-\mathrm{H} 32 \mathrm{E}$ & 0.9800 \\
\hline $\mathrm{C} 13-\mathrm{C} 14$ & $1.503(7)$ & $\mathrm{C} 32 \mathrm{~B}-\mathrm{H} 32 \mathrm{~F}$ & 0.9800 \\
\hline $\mathrm{C} 13-\mathrm{H} 13 \mathrm{~A}$ & 0.9900 & $\mathrm{C} 33 \mathrm{~B}-\mathrm{C} 34 \mathrm{~B}$ & $1.502(9)$ \\
\hline $\mathrm{C} 13-\mathrm{H} 13 \mathrm{~B}$ & 0.9900 & $\mathrm{C} 33 \mathrm{~B}-\mathrm{H} 33 \mathrm{C}$ & 0.9900 \\
\hline $\mathrm{C} 14-\mathrm{C} 15$ & $1.504(7)$ & C33B-H33D & 0.9900 \\
\hline $\mathrm{C} 14-\mathrm{H} 14 \mathrm{~A}$ & 0.9900 & $\mathrm{C} 34 \mathrm{~B}-\mathrm{C} 35 \mathrm{~B}$ & $1.503(9)$ \\
\hline C14-H14B & 0.9900 & $\mathrm{C} 34 \mathrm{~B}-\mathrm{H} 34 \mathrm{C}$ & 0.9900 \\
\hline $\mathrm{C} 15-\mathrm{C} 16$ & $1.500(8)$ & $\mathrm{C} 34 \mathrm{~B}-\mathrm{H} 34 \mathrm{D}$ & 0.9900 \\
\hline C15-H15A & 0.9900 & $\mathrm{C} 35 \mathrm{~B}-\mathrm{C} 36 \mathrm{~B}$ & $1.507(10)$ \\
\hline C15-H15B & 0.9900 & $\mathrm{C} 35 \mathrm{~B}-\mathrm{H} 35 \mathrm{C}$ & 0.9900 \\
\hline $\mathrm{C} 16-\mathrm{H} 16 \mathrm{~A}$ & 0.9800 & $\mathrm{C} 35 \mathrm{~B}-\mathrm{H} 35 \mathrm{D}$ & 0.9900 \\
\hline $\mathrm{C} 16-\mathrm{H} 16 \mathrm{~B}$ & 0.9800 & $\mathrm{C} 36 \mathrm{~B}-\mathrm{H} 36 \mathrm{D}$ & 0.9800 \\
\hline $\mathrm{C} 16-\mathrm{H} 16 \mathrm{C}$ & 0.9800 & $\mathrm{C} 36 \mathrm{~B}-\mathrm{H} 36 \mathrm{E}$ & 0.9800 \\
\hline $\mathrm{C} 17-\mathrm{C} 18$ & $1.506(8)$ & $\mathrm{C} 36 \mathrm{~B}-\mathrm{H} 36 \mathrm{~F}$ & 0.9800 \\
\hline C17-H17A & 0.9900 & C37-C38 & $1.508(9)$ \\
\hline C17-H17B & 0.9900 & C37-H37A & 0.9900 \\
\hline $\mathrm{C} 18-\mathrm{C} 19$ & $1.506(8)$ & C37-H37B & 0.9900 \\
\hline $\mathrm{C} 18-\mathrm{H} 18 \mathrm{~A}$ & 0.9900 & C $38-\mathrm{C} 39$ & $1.504(9)$ \\
\hline C18-H18B & 0.9900 & $\mathrm{C} 38-\mathrm{H} 38 \mathrm{~A}$ & 0.9900 \\
\hline $\mathrm{C} 19-\mathrm{C} 20$ & $1.511(9)$ & C $38-\mathrm{H} 38 \mathrm{~B}$ & 0.9900 \\
\hline $\mathrm{C} 19-\mathrm{H} 19 \mathrm{~A}$ & 0.9900 & $\mathrm{C} 39-\mathrm{C} 40$ & $1.522(10)$ \\
\hline C19-H19B & 0.9900 & $\mathrm{C} 39-\mathrm{H} 39 \mathrm{~A}$ & 0.9900 \\
\hline $\mathrm{C} 20-\mathrm{H} 20 \mathrm{~A}$ & 0.9800 & C39-H39B & 0.9900 \\
\hline $\mathrm{C} 20-\mathrm{H} 20 \mathrm{~B}$ & 0.9800 & $\mathrm{C} 40-\mathrm{H} 40 \mathrm{~A}$ & 0.9800 \\
\hline $\mathrm{C} 20-\mathrm{H} 20 \mathrm{C}$ & 0.9800 & $\mathrm{C} 40-\mathrm{H} 40 \mathrm{~B}$ & 0.9800 \\
\hline $\mathrm{C} 21-\mathrm{C} 22$ & $1.511(8)$ & $\mathrm{C} 40-\mathrm{H} 40 \mathrm{C}$ & 0.9800 \\
\hline $\mathrm{C} 21-\mathrm{H} 21 \mathrm{~A}$ & 0.9900 & $\mathrm{C} 41-\mathrm{C} 42$ & $1.520(9)$ \\
\hline $\mathrm{C} 21-\mathrm{H} 21 \mathrm{~B}$ & 0.9900 & $\mathrm{C} 41-\mathrm{H} 41 \mathrm{~A}$ & 0.9900 \\
\hline $\mathrm{C} 22-\mathrm{C} 23$ & $1.499(10)$ & $\mathrm{C} 41-\mathrm{H} 41 \mathrm{~B}$ & 0.9900 \\
\hline $\mathrm{C} 22-\mathrm{H} 22 \mathrm{~A}$ & 0.9900 & $\mathrm{C} 42-\mathrm{C} 43$ & $1.519(9)$ \\
\hline $\mathrm{C} 22-\mathrm{H} 22 \mathrm{~B}$ & 0.9900 & $\mathrm{C} 42-\mathrm{H} 42 \mathrm{~A}$ & 0.9900 \\
\hline $\mathrm{C} 23-\mathrm{C} 24$ & $1.507(10)$ & $\mathrm{C} 42-\mathrm{H} 42 \mathrm{~B}$ & 0.9900 \\
\hline $\mathrm{C} 23-\mathrm{H} 23 \mathrm{~A}$ & 0.9900 & $\mathrm{C} 43-\mathrm{C} 44$ & $1.515(9)$ \\
\hline $\mathrm{C} 23-\mathrm{H} 23 \mathrm{~B}$ & 0.9900 & $\mathrm{C} 43-\mathrm{H} 43 \mathrm{~A}$ & 0.9900 \\
\hline $\mathrm{C} 24-\mathrm{H} 24 \mathrm{~A}$ & 0.9800 & $\mathrm{C} 43-\mathrm{H} 43 \mathrm{~B}$ & 0.9900 \\
\hline $\mathrm{C} 24-\mathrm{H} 24 \mathrm{~B}$ & 0.9800 & $\mathrm{C} 44-\mathrm{H} 44 \mathrm{~A}$ & 0.9800 \\
\hline $\mathrm{C} 24-\mathrm{H} 24 \mathrm{C}$ & 0.9800 & $\mathrm{C} 44-\mathrm{H} 44 \mathrm{~B}$ & 0.9800 \\
\hline $\mathrm{C} 25-\mathrm{C} 26$ & $1.506(7)$ & $\mathrm{C} 44-\mathrm{H} 44 \mathrm{C}$ & 0.9800 \\
\hline $\mathrm{C} 25-\mathrm{H} 25 \mathrm{~A}$ & 0.9900 & $\mathrm{C} 11-\mathrm{C} 1 \mathrm{~S}$ & $1.739(7)$ \\
\hline $\mathrm{C} 25-\mathrm{H} 25 \mathrm{~B}$ & 0.9900 & $\mathrm{Cl} 2-\mathrm{C} 1 \mathrm{~S}$ & $1.754(7)$ \\
\hline $\mathrm{C} 26-\mathrm{C} 27$ & $1.507(7)$ & $\mathrm{Cl} 3-\mathrm{C} 1 \mathrm{~S}$ & $1.767(7)$ \\
\hline $\mathrm{C} 26-\mathrm{H} 26 \mathrm{~A}$ & 0.9900 & $\mathrm{C} 1 \mathrm{~S}-\mathrm{H} 1 \mathrm{SA}$ & 1.0000 \\
\hline
\end{tabular}




\begin{tabular}{|c|c|c|c|}
\hline $\mathrm{O} 1-\mathrm{Mo} 1-\mathrm{O} 2$ & $73.83(12)$ & $\mathrm{C} 26-\mathrm{C} 27-\mathrm{H} 27 \mathrm{~A}$ & 108.7 \\
\hline $\mathrm{O} 1-\mathrm{Mo1}-\mathrm{S} 1$ & $127.88(12)$ & $\mathrm{C} 28-\mathrm{C} 27-\mathrm{H} 27 \mathrm{~B}$ & 108.7 \\
\hline $\mathrm{O} 2-\mathrm{Mo} 1-\mathrm{S} 1$ & $84.04(9)$ & $\mathrm{C} 26-\mathrm{C} 27-\mathrm{H} 27 \mathrm{~B}$ & 108.7 \\
\hline $\mathrm{O} 1-\mathrm{Mo} 1-\mathrm{S} 2$ & $83.86(10)$ & $\mathrm{H} 27 \mathrm{~A}-\mathrm{C} 27-\mathrm{H} 27 \mathrm{~B}$ & 107.6 \\
\hline $\mathrm{O} 2-\mathrm{Mo} 1-\mathrm{S} 2$ & $137.77(10)$ & $\mathrm{C} 27-\mathrm{C} 28-\mathrm{H} 28 \mathrm{~A}$ & 109.5 \\
\hline $\mathrm{S} 1-\mathrm{Mo} 1-\mathrm{S} 2$ & $82.12(5)$ & $\mathrm{C} 27-\mathrm{C} 28-\mathrm{H} 28 \mathrm{~B}$ & 109.5 \\
\hline $\mathrm{O} 1-\mathrm{Mo1}-\mathrm{S} 4$ & $140.08(11)$ & $\mathrm{H} 28 \mathrm{~A}-\mathrm{C} 28-\mathrm{H} 28 \mathrm{~B}$ & 109.5 \\
\hline $\mathrm{O} 2-\mathrm{Mo} 1-\mathrm{S} 4$ & $86.48(10)$ & $\mathrm{C} 27-\mathrm{C} 28-\mathrm{H} 28 \mathrm{C}$ & 109.5 \\
\hline $\mathrm{S} 1-\mathrm{Mo} 1-\mathrm{S} 4$ & $82.51(5)$ & $\mathrm{H} 28 \mathrm{~A}-\mathrm{C} 28-\mathrm{H} 28 \mathrm{C}$ & 109.5 \\
\hline $\mathrm{S} 2-\mathrm{Mo1}-\mathrm{S} 4$ & $130.47(5)$ & $\mathrm{H} 28 \mathrm{~B}-\mathrm{C} 28-\mathrm{H} 28 \mathrm{C}$ & 109.5 \\
\hline $\mathrm{O} 1-\mathrm{Mo1}-\mathrm{S} 3$ & $84.26(11)$ & $\mathrm{C} 37-\mathrm{N} 2-\mathrm{C} 29$ & $110.0(16)$ \\
\hline $\mathrm{O} 2-\mathrm{Mo} 1-\mathrm{S} 3$ & $128.89(10)$ & $\mathrm{C} 37-\mathrm{N} 2-\mathrm{C} 41$ & $107.8(7)$ \\
\hline $\mathrm{S} 1-\mathrm{Mo1} 1-\mathrm{S} 3$ & $142.11(5)$ & $\mathrm{C} 29-\mathrm{N} 2-\mathrm{C} 41$ & $110.9(16)$ \\
\hline $\mathrm{S} 2-\mathrm{Mo1}-\mathrm{S} 3$ & $82.37(5)$ & $\mathrm{C} 37-\mathrm{N} 2-\mathrm{C} 33$ & $108.0(16)$ \\
\hline $\mathrm{S} 4-\mathrm{Mo1}-\mathrm{S} 3$ & $81.74(5)$ & $\mathrm{C} 29-\mathrm{N} 2-\mathrm{C} 33$ & $107.8(6)$ \\
\hline $\mathrm{C} 1-\mathrm{S} 1-\mathrm{Mo} 1$ & $109.50(19)$ & $\mathrm{C} 41-\mathrm{N} 2-\mathrm{C} 33$ & $112.3(14)$ \\
\hline $\mathrm{C} 2-\mathrm{S} 2-\mathrm{Mo} 1$ & $109.2(2)$ & $\mathrm{C} 30-\mathrm{C} 29-\mathrm{N} 2$ & $115.1(6)$ \\
\hline $\mathrm{C} 5-\mathrm{S} 3-\mathrm{Mo} 1$ & $109.61(19)$ & $\mathrm{C} 30-\mathrm{C} 29-\mathrm{H} 29 \mathrm{~A}$ & 108.5 \\
\hline $\mathrm{C} 6-\mathrm{S} 4-\mathrm{Mo} 1$ & $109.9(2)$ & $\mathrm{N} 2-\mathrm{C} 29-\mathrm{H} 29 \mathrm{~A}$ & 108.5 \\
\hline $\mathrm{C} 9-\mathrm{O} 1-\mathrm{Mo} 1$ & $120.6(3)$ & $\mathrm{C} 30-\mathrm{C} 29-\mathrm{H} 29 \mathrm{~B}$ & 108.5 \\
\hline $\mathrm{C} 10-\mathrm{O} 2-\mathrm{Mo} 1$ & $118.5(3)$ & $\mathrm{N} 2-\mathrm{C} 29-\mathrm{H} 29 \mathrm{~B}$ & 108.5 \\
\hline $\mathrm{C} 2-\mathrm{C} 1-\mathrm{C} 3$ & $125.8(5)$ & $\mathrm{H} 29 \mathrm{~A}-\mathrm{C} 29-\mathrm{H} 29 \mathrm{~B}$ & 107.5 \\
\hline $\mathrm{C} 2-\mathrm{C} 1-\mathrm{S} 1$ & $118.4(4)$ & $\mathrm{C} 29-\mathrm{C} 30-\mathrm{C} 31$ & $114.4(7)$ \\
\hline $\mathrm{C} 3-\mathrm{C} 1-\mathrm{S} 1$ & $115.8(4)$ & $\mathrm{C} 29-\mathrm{C} 30-\mathrm{H} 30 \mathrm{~A}$ & 108.7 \\
\hline $\mathrm{C} 1-\mathrm{C} 2-\mathrm{C} 4$ & $125.0(5)$ & $\mathrm{C} 31-\mathrm{C} 30-\mathrm{H} 30 \mathrm{~A}$ & 108.7 \\
\hline $\mathrm{C} 1-\mathrm{C} 2-\mathrm{S} 2$ & $120.6(4)$ & $\mathrm{C} 29-\mathrm{C} 30-\mathrm{H} 30 \mathrm{~B}$ & 108.7 \\
\hline $\mathrm{C} 4-\mathrm{C} 2-\mathrm{S} 2$ & $114.3(4)$ & $\mathrm{C} 31-\mathrm{C} 30-\mathrm{H} 30 \mathrm{~B}$ & 108.7 \\
\hline $\mathrm{F} 1-\mathrm{C} 3-\mathrm{F} 2$ & $107.2(5)$ & $\mathrm{H} 30 \mathrm{~A}-\mathrm{C} 30-\mathrm{H} 30 \mathrm{~B}$ & 107.6 \\
\hline $\mathrm{F} 1-\mathrm{C} 3-\mathrm{F} 3$ & $105.8(5)$ & $\mathrm{C} 30-\mathrm{C} 31-\mathrm{C} 32$ & $112.3(8)$ \\
\hline $\mathrm{F} 2-\mathrm{C} 3-\mathrm{F} 3$ & $106.0(5)$ & $\mathrm{C} 30-\mathrm{C} 31-\mathrm{H} 31 \mathrm{~A}$ & 109.1 \\
\hline $\mathrm{F} 1-\mathrm{C} 3-\mathrm{C} 1$ & $112.7(5)$ & $\mathrm{C} 32-\mathrm{C} 31-\mathrm{H} 31 \mathrm{~A}$ & 109.1 \\
\hline $\mathrm{F} 2-\mathrm{C} 3-\mathrm{C} 1$ & $112.4(5)$ & $\mathrm{C} 30-\mathrm{C} 31-\mathrm{H} 31 \mathrm{~B}$ & 109.1 \\
\hline $\mathrm{F} 3-\mathrm{C} 3-\mathrm{C} 1$ & $112.3(5)$ & $\mathrm{C} 32-\mathrm{C} 31-\mathrm{H} 31 \mathrm{~B}$ & 109.1 \\
\hline $\mathrm{F} 6-\mathrm{C} 4-\mathrm{F} 5$ & $106.0(5)$ & $\mathrm{H} 31 \mathrm{~A}-\mathrm{C} 31-\mathrm{H} 31 \mathrm{~B}$ & 107.9 \\
\hline $\mathrm{F} 6-\mathrm{C} 4-\mathrm{F} 4$ & $105.4(5)$ & $\mathrm{C} 31-\mathrm{C} 32-\mathrm{H} 32 \mathrm{~A}$ & 109.5 \\
\hline $\mathrm{F} 5-\mathrm{C} 4-\mathrm{F} 4$ & $105.4(5)$ & $\mathrm{C} 31-\mathrm{C} 32-\mathrm{H} 32 \mathrm{~B}$ & 109.5 \\
\hline $\mathrm{F} 6-\mathrm{C} 4-\mathrm{C} 2$ & $113.4(5)$ & $\mathrm{H} 32 \mathrm{~A}-\mathrm{C} 32-\mathrm{H} 32 \mathrm{~B}$ & 109.5 \\
\hline $\mathrm{F} 5-\mathrm{C} 4-\mathrm{C} 2$ & $112.9(5)$ & $\mathrm{C} 31-\mathrm{C} 32-\mathrm{H} 32 \mathrm{C}$ & 109.5 \\
\hline $\mathrm{F} 4-\mathrm{C} 4-\mathrm{C} 2$ & $112.9(5)$ & $\mathrm{H} 32 \mathrm{~A}-\mathrm{C} 32-\mathrm{H} 32 \mathrm{C}$ & 109.5 \\
\hline $\mathrm{C} 6-\mathrm{C} 5-\mathrm{C} 7$ & $124.6(5)$ & $\mathrm{H} 32 \mathrm{~B}-\mathrm{C} 32-\mathrm{H} 32 \mathrm{C}$ & 109.5 \\
\hline $\mathrm{C} 6-\mathrm{C} 5-\mathrm{S} 3$ & $119.8(4)$ & $\mathrm{C} 34-\mathrm{C} 33-\mathrm{N} 2$ & $116.2(6)$ \\
\hline $\mathrm{C} 7-\mathrm{C} 5-\mathrm{S} 3$ & $115.6(4)$ & $\mathrm{C} 34-\mathrm{C} 33-\mathrm{H} 33 \mathrm{~A}$ & 108.2 \\
\hline $\mathrm{C} 5-\mathrm{C} 6-\mathrm{C} 8$ & $126.6(5)$ & $\mathrm{N} 2-\mathrm{C} 33-\mathrm{H} 33 \mathrm{~A}$ & 108.2 \\
\hline $\mathrm{C} 5-\mathrm{C} 6-\mathrm{S} 4$ & $118.7(4)$ & $\mathrm{C} 34-\mathrm{C} 33-\mathrm{H} 33 \mathrm{~B}$ & 108.2 \\
\hline $\mathrm{C} 8-\mathrm{C} 6-\mathrm{S} 4$ & $114.7(4)$ & N2-C33-H33B & 108.2 \\
\hline $\mathrm{F} 9-\mathrm{C} 7-\mathrm{F} 7$ & $106.8(5)$ & $\mathrm{H} 33 \mathrm{~A}-\mathrm{C} 33-\mathrm{H} 33 \mathrm{~B}$ & 107.4 \\
\hline $\mathrm{F} 8 \mathrm{~A}-\mathrm{C} 7-\mathrm{F} 9 \mathrm{~A}$ & $106.3(6)$ & $\mathrm{C} 35-\mathrm{C} 34-\mathrm{C} 33$ & $113.8(7)$ \\
\hline
\end{tabular}




\begin{tabular}{|c|c|}
\hline $\mathrm{F} 8 \mathrm{~A}-\mathrm{C} 7-\mathrm{F} 7 \mathrm{~A}$ & $106.7(5)$ \\
\hline $\mathrm{F} 9 \mathrm{~A}-\mathrm{C} 7-\mathrm{F} 7 \mathrm{~A}$ & $103.9(5)$ \\
\hline $\mathrm{F} 9-\mathrm{C} 7-\mathrm{F} 8$ & $105.3(5)$ \\
\hline $\mathrm{F} 7-\mathrm{C} 7-\mathrm{F} 8$ & $103.8(5)$ \\
\hline $\mathrm{F} 8 \mathrm{~A}-\mathrm{C} 7-\mathrm{C} 5$ & $118.5(7)$ \\
\hline $\mathrm{F} 9-\mathrm{C} 7-\mathrm{C} 5$ & $117.2(7)$ \\
\hline $\mathrm{F} 7-\mathrm{C} 7-\mathrm{C} 5$ & $112.9(6)$ \\
\hline $\mathrm{F} 9 \mathrm{~A}-\mathrm{C} 7-\mathrm{C} 5$ & $110.9(8)$ \\
\hline $\mathrm{F} 7 \mathrm{~A}-\mathrm{C} 7-\mathrm{C} 5$ & $109.6(7)$ \\
\hline $\mathrm{F} 8-\mathrm{C} 7-\mathrm{C} 5$ & $109.6(6)$ \\
\hline $\mathrm{F} 10-\mathrm{C} 8-\mathrm{F} 12$ & $105.3(9)$ \\
\hline $\mathrm{F} 12 \mathrm{~A}-\mathrm{C} 8-\mathrm{F} 11 \mathrm{~A}$ & $106.7(9)$ \\
\hline $\mathrm{F} 12 \mathrm{~A}-\mathrm{C} 8-\mathrm{F} 10 \mathrm{~A}$ & $107.0(10)$ \\
\hline $\mathrm{F} 11 \mathrm{~A}-\mathrm{C} 8-\mathrm{F} 10 \mathrm{~A}$ & $104.8(9)$ \\
\hline $\mathrm{F} 10-\mathrm{C} 8-\mathrm{F} 11$ & $107.9(10)$ \\
\hline $\mathrm{F} 12-\mathrm{C} 8-\mathrm{F} 11$ & $104.5(9)$ \\
\hline $\mathrm{F} 12 \mathrm{~A}-\mathrm{C} 8-\mathrm{C} 6$ & $112.4(7)$ \\
\hline $\mathrm{F} 10-\mathrm{C} 8-\mathrm{C} 6$ & $113.6(7)$ \\
\hline $\mathrm{F} 12-\mathrm{C} 8-\mathrm{C} 6$ & $113.4(6)$ \\
\hline $\mathrm{F} 11 \mathrm{~A}-\mathrm{C} 8-\mathrm{C} 6$ & $112.8(6)$ \\
\hline $\mathrm{F} 10 \mathrm{~A}-\mathrm{C} 8-\mathrm{C} 6$ & $112.5(6)$ \\
\hline $\mathrm{F} 11-\mathrm{C} 8-\mathrm{C} 6$ & $111.5(6)$ \\
\hline $\mathrm{O} 3-\mathrm{C} 9-\mathrm{O} 1$ & $124.9(5)$ \\
\hline $\mathrm{O} 3-\mathrm{C} 9-\mathrm{C} 10$ & $122.4(4)$ \\
\hline $\mathrm{O} 1-\mathrm{C} 9-\mathrm{C} 10$ & $112.6(4)$ \\
\hline $\mathrm{O} 4-\mathrm{C} 10-\mathrm{O} 2$ & $125.8(5)$ \\
\hline $\mathrm{O} 4-\mathrm{C} 10-\mathrm{C} 9$ & $119.8(4)$ \\
\hline $\mathrm{O} 2-\mathrm{C} 10-\mathrm{C} 9$ & $114.4(4)$ \\
\hline $\mathrm{C} 11-\mathrm{O} 6-\mathrm{H} 6 \mathrm{O}$ & $120(4)$ \\
\hline $\mathrm{C} 12-\mathrm{O} 8-\mathrm{H} 8 \mathrm{O}$ & $115(6)$ \\
\hline $\mathrm{O} 5-\mathrm{C} 11-\mathrm{O} 6$ & $127.2(6)$ \\
\hline $\mathrm{O} 5-\mathrm{C} 11-\mathrm{C} 12$ & $121.6(5)$ \\
\hline $\mathrm{O} 6-\mathrm{C} 11-\mathrm{C} 12$ & $111.2(5)$ \\
\hline $\mathrm{O} 7-\mathrm{C} 12-\mathrm{O} 8$ & $125.4(6)$ \\
\hline $\mathrm{O} 7-\mathrm{C} 12-\mathrm{C} 11$ & $122.9(6)$ \\
\hline $\mathrm{O} 8-\mathrm{C} 12-\mathrm{C} 11$ & $111.7(6)$ \\
\hline $\mathrm{C} 17-\mathrm{N} 1-\mathrm{C} 13$ & $109.2(4)$ \\
\hline $\mathrm{C} 17-\mathrm{N} 1-\mathrm{C} 21$ & $110.0(4)$ \\
\hline $\mathrm{C} 13-\mathrm{N} 1-\mathrm{C} 21$ & $110.8(4)$ \\
\hline $\mathrm{C} 17-\mathrm{N} 1-\mathrm{C} 25$ & $110.8(4)$ \\
\hline $\mathrm{C} 13-\mathrm{N} 1-\mathrm{C} 25$ & $108.5(4)$ \\
\hline $\mathrm{C} 21-\mathrm{N} 1-\mathrm{C} 25$ & $107.6(4)$ \\
\hline $\mathrm{C} 14-\mathrm{C} 13-\mathrm{N} 1$ & $116.3(4)$ \\
\hline $\mathrm{C} 14-\mathrm{C} 13-\mathrm{H} 13 \mathrm{~A}$ & 108.2 \\
\hline $\mathrm{N} 1-\mathrm{C} 13-\mathrm{H} 13 \mathrm{~A}$ & 108.2 \\
\hline $\mathrm{C} 14-\mathrm{C} 13-\mathrm{H} 13 \mathrm{~B}$ & 108.2 \\
\hline $\mathrm{N} 1-\mathrm{C} 13-\mathrm{H} 13 \mathrm{~B}$ & 108.2 \\
\hline $\mathrm{H} 13 \mathrm{~A}-\mathrm{C} 13-\mathrm{H} 13 \mathrm{~B}$ & 107.4 \\
\hline
\end{tabular}

\begin{tabular}{|c|c|}
\hline C $35-\mathrm{C} 34-\mathrm{H} 34 \mathrm{~A}$ & 108.8 \\
\hline $\mathrm{C} 33-\mathrm{C} 34-\mathrm{H} 34 \mathrm{~A}$ & 108.8 \\
\hline $\mathrm{C} 35-\mathrm{C} 34-\mathrm{H} 34 \mathrm{~B}$ & 108.8 \\
\hline $\mathrm{C} 33-\mathrm{C} 34-\mathrm{H} 34 \mathrm{~B}$ & 108.8 \\
\hline $\mathrm{H} 34 \mathrm{~A}-\mathrm{C} 34-\mathrm{H} 34 \mathrm{~B}$ & 107.7 \\
\hline $\mathrm{C} 34-\mathrm{C} 35-\mathrm{C} 36$ & $112.7(8)$ \\
\hline $\mathrm{C} 34-\mathrm{C} 35-\mathrm{H} 35 \mathrm{~A}$ & 109.1 \\
\hline $\mathrm{C} 36-\mathrm{C} 35-\mathrm{H} 35 \mathrm{~A}$ & 109.1 \\
\hline C $34-\mathrm{C} 35-\mathrm{H} 35 \mathrm{~B}$ & 109.1 \\
\hline $\mathrm{C} 36-\mathrm{C} 35-\mathrm{H} 35 \mathrm{~B}$ & 109.1 \\
\hline $\mathrm{H} 35 \mathrm{~A}-\mathrm{C} 35-\mathrm{H} 35 \mathrm{~B}$ & 107.8 \\
\hline $\mathrm{C} 35-\mathrm{C} 36-\mathrm{H} 36 \mathrm{~A}$ & 109.5 \\
\hline $\mathrm{C} 35-\mathrm{C} 36-\mathrm{H} 36 \mathrm{~B}$ & 109.5 \\
\hline $\mathrm{H} 36 \mathrm{~A}-\mathrm{C} 36-\mathrm{H} 36 \mathrm{~B}$ & 109.5 \\
\hline $\mathrm{C} 35-\mathrm{C} 36-\mathrm{H} 36 \mathrm{C}$ & 109.5 \\
\hline $\mathrm{H} 36 \mathrm{~A}-\mathrm{C} 36-\mathrm{H} 36 \mathrm{C}$ & 109.5 \\
\hline $\mathrm{H} 36 \mathrm{~B}-\mathrm{C} 36-\mathrm{H} 36 \mathrm{C}$ & 109.5 \\
\hline $\mathrm{C} 41-\mathrm{N} 2 \mathrm{~B}-\mathrm{C} 29 \mathrm{~B}$ & $111(4)$ \\
\hline $\mathrm{C} 41-\mathrm{N} 2 \mathrm{~B}-\mathrm{C} 33 \mathrm{~B}$ & $110(3)$ \\
\hline $\mathrm{C} 29 \mathrm{~B}-\mathrm{N} 2 \mathrm{~B}-\mathrm{C} 33 \mathrm{~B}$ & $107.5(8)$ \\
\hline $\mathrm{C} 41-\mathrm{N} 2 \mathrm{~B}-\mathrm{C} 37$ & $103.9(11)$ \\
\hline $\mathrm{C} 29 \mathrm{~B}-\mathrm{N} 2 \mathrm{~B}-\mathrm{C} 37$ & $111(3)$ \\
\hline $\mathrm{C} 33 \mathrm{~B}-\mathrm{N} 2 \mathrm{~B}-\mathrm{C} 37$ & $114(4)$ \\
\hline $\mathrm{C} 30 \mathrm{~B}-\mathrm{C} 29 \mathrm{~B}-\mathrm{N} 2 \mathrm{~B}$ & $116.3(9)$ \\
\hline $\mathrm{C} 30 \mathrm{~B}-\mathrm{C} 29 \mathrm{~B}-\mathrm{H} 29 \mathrm{C}$ & 108.2 \\
\hline $\mathrm{N} 2 \mathrm{~B}-\mathrm{C} 29 \mathrm{~B}-\mathrm{H} 29 \mathrm{C}$ & 108.2 \\
\hline $\mathrm{C} 30 \mathrm{~B}-\mathrm{C} 29 \mathrm{~B}-\mathrm{H} 29 \mathrm{D}$ & 108.2 \\
\hline $\mathrm{N} 2 \mathrm{~B}-\mathrm{C} 29 \mathrm{~B}-\mathrm{H} 29 \mathrm{D}$ & 108.2 \\
\hline $\mathrm{H} 29 \mathrm{C}-\mathrm{C} 29 \mathrm{~B}-\mathrm{H} 29 \mathrm{D}$ & 107.4 \\
\hline $\mathrm{C} 29 \mathrm{~B}-\mathrm{C} 30 \mathrm{~B}-\mathrm{C} 31 \mathrm{~B}$ & $112.3(10)$ \\
\hline $\mathrm{C} 29 \mathrm{~B}-\mathrm{C} 30 \mathrm{~B}-\mathrm{H} 30 \mathrm{C}$ & 109.1 \\
\hline $\mathrm{C} 31 \mathrm{~B}-\mathrm{C} 30 \mathrm{~B}-\mathrm{H} 30 \mathrm{C}$ & 109.1 \\
\hline $\mathrm{C} 29 \mathrm{~B}-\mathrm{C} 30 \mathrm{~B}-\mathrm{H} 30 \mathrm{D}$ & 109.1 \\
\hline $\mathrm{C} 31 \mathrm{~B}-\mathrm{C} 30 \mathrm{~B}-\mathrm{H} 30 \mathrm{D}$ & 109.1 \\
\hline $\mathrm{H} 30 \mathrm{C}-\mathrm{C} 30 \mathrm{~B}-\mathrm{H} 30 \mathrm{D}$ & 107.9 \\
\hline $\mathrm{C} 30 \mathrm{~B}-\mathrm{C} 31 \mathrm{~B}-\mathrm{C} 32 \mathrm{~B}$ & $113.7(10)$ \\
\hline $\mathrm{C} 30 \mathrm{~B}-\mathrm{C} 31 \mathrm{~B}-\mathrm{H} 31 \mathrm{C}$ & 108.8 \\
\hline $\mathrm{C} 32 \mathrm{~B}-\mathrm{C} 31 \mathrm{~B}-\mathrm{H} 31 \mathrm{C}$ & 108.8 \\
\hline $\mathrm{C} 30 \mathrm{~B}-\mathrm{C} 31 \mathrm{~B}-\mathrm{H} 31 \mathrm{D}$ & 108.8 \\
\hline $\mathrm{C} 32 \mathrm{~B}-\mathrm{C} 31 \mathrm{~B}-\mathrm{H} 31 \mathrm{D}$ & 108.8 \\
\hline $\mathrm{H} 31 \mathrm{C}-\mathrm{C} 31 \mathrm{~B}-\mathrm{H} 31 \mathrm{D}$ & 107.7 \\
\hline $\mathrm{C} 31 \mathrm{~B}-\mathrm{C} 32 \mathrm{~B}-\mathrm{H} 32 \mathrm{D}$ & 109.5 \\
\hline $\mathrm{C} 31 \mathrm{~B}-\mathrm{C} 32 \mathrm{~B}-\mathrm{H} 32 \mathrm{E}$ & 109.5 \\
\hline $\mathrm{H} 32 \mathrm{D}-\mathrm{C} 32 \mathrm{~B}-\mathrm{H} 32 \mathrm{E}$ & 109.5 \\
\hline $\mathrm{C} 31 \mathrm{~B}-\mathrm{C} 32 \mathrm{~B}-\mathrm{H} 32 \mathrm{~F}$ & 109.5 \\
\hline $\mathrm{H} 32 \mathrm{D}-\mathrm{C} 32 \mathrm{~B}-\mathrm{H} 32 \mathrm{~F}$ & 109.5 \\
\hline $\mathrm{H} 32 \mathrm{E}-\mathrm{C} 32 \mathrm{~B}-\mathrm{H} 32 \mathrm{~F}$ & 109.5 \\
\hline $\mathrm{C} 34 \mathrm{~B}-\mathrm{C} 33 \mathrm{~B}-\mathrm{N} 2 \mathrm{~B}$ & $117.3(9)$ \\
\hline
\end{tabular}




\begin{tabular}{|c|c|}
\hline $\mathrm{C} 13-\mathrm{C} 14-\mathrm{C} 15$ & $111.8(5)$ \\
\hline $\mathrm{C} 13-\mathrm{C} 14-\mathrm{H} 14 \mathrm{~A}$ & 109.3 \\
\hline $\mathrm{C} 15-\mathrm{C} 14-\mathrm{H} 14 \mathrm{~A}$ & 109.3 \\
\hline $\mathrm{C} 13-\mathrm{C} 14-\mathrm{H} 14 \mathrm{~B}$ & 109.3 \\
\hline $\mathrm{C} 15-\mathrm{C} 14-\mathrm{H} 14 \mathrm{~B}$ & 109.3 \\
\hline $\mathrm{H} 14 \mathrm{~A}-\mathrm{C} 14-\mathrm{H} 14 \mathrm{~B}$ & 107.9 \\
\hline $\mathrm{C} 16-\mathrm{C} 15-\mathrm{C} 14$ & $115.6(6)$ \\
\hline $\mathrm{C} 16-\mathrm{C} 15-\mathrm{H} 15 \mathrm{~A}$ & 108.4 \\
\hline $\mathrm{C} 14-\mathrm{C} 15-\mathrm{H} 15 \mathrm{~A}$ & 108.4 \\
\hline $\mathrm{C} 16-\mathrm{C} 15-\mathrm{H} 15 \mathrm{~B}$ & 108.4 \\
\hline $\mathrm{C} 14-\mathrm{C} 15-\mathrm{H} 15 \mathrm{~B}$ & 108.4 \\
\hline $\mathrm{H} 15 \mathrm{~A}-\mathrm{C} 15-\mathrm{H} 15 \mathrm{~B}$ & 107.5 \\
\hline $\mathrm{C} 15-\mathrm{C} 16-\mathrm{H} 16 \mathrm{~A}$ & 109.5 \\
\hline $\mathrm{C} 15-\mathrm{C} 16-\mathrm{H} 16 \mathrm{~B}$ & 109.5 \\
\hline $\mathrm{H} 16 \mathrm{~A}-\mathrm{C} 16-\mathrm{H} 16 \mathrm{~B}$ & 109.5 \\
\hline $\mathrm{C} 15-\mathrm{C} 16-\mathrm{H} 16 \mathrm{C}$ & 109.5 \\
\hline $\mathrm{H} 16 \mathrm{~A}-\mathrm{C} 16-\mathrm{H} 16 \mathrm{C}$ & 109.5 \\
\hline $\mathrm{H} 16 \mathrm{~B}-\mathrm{C} 16-\mathrm{H} 16 \mathrm{C}$ & 109.5 \\
\hline $\mathrm{C} 18-\mathrm{C} 17-\mathrm{N} 1$ & $117.7(5)$ \\
\hline $\mathrm{C} 18-\mathrm{C} 17-\mathrm{H} 17 \mathrm{~A}$ & 107.9 \\
\hline N1-C17-H17A & 107.9 \\
\hline $\mathrm{C} 18-\mathrm{C} 17-\mathrm{H} 17 \mathrm{~B}$ & 107.9 \\
\hline N1-C17-H17B & 107.9 \\
\hline H17A-C17-H17B & 107.2 \\
\hline $\mathrm{C} 17-\mathrm{C} 18-\mathrm{C} 19$ & $110.1(5)$ \\
\hline $\mathrm{C} 17-\mathrm{C} 18-\mathrm{H} 18 \mathrm{~A}$ & 109.6 \\
\hline $\mathrm{C} 19-\mathrm{C} 18-\mathrm{H} 18 \mathrm{~A}$ & 109.6 \\
\hline $\mathrm{C} 17-\mathrm{C} 18-\mathrm{H} 18 \mathrm{~B}$ & 109.6 \\
\hline $\mathrm{C} 19-\mathrm{C} 18-\mathrm{H} 18 \mathrm{~B}$ & 109.6 \\
\hline $\mathrm{H} 18 \mathrm{~A}-\mathrm{C} 18-\mathrm{H} 18 \mathrm{~B}$ & 108.2 \\
\hline $\mathrm{C} 18-\mathrm{C} 19-\mathrm{C} 20$ & $114.1(6)$ \\
\hline $\mathrm{C} 18-\mathrm{C} 19-\mathrm{H} 19 \mathrm{~A}$ & 108.7 \\
\hline $\mathrm{C} 20-\mathrm{C} 19-\mathrm{H} 19 \mathrm{~A}$ & 108.7 \\
\hline $\mathrm{C} 18-\mathrm{C} 19-\mathrm{H} 19 \mathrm{~B}$ & 108.7 \\
\hline $\mathrm{C} 20-\mathrm{C} 19-\mathrm{H} 19 \mathrm{~B}$ & 108.7 \\
\hline H19A-C19-H19B & 107.6 \\
\hline $\mathrm{C} 19-\mathrm{C} 20-\mathrm{H} 20 \mathrm{~A}$ & 109.5 \\
\hline $\mathrm{C} 19-\mathrm{C} 20-\mathrm{H} 20 \mathrm{~B}$ & 109.5 \\
\hline $\mathrm{H} 20 \mathrm{~A}-\mathrm{C} 20-\mathrm{H} 20 \mathrm{~B}$ & 109.5 \\
\hline $\mathrm{C} 19-\mathrm{C} 20-\mathrm{H} 20 \mathrm{C}$ & 109.5 \\
\hline $\mathrm{H} 20 \mathrm{~A}-\mathrm{C} 20-\mathrm{H} 20 \mathrm{C}$ & 109.5 \\
\hline $\mathrm{H} 20 \mathrm{~B}-\mathrm{C} 20-\mathrm{H} 20 \mathrm{C}$ & 109.5 \\
\hline $\mathrm{C} 22-\mathrm{C} 21-\mathrm{N} 1$ & $116.4(5)$ \\
\hline $\mathrm{C} 22-\mathrm{C} 21-\mathrm{H} 21 \mathrm{~A}$ & 108.2 \\
\hline $\mathrm{N} 1-\mathrm{C} 21-\mathrm{H} 21 \mathrm{~A}$ & 108.2 \\
\hline $\mathrm{C} 22-\mathrm{C} 21-\mathrm{H} 21 \mathrm{~B}$ & 108.2 \\
\hline $\mathrm{N} 1-\mathrm{C} 21-\mathrm{H} 21 \mathrm{~B}$ & 108.2 \\
\hline & \\
\hline
\end{tabular}

\begin{tabular}{|c|c|}
\hline $\mathrm{C} 34 \mathrm{~B}-\mathrm{C} 33 \mathrm{~B}-\mathrm{H} 33 \mathrm{C}$ & 108.0 \\
\hline $\mathrm{N} 2 \mathrm{~B}-\mathrm{C} 33 \mathrm{~B}-\mathrm{H} 33 \mathrm{C}$ & 108.0 \\
\hline $\mathrm{C} 34 \mathrm{~B}-\mathrm{C} 33 \mathrm{~B}-\mathrm{H} 33 \mathrm{D}$ & 108.0 \\
\hline $\mathrm{N} 2 \mathrm{~B}-\mathrm{C} 33 \mathrm{~B}-\mathrm{H} 33 \mathrm{D}$ & 108.0 \\
\hline $\mathrm{H} 33 \mathrm{C}-\mathrm{C} 33 \mathrm{~B}-\mathrm{H} 33 \mathrm{D}$ & 107.2 \\
\hline $\mathrm{C} 33 \mathrm{~B}-\mathrm{C} 34 \mathrm{~B}-\mathrm{C} 35 \mathrm{~B}$ & $114.2(10)$ \\
\hline $\mathrm{C} 33 \mathrm{~B}-\mathrm{C} 34 \mathrm{~B}-\mathrm{H} 34 \mathrm{C}$ & 108.7 \\
\hline $\mathrm{C} 35 \mathrm{~B}-\mathrm{C} 34 \mathrm{~B}-\mathrm{H} 34 \mathrm{C}$ & 108.7 \\
\hline $\mathrm{C} 33 \mathrm{~B}-\mathrm{C} 34 \mathrm{~B}-\mathrm{H} 34 \mathrm{D}$ & 108.7 \\
\hline $\mathrm{C} 35 \mathrm{~B}-\mathrm{C} 34 \mathrm{~B}-\mathrm{H} 34 \mathrm{D}$ & 108.7 \\
\hline $\mathrm{H} 34 \mathrm{C}-\mathrm{C} 34 \mathrm{~B}-\mathrm{H} 34 \mathrm{D}$ & 107.6 \\
\hline $\mathrm{C} 34 \mathrm{~B}-\mathrm{C} 35 \mathrm{~B}-\mathrm{C} 36 \mathrm{~B}$ & $112.6(10)$ \\
\hline $\mathrm{C} 34 \mathrm{~B}-\mathrm{C} 35 \mathrm{~B}-\mathrm{H} 35 \mathrm{C}$ & 109.1 \\
\hline $\mathrm{C} 36 \mathrm{~B}-\mathrm{C} 35 \mathrm{~B}-\mathrm{H} 35 \mathrm{C}$ & 109.1 \\
\hline $\mathrm{C} 34 \mathrm{~B}-\mathrm{C} 35 \mathrm{~B}-\mathrm{H} 35 \mathrm{D}$ & 109.1 \\
\hline $\mathrm{C} 36 \mathrm{~B}-\mathrm{C} 35 \mathrm{~B}-\mathrm{H} 35 \mathrm{D}$ & 109.1 \\
\hline $\mathrm{H} 35 \mathrm{C}-\mathrm{C} 35 \mathrm{~B}-\mathrm{H} 35 \mathrm{D}$ & 107.8 \\
\hline $\mathrm{C} 35 \mathrm{~B}-\mathrm{C} 36 \mathrm{~B}-\mathrm{H} 36 \mathrm{D}$ & 109.5 \\
\hline $\mathrm{C} 35 \mathrm{~B}-\mathrm{C} 36 \mathrm{~B}-\mathrm{H} 36 \mathrm{E}$ & 109.5 \\
\hline $\mathrm{H} 36 \mathrm{D}-\mathrm{C} 36 \mathrm{~B}-\mathrm{H} 36 \mathrm{E}$ & 109.5 \\
\hline $\mathrm{C} 35 \mathrm{~B}-\mathrm{C} 36 \mathrm{~B}-\mathrm{H} 36 \mathrm{~F}$ & 109.5 \\
\hline $\mathrm{H} 36 \mathrm{D}-\mathrm{C} 36 \mathrm{~B}-\mathrm{H} 36 \mathrm{~F}$ & 109.5 \\
\hline $\mathrm{H} 36 \mathrm{E}-\mathrm{C} 36 \mathrm{~B}-\mathrm{H} 36 \mathrm{~F}$ & 109.5 \\
\hline $\mathrm{N} 2-\mathrm{C} 37-\mathrm{C} 38$ & $115.8(10)$ \\
\hline $\mathrm{C} 38-\mathrm{C} 37-\mathrm{N} 2 \mathrm{~B}$ & $115(2)$ \\
\hline $\mathrm{N} 2-\mathrm{C} 37-\mathrm{H} 37 \mathrm{~A}$ & 108.3 \\
\hline $\mathrm{C} 38-\mathrm{C} 37-\mathrm{H} 37 \mathrm{~A}$ & 108.3 \\
\hline $\mathrm{N} 2-\mathrm{C} 37-\mathrm{H} 37 \mathrm{~B}$ & 108.3 \\
\hline C38-C37-H37B & 108.3 \\
\hline $\mathrm{H} 37 \mathrm{~A}-\mathrm{C} 37-\mathrm{H} 37 \mathrm{~B}$ & 107.4 \\
\hline $\mathrm{C} 39-\mathrm{C} 38-\mathrm{C} 37$ & $112.0(5)$ \\
\hline $\mathrm{C} 39-\mathrm{C} 38-\mathrm{H} 38 \mathrm{~A}$ & 109.2 \\
\hline $\mathrm{C} 37-\mathrm{C} 38-\mathrm{H} 38 \mathrm{~A}$ & 109.2 \\
\hline $\mathrm{C} 39-\mathrm{C} 38-\mathrm{H} 38 \mathrm{~B}$ & 109.2 \\
\hline $\mathrm{C} 37-\mathrm{C} 38-\mathrm{H} 38 \mathrm{~B}$ & 109.2 \\
\hline $\mathrm{H} 38 \mathrm{~A}-\mathrm{C} 38-\mathrm{H} 38 \mathrm{~B}$ & 107.9 \\
\hline $\mathrm{C} 38-\mathrm{C} 39-\mathrm{C} 40$ & $113.4(6)$ \\
\hline $\mathrm{C} 38-\mathrm{C} 39-\mathrm{H} 39 \mathrm{~A}$ & 108.9 \\
\hline $\mathrm{C} 40-\mathrm{C} 39-\mathrm{H} 39 \mathrm{~A}$ & 108.9 \\
\hline $\mathrm{C} 38-\mathrm{C} 39-\mathrm{H} 39 \mathrm{~B}$ & 108.9 \\
\hline $\mathrm{C} 40-\mathrm{C} 39-\mathrm{H} 39 \mathrm{~B}$ & 108.9 \\
\hline H39A-C39-H39B & 107.7 \\
\hline $\mathrm{C} 39-\mathrm{C} 40-\mathrm{H} 40 \mathrm{~A}$ & 109.5 \\
\hline $\mathrm{C} 39-\mathrm{C} 40-\mathrm{H} 40 \mathrm{~B}$ & 109.5 \\
\hline $\mathrm{H} 40 \mathrm{~A}-\mathrm{C} 40-\mathrm{H} 40 \mathrm{~B}$ & 109.5 \\
\hline $\mathrm{C} 39-\mathrm{C} 40-\mathrm{H} 40 \mathrm{C}$ & 109.5 \\
\hline $\mathrm{H} 40 \mathrm{~A}-\mathrm{C} 40-\mathrm{H} 40 \mathrm{C}$ & 109.5 \\
\hline $\mathrm{H} 40 \mathrm{~B}-\mathrm{C} 40-\mathrm{H} 40 \mathrm{C}$ & 109.5 \\
\hline
\end{tabular}




\begin{tabular}{|c|c|c|c|}
\hline $\mathrm{C} 23-\mathrm{C} 22-\mathrm{C} 21$ & $111.9(5)$ & $\mathrm{N} 2 \mathrm{~B}-\mathrm{C} 41-\mathrm{C} 42$ & $114.0(19)$ \\
\hline $\mathrm{C} 23-\mathrm{C} 22-\mathrm{H} 22 \mathrm{~A}$ & 109.2 & $\mathrm{C} 42-\mathrm{C} 41-\mathrm{N} 2$ & $117.8(9)$ \\
\hline $\mathrm{C} 21-\mathrm{C} 22-\mathrm{H} 22 \mathrm{~A}$ & 109.2 & $\mathrm{C} 42-\mathrm{C} 41-\mathrm{H} 41 \mathrm{~A}$ & 107.9 \\
\hline $\mathrm{C} 23-\mathrm{C} 22-\mathrm{H} 22 \mathrm{~B}$ & 109.2 & $\mathrm{~N} 2-\mathrm{C} 41-\mathrm{H} 41 \mathrm{~A}$ & 107.9 \\
\hline $\mathrm{C} 21-\mathrm{C} 22-\mathrm{H} 22 \mathrm{~B}$ & 109.2 & $\mathrm{C} 42-\mathrm{C} 41-\mathrm{H} 41 \mathrm{~B}$ & 107.9 \\
\hline $\mathrm{H} 22 \mathrm{~A}-\mathrm{C} 22-\mathrm{H} 22 \mathrm{~B}$ & 107.9 & $\mathrm{~N} 2-\mathrm{C} 41-\mathrm{H} 41 \mathrm{~B}$ & 107.9 \\
\hline $\mathrm{C} 22-\mathrm{C} 23-\mathrm{C} 24$ & $113.5(6)$ & $\mathrm{H} 41 \mathrm{~A}-\mathrm{C} 41-\mathrm{H} 41 \mathrm{~B}$ & 107.2 \\
\hline $\mathrm{C} 22-\mathrm{C} 23-\mathrm{H} 23 \mathrm{~A}$ & 108.9 & $\mathrm{C} 43-\mathrm{C} 42-\mathrm{C} 41$ & $111.5(5)$ \\
\hline $\mathrm{C} 24-\mathrm{C} 23-\mathrm{H} 23 \mathrm{~A}$ & 108.9 & $\mathrm{C} 43-\mathrm{C} 42-\mathrm{H} 42 \mathrm{~A}$ & 109.3 \\
\hline $\mathrm{C} 22-\mathrm{C} 23-\mathrm{H} 23 \mathrm{~B}$ & 108.9 & $\mathrm{C} 41-\mathrm{C} 42-\mathrm{H} 42 \mathrm{~A}$ & 109.3 \\
\hline $\mathrm{C} 24-\mathrm{C} 23-\mathrm{H} 23 \mathrm{~B}$ & 108.9 & $\mathrm{C} 43-\mathrm{C} 42-\mathrm{H} 42 \mathrm{~B}$ & 109.3 \\
\hline $\mathrm{H} 23 \mathrm{~A}-\mathrm{C} 23-\mathrm{H} 23 \mathrm{~B}$ & 107.7 & $\mathrm{C} 41-\mathrm{C} 42-\mathrm{H} 42 \mathrm{~B}$ & 109.3 \\
\hline $\mathrm{C} 23-\mathrm{C} 24-\mathrm{H} 24 \mathrm{~A}$ & 109.5 & $\mathrm{H} 42 \mathrm{~A}-\mathrm{C} 42-\mathrm{H} 42 \mathrm{~B}$ & 108.0 \\
\hline $\mathrm{C} 23-\mathrm{C} 24-\mathrm{H} 24 \mathrm{~B}$ & 109.5 & $\mathrm{C} 44-\mathrm{C} 43-\mathrm{C} 42$ & $115.5(6)$ \\
\hline $\mathrm{H} 24 \mathrm{~A}-\mathrm{C} 24-\mathrm{H} 24 \mathrm{~B}$ & 109.5 & $\mathrm{C} 44-\mathrm{C} 43-\mathrm{H} 43 \mathrm{~A}$ & 108.4 \\
\hline $\mathrm{C} 23-\mathrm{C} 24-\mathrm{H} 24 \mathrm{C}$ & 109.5 & $\mathrm{C} 42-\mathrm{C} 43-\mathrm{H} 43 \mathrm{~A}$ & 108.4 \\
\hline $\mathrm{H} 24 \mathrm{~A}-\mathrm{C} 24-\mathrm{H} 24 \mathrm{C}$ & 109.5 & $\mathrm{C} 44-\mathrm{C} 43-\mathrm{H} 43 \mathrm{~B}$ & 108.4 \\
\hline $\mathrm{H} 24 \mathrm{~B}-\mathrm{C} 24-\mathrm{H} 24 \mathrm{C}$ & 109.5 & $\mathrm{C} 42-\mathrm{C} 43-\mathrm{H} 43 \mathrm{~B}$ & 108.4 \\
\hline $\mathrm{C} 26-\mathrm{C} 25-\mathrm{N} 1$ & $118.0(4)$ & $\mathrm{H} 43 \mathrm{~A}-\mathrm{C} 43-\mathrm{H} 43 \mathrm{~B}$ & 107.5 \\
\hline $\mathrm{C} 26-\mathrm{C} 25-\mathrm{H} 25 \mathrm{~A}$ & 107.8 & $\mathrm{C} 43-\mathrm{C} 44-\mathrm{H} 44 \mathrm{~A}$ & 109.5 \\
\hline $\mathrm{N} 1-\mathrm{C} 25-\mathrm{H} 25 \mathrm{~A}$ & 107.8 & $\mathrm{C} 43-\mathrm{C} 44-\mathrm{H} 44 \mathrm{~B}$ & 109.5 \\
\hline $\mathrm{C} 26-\mathrm{C} 25-\mathrm{H} 25 \mathrm{~B}$ & 107.8 & $\mathrm{H} 44 \mathrm{~A}-\mathrm{C} 44-\mathrm{H} 44 \mathrm{~B}$ & 109.5 \\
\hline $\mathrm{N} 1-\mathrm{C} 25-\mathrm{H} 25 \mathrm{~B}$ & 107.8 & $\mathrm{C} 43-\mathrm{C} 44-\mathrm{H} 44 \mathrm{C}$ & 109.5 \\
\hline $\mathrm{H} 25 \mathrm{~A}-\mathrm{C} 25-\mathrm{H} 25 \mathrm{~B}$ & 107.1 & $\mathrm{H} 44 \mathrm{~A}-\mathrm{C} 44-\mathrm{H} 44 \mathrm{C}$ & 109.5 \\
\hline $\mathrm{C} 25-\mathrm{C} 26-\mathrm{C} 27$ & $110.4(5)$ & $\mathrm{H} 44 \mathrm{~B}-\mathrm{C} 44-\mathrm{H} 44 \mathrm{C}$ & 109.5 \\
\hline $\mathrm{C} 25-\mathrm{C} 26-\mathrm{H} 26 \mathrm{~A}$ & 109.6 & $\mathrm{Cl1}-\mathrm{C} 1 \mathrm{~S}-\mathrm{Cl} 2$ & $109.7(4)$ \\
\hline $\mathrm{C} 27-\mathrm{C} 26-\mathrm{H} 26 \mathrm{~A}$ & 109.6 & $\mathrm{C} 11-\mathrm{C} 1 \mathrm{~S}-\mathrm{Cl} 3$ & $109.5(4)$ \\
\hline $\mathrm{C} 25-\mathrm{C} 26-\mathrm{H} 26 \mathrm{~B}$ & 109.6 & $\mathrm{Cl} 2-\mathrm{C} 1 \mathrm{~S}-\mathrm{Cl} 3$ & $110.6(4)$ \\
\hline $\mathrm{C} 27-\mathrm{C} 26-\mathrm{H} 26 \mathrm{~B}$ & 109.6 & $\mathrm{C} 11-\mathrm{C} 1 \mathrm{~S}-\mathrm{H} 1 \mathrm{SA}$ & 109.0 \\
\hline $\mathrm{H} 26 \mathrm{~A}-\mathrm{C} 26-\mathrm{H} 26 \mathrm{~B}$ & 108.1 & $\mathrm{Cl} 2-\mathrm{C} 1 \mathrm{~S}-\mathrm{H} 1 \mathrm{SA}$ & 109.0 \\
\hline $\mathrm{C} 28-\mathrm{C} 27-\mathrm{C} 26$ & $114.1(5)$ & $\mathrm{Cl} 3-\mathrm{C} 1 \mathrm{~S}-\mathrm{H} 1 \mathrm{SA}$ & 109.0 \\
\hline $\mathrm{C} 28-\mathrm{C} 27-\mathrm{H} 27 \mathrm{~A}$ & 108.7 & & \\
\hline $\mathrm{Mo} 1-\mathrm{S} 1-\mathrm{C} 1-\mathrm{C} 2$ & $-4.8(5)$ & $\mathrm{O} 6-\mathrm{C} 11-\mathrm{C} 12-\mathrm{O} 7$ & $-114.9(7)$ \\
\hline $\mathrm{Mo} 1-\mathrm{S} 1-\mathrm{C} 1-\mathrm{C} 3$ & $177.8(4)$ & $\mathrm{O} 5-\mathrm{C} 11-\mathrm{C} 12-\mathrm{O} 8$ & $-113.7(7)$ \\
\hline $\mathrm{C} 3-\mathrm{C} 1-\mathrm{C} 2-\mathrm{C} 4$ & $-5.6(9)$ & $\mathrm{O} 6-\mathrm{C} 11-\mathrm{C} 12-\mathrm{O} 8$ & $67.0(7)$ \\
\hline $\mathrm{S} 1-\mathrm{C} 1-\mathrm{C} 2-\mathrm{C} 4$ & $177.2(4)$ & $\mathrm{C} 17-\mathrm{N} 1-\mathrm{C} 13-\mathrm{C} 14$ & $68.0(6)$ \\
\hline $\mathrm{C} 3-\mathrm{C} 1-\mathrm{C} 2-\mathrm{S} 2$ & $178.7(5)$ & $\mathrm{C} 21-\mathrm{N} 1-\mathrm{C} 13-\mathrm{C} 14$ & $-53.2(6)$ \\
\hline $\mathrm{S} 1-\mathrm{C} 1-\mathrm{C} 2-\mathrm{S} 2$ & $1.5(6)$ & $\mathrm{C} 25-\mathrm{N} 1-\mathrm{C} 13-\mathrm{C} 14$ & $-171.2(5)$ \\
\hline $\mathrm{Mo} 1-\mathrm{S} 2-\mathrm{C} 2-\mathrm{C} 1$ & $2.5(5)$ & $\mathrm{N} 1-\mathrm{C} 13-\mathrm{C} 14-\mathrm{C} 15$ & $-172.6(5)$ \\
\hline $\mathrm{Mo} 1-\mathrm{S} 2-\mathrm{C} 2-\mathrm{C} 4$ & $-173.7(4)$ & $\mathrm{C} 13-\mathrm{C} 14-\mathrm{C} 15-\mathrm{C} 16$ & $-175.6(6)$ \\
\hline $\mathrm{C} 2-\mathrm{C} 1-\mathrm{C} 3-\mathrm{F} 1$ & $-74.2(8)$ & $\mathrm{C} 13-\mathrm{N} 1-\mathrm{C} 17-\mathrm{C} 18$ & $176.3(5)$ \\
\hline $\mathrm{S} 1-\mathrm{C} 1-\mathrm{C} 3-\mathrm{F} 1$ & $103.1(6)$ & $\mathrm{C} 21-\mathrm{N} 1-\mathrm{C} 17-\mathrm{C} 18$ & $-62.0(6)$ \\
\hline $\mathrm{C} 2-\mathrm{C} 1-\mathrm{C} 3-\mathrm{F} 2$ & $47.1(8)$ & $\mathrm{C} 25-\mathrm{N} 1-\mathrm{C} 17-\mathrm{C} 18$ & $56.9(6)$ \\
\hline $\mathrm{S} 1-\mathrm{C} 1-\mathrm{C} 3-\mathrm{F} 2$ & $-135.6(5)$ & $\mathrm{N} 1-\mathrm{C} 17-\mathrm{C} 18-\mathrm{C} 19$ & $-177.3(5)$ \\
\hline $\mathrm{C} 2-\mathrm{C} 1-\mathrm{C} 3-\mathrm{F} 3$ & $166.5(6)$ & $\mathrm{C} 17-\mathrm{C} 18-\mathrm{C} 19-\mathrm{C} 20$ & $-176.4(6)$ \\
\hline $\mathrm{S} 1-\mathrm{C} 1-\mathrm{C} 3-\mathrm{F} 3$ & $-16.2(7)$ & $\mathrm{C} 17-\mathrm{N} 1-\mathrm{C} 21-\mathrm{C} 22$ & $-177.0(5)$ \\
\hline $\mathrm{C} 1-\mathrm{C} 2-\mathrm{C} 4-\mathrm{F} 6$ & $160.3(5)$ & $\mathrm{C} 13-\mathrm{N} 1-\mathrm{C} 21-\mathrm{C} 22$ & $-56.2(6)$ \\
\hline
\end{tabular}




$\begin{array}{ll}\text { S2-C2-C4-F6 } & -23.8(6) \\ \text { C1-C2-C4-F5 } & -79.1(7) \\ \text { S2-C2-C4-F5 } & 96.8(5) \\ \text { C1-C2-C4-F4 } & 40.4(8) \\ \text { S2-C2-C4-F4 } & -143.7(4) \\ \text { Mo1-S3-C5-C6 } & -4.3(5) \\ \text { Mo1-S3-C5-C7 } & 177.7(3) \\ \text { C7-C5-C6-C8 } & 2.7(9) \\ \text { S3-C5-C6-C8 } & -175.0(5) \\ \text { C7-C5-C6-S4 } & -179.6(4) \\ \text { S3-C5-C6-S4 } & 2.6(6) \\ \text { Mo1-S4-C6-C5 } & 0.4(5) \\ \text { Mo1-S4-C6-C8 } & 178.3(4) \\ \text { C6-C5-C7-F8A } & -83.8(11) \\ \text { S3-C5-C7-F8A } & 94.1(10) \\ \text { C6-C5-C7-F9 } & 66.9(10) \\ \text { S3-C5-C7-F9 } & -115.3(8) \\ \text { C6-C5-C7-F7 } & -168.3(10) \\ \text { S3-C5-C7-F7 } & 9.6(10) \\ \text { C6-C5-C7-F9A } & 39.4(10) \\ \text { S3-C5-C7-F9A } & -142.7(8) \\ \text { C6-C5-C7-F7A } & 153.6(9) \\ \text { S3-C5-C7-F7A } & -28.6(8) \\ \text { C6-C5-C7-F8 } & -53.0(9) \\ \text { S3-C5-C7-F8 } & 124.8(8) \\ \text { C5-C6-C8-F12A } & 154.0(10) \\ \text { S4-C6-C8-F12A } & -23.8(11) \\ \text { C5-C6-C8-F10 } & -28.9(12) \\ \text { S4-C6-C8-F10 } & 153.4(9) \\ \text { C5-C6-C8-F12 } & -149.1(9) \\ \text { S4-C6-C8-F12 } & 33.2(10) \\ \text { C5-C6-C8-F11A } & 33.3(10) \\ \text { S4-C6-C8-F11A } & -144.5(7) \\ \text { C5-C6-C8-F10A } & -85.1(11) \\ \text { S4-C6-C8-F10A } & 97.1(9) \\ \text { C5-C6-C8-F11 } & 93.3(10) \\ \text { S4-C6-C8-F11 } & -84.4(10) \\ \text { Mo1-O1-C9-O3 } & -179.3(4) \\ \text { Mo1-O1-C9-C10 } & 1.6(6) \\ \text { Mo1-O2-C10-O4 } & -177.2(4) \\ \text { Mo1-O2-C10-C9 } & 2.9(6) \\ \text { O3-C9-C10-O4 } & -2.0(8) \\ \text { O1-C9-C10-O4 } & 177.1(5) \\ \text { O3-C9-C10-O2 } & \\ \text { O1-C9-C10-O2 } & \\ \text { O5-C11-C12-O7 } & \\ & \end{array}$

\begin{tabular}{|c|c|}
\hline $\mathrm{C} 25-\mathrm{N} 1-\mathrm{C} 21-\mathrm{C} 22$ & $62.3(6)$ \\
\hline $\mathrm{N} 1-\mathrm{C} 21-\mathrm{C} 22-\mathrm{C} 23$ & $-170.7(6)$ \\
\hline $\mathrm{C} 21-\mathrm{C} 22-\mathrm{C} 23-\mathrm{C} 24$ & $169.7(7)$ \\
\hline $\mathrm{C} 17-\mathrm{N} 1-\mathrm{C} 25-\mathrm{C} 26$ & $51.8(6)$ \\
\hline $\mathrm{C} 13-\mathrm{N} 1-\mathrm{C} 25-\mathrm{C} 26$ & $-68.0(6)$ \\
\hline $\mathrm{C} 21-\mathrm{N} 1-\mathrm{C} 25-\mathrm{C} 26$ & $172.1(5)$ \\
\hline $\mathrm{N} 1-\mathrm{C} 25-\mathrm{C} 26-\mathrm{C} 27$ & $179.2(5)$ \\
\hline $\mathrm{C} 25-\mathrm{C} 26-\mathrm{C} 27-\mathrm{C} 28$ & $-177.4(6)$ \\
\hline $\mathrm{C} 37-\mathrm{N} 2-\mathrm{C} 29-\mathrm{C} 30$ & $-60(2)$ \\
\hline $\mathrm{C} 41-\mathrm{N} 2-\mathrm{C} 29-\mathrm{C} 30$ & $58.8(19)$ \\
\hline $\mathrm{C} 33-\mathrm{N} 2-\mathrm{C} 29-\mathrm{C} 30$ & $-177.9(17)$ \\
\hline $\mathrm{N} 2-\mathrm{C} 29-\mathrm{C} 30-\mathrm{C} 31$ & $167.2(18)$ \\
\hline $\mathrm{C} 29-\mathrm{C} 30-\mathrm{C} 31-\mathrm{C} 32$ & $174.7(13)$ \\
\hline $\mathrm{C} 37-\mathrm{N} 2-\mathrm{C} 33-\mathrm{C} 34$ & $69.2(18)$ \\
\hline $\mathrm{C} 29-\mathrm{N} 2-\mathrm{C} 33-\mathrm{C} 34$ & $-172.0(16)$ \\
\hline $\mathrm{C} 41-\mathrm{N} 2-\mathrm{C} 33-\mathrm{C} 34$ & $-49.5(18)$ \\
\hline $\mathrm{N} 2-\mathrm{C} 33-\mathrm{C} 34-\mathrm{C} 35$ & $-149.6(17)$ \\
\hline $\mathrm{C} 33-\mathrm{C} 34-\mathrm{C} 35-\mathrm{C} 36$ & $-177.2(11)$ \\
\hline $\mathrm{C} 41-\mathrm{N} 2 \mathrm{~B}-\mathrm{C} 29 \mathrm{~B}-\mathrm{C} 30 \mathrm{~B}$ & $60(4)$ \\
\hline $\mathrm{C} 33 \mathrm{~B}-\mathrm{N} 2 \mathrm{~B}-\mathrm{C} 29 \mathrm{~B}-\mathrm{C} 30 \mathrm{~B}$ & $-180(4)$ \\
\hline $\mathrm{C} 37-\mathrm{N} 2 \mathrm{~B}-\mathrm{C} 29 \mathrm{~B}-\mathrm{C} 30 \mathrm{~B}$ & $-55(5)$ \\
\hline $\mathrm{N} 2 \mathrm{~B}-\mathrm{C} 29 \mathrm{~B}-\mathrm{C} 30 \mathrm{~B}-\mathrm{C} 31 \mathrm{~B}$ & $-174(4)$ \\
\hline $\mathrm{C} 29 \mathrm{~B}-\mathrm{C} 30 \mathrm{~B}-\mathrm{C} 31 \mathrm{~B}-\mathrm{C} 32 \mathrm{~B}$ & $-92(3)$ \\
\hline $\mathrm{C} 41-\mathrm{N} 2 \mathrm{~B}-\mathrm{C} 33 \mathrm{~B}-\mathrm{C} 34 \mathrm{~B}$ & $-47(4)$ \\
\hline $\mathrm{C} 29 \mathrm{~B}-\mathrm{N} 2 \mathrm{~B}-\mathrm{C} 33 \mathrm{~B}-\mathrm{C} 34 \mathrm{~B}$ & $-168(3)$ \\
\hline $\mathrm{C} 37-\mathrm{N} 2 \mathrm{~B}-\mathrm{C} 33 \mathrm{~B}-\mathrm{C} 34 \mathrm{~B}$ & $69(4)$ \\
\hline $\mathrm{N} 2 \mathrm{~B}-\mathrm{C} 33 \mathrm{~B}-\mathrm{C} 34 \mathrm{~B}-\mathrm{C} 35 \mathrm{~B}$ & $-81(3)$ \\
\hline $\mathrm{C} 33 \mathrm{~B}-\mathrm{C} 34 \mathrm{~B}-\mathrm{C} 35 \mathrm{~B}-\mathrm{C} 36 \mathrm{~B}$ & $-170(2)$ \\
\hline $\mathrm{C} 29-\mathrm{N} 2-\mathrm{C} 37-\mathrm{C} 38$ & $-50.0(13)$ \\
\hline $\mathrm{C} 41-\mathrm{N} 2-\mathrm{C} 37-\mathrm{C} 38$ & $-171.0(5)$ \\
\hline $\mathrm{C} 33-\mathrm{N} 2-\mathrm{C} 37-\mathrm{C} 38$ & $67.4(11)$ \\
\hline $\mathrm{C} 41-\mathrm{N} 2 \mathrm{~B}-\mathrm{C} 37-\mathrm{C} 38$ & $-176.8(7)$ \\
\hline $\mathrm{C} 29 \mathrm{~B}-\mathrm{N} 2 \mathrm{~B}-\mathrm{C} 37-\mathrm{C} 38$ & $-57(3)$ \\
\hline $\mathrm{C} 33 \mathrm{~B}-\mathrm{N} 2 \mathrm{~B}-\mathrm{C} 37-\mathrm{C} 38$ & $64(2)$ \\
\hline $\mathrm{N} 2-\mathrm{C} 37-\mathrm{C} 38-\mathrm{C} 39$ & $-171.5(6)$ \\
\hline $\mathrm{N} 2 \mathrm{~B}-\mathrm{C} 37-\mathrm{C} 38-\mathrm{C} 39$ & $-178.8(8)$ \\
\hline $\mathrm{C} 37-\mathrm{C} 38-\mathrm{C} 39-\mathrm{C} 40$ & $-176.9(6)$ \\
\hline $\mathrm{C} 29 \mathrm{~B}-\mathrm{N} 2 \mathrm{~B}-\mathrm{C} 41-\mathrm{C} 42$ & $63(2)$ \\
\hline $\mathrm{C} 33 \mathrm{~B}-\mathrm{N} 2 \mathrm{~B}-\mathrm{C} 41-\mathrm{C} 42$ & $-55(3)$ \\
\hline $\mathrm{C} 37-\mathrm{N} 2 \mathrm{~B}-\mathrm{C} 41-\mathrm{C} 42$ & $-177.7(6)$ \\
\hline $\mathrm{C} 37-\mathrm{N} 2-\mathrm{C} 41-\mathrm{C} 42$ & $176.8(6)$ \\
\hline $\mathrm{C} 29-\mathrm{N} 2-\mathrm{C} 41-\mathrm{C} 42$ & $56.3(12)$ \\
\hline $\mathrm{C} 33-\mathrm{N} 2-\mathrm{C} 41-\mathrm{C} 42$ & $-64.4(12)$ \\
\hline $\mathrm{N} 2 \mathrm{~B}-\mathrm{C} 41-\mathrm{C} 42-\mathrm{C} 43$ & $170.5(8)$ \\
\hline $\mathrm{N} 2-\mathrm{C} 41-\mathrm{C} 42-\mathrm{C} 43$ & $163.0(6)$ \\
\hline $\mathrm{C} 41-\mathrm{C} 42-\mathrm{C} 43-\mathrm{C} 44$ & $55.6(9)$ \\
\hline
\end{tabular}


Hydrogen-bond geometry $\left(\AA,{ }^{\circ}\right)$

\begin{tabular}{lllll}
\hline$D-\mathrm{H} \cdots A$ & $D-\mathrm{H}$ & $\mathrm{H} \cdots A$ & $D \cdots A$ & $D-\mathrm{H} \cdots A$ \\
\hline $\mathrm{O} 6-\mathrm{H} 6 O \cdots \mathrm{O} 3^{\mathrm{i}}$ & $0.88(7)$ & $1.76(7)$ & $2.633(5)$ & $170(7)$ \\
$\mathrm{O} 8-\mathrm{H} 8 O \cdots \mathrm{O} 4$ & $0.85(8)$ & $1.75(8)$ & $2.587(5)$ & $174(9)$ \\
\hline
\end{tabular}

Symmetry code: (i) $x+1 / 2,-y+3 / 2,-z+1$.

Bis(tetra- $n$-butylammonium) $\mu$-oxalato-bis[bis(1,1,1,4,4,4-hexafluorobut-2-ene-2,3-dithiolato)molybdate(IV)] (k10171_sq)

Crystal data

$\left(\mathrm{C}_{16} \mathrm{H}_{36} \mathrm{~N}\right)\left[\mathrm{Mo}_{2}\left(\mathrm{C}_{4} \mathrm{~F}_{6} \mathrm{~S}_{2}\right)_{4}\left(\mathrm{C}_{2} \mathrm{O}_{4}\right)\right]$

$M_{r}=1669.45$

Monoclinic, $P 2{ }_{1} / n$

$a=14.2347(15) \AA$

$b=19.4940(19) \AA$

$c=14.4056(14) \AA$

$\beta=103.159(5)^{\circ}$

$V=3892.5(7) \AA^{3}$

$Z=2$

$F(000)=1692$

$D_{\mathrm{x}}=1.424 \mathrm{Mg} \mathrm{m}^{-3}$

Mo $K \alpha$ radiation, $\lambda=0.71073 \AA$

Cell parameters from 18892 reflections

$\theta=2.6-25.7^{\circ}$

$\mu=0.63 \mathrm{~mm}^{-1}$

$T=150 \mathrm{~K}$

Plate, green

$0.18 \times 0.18 \times 0.06 \mathrm{~mm}$

\section{Data collection}

Nonius KappaCCD diffractometer

Radiation source: fine-focus sealed tube

Detector resolution: 9 pixels $\mathrm{mm}^{-1}$

$\varphi$ scan and $\omega$ scans with $\kappa$ offsets

Absorption correction: multi-scan

(SORTAV; Blessing, 1995)

$T_{\text {min }}=0.720, T_{\text {max }}=0.931$

18527 measured reflections

7278 independent reflections

4243 reflections with $I>2 \sigma(I)$

$R_{\text {int }}=0.066$

$\theta_{\text {max }}=25.8^{\circ}, \theta_{\min }=2.8^{\circ}$

$h=-17 \rightarrow 16$

$k=-21 \rightarrow 23$

$l=-14 \rightarrow 17$

\section{Refinement}

Refinement on $F^{2}$

Least-squares matrix: full

$R\left[F^{2}>2 \sigma\left(F^{2}\right)\right]=0.065$

$w R\left(F^{2}\right)=0.164$

$S=1.01$

7278 reflections

560 parameters

520 restraints

Hydrogen site location: inferred from neighbouring sites

$\mathrm{H}$-atom parameters constrained

$w=1 /\left[\sigma^{2}\left(F_{\mathrm{o}}{ }^{2}\right)+(0.0589 P)^{2}+6.741 P\right]$

where $P=\left(F_{\mathrm{o}}{ }^{2}+2 F_{\mathrm{c}}{ }^{2}\right) / 3$

$(\Delta / \sigma)_{\max }=0.001$

$\Delta \rho_{\max }=0.59 \mathrm{e} \AA^{-3}$

$\Delta \rho_{\min }=-0.65$ e $\AA^{-3}$

Special details

Geometry. All esds (except the esd in the dihedral angle between two 1.s. planes) are estimated using the full covariance matrix. The cell esds are taken into account individually in the estimation of esds in distances, angles and torsion angles; correlations between esds in cell parameters are only used when they are defined by crystal symmetry. An approximate (isotropic) treatment of cell esds is used for estimating esds involving 1.s. planes.

Fractional atomic coordinates and isotropic or equivalent isotropic displacement parameters $\left(\AA^{2}\right)$

\begin{tabular}{llllll}
\hline & $x$ & $y$ & $z$ & $U_{\text {iso }} * / U_{\text {eq }}$ & Occ. $(<1)$ \\
\hline Mo1 & $0.87109(4)$ & $0.08321(2)$ & $0.36570(4)$ & $0.05150(18)$ & \\
S1 & $0.89301(13)$ & $0.07056(8)$ & $0.21225(13)$ & $0.0659(5)$ &
\end{tabular}




\begin{tabular}{|c|c|c|c|c|c|}
\hline S2 & $0.93704(12)$ & $0.19051(8)$ & $0.35112(13)$ & $0.0678(5)$ & \\
\hline S3 & $0.77245(12)$ & $0.14498(8)$ & $0.44228(12)$ & $0.0633(4)$ & \\
\hline S4 & $0.72826(11)$ & $0.02567(7)$ & $0.30266(12)$ & $0.0591(4)$ & \\
\hline $\mathrm{F} 1$ & $0.9664(5)$ & $0.0777(3)$ & $0.0486(4)$ & 0.1268 (19) & \\
\hline $\mathrm{F} 2$ & $0.9059(5)$ & $0.1775(3)$ & 0.0199 (4) & $0.139(2)$ & \\
\hline F3 & 1.0559 (4) & $0.1657(3)$ & $0.0798(5)$ & $0.141(2)$ & \\
\hline F4 & $1.1100(4)$ & $0.2576(3)$ & $0.2483(6)$ & $0.203(4)$ & \\
\hline F5 & $0.9905(5)$ & $0.3134(3)$ & $0.2739(4)$ & $0.132(2)$ & \\
\hline F6 & $0.9926(4)$ & $0.2848(2)$ & $0.1371(4)$ & $0.1192(18)$ & \\
\hline F7 & $0.6143(4)$ & $0.2064(3)$ & $0.4933(5)$ & $0.155(3)$ & \\
\hline F8 & $0.5004(4)$ & $0.1532(3)$ & $0.4101(4)$ & $0.138(2)$ & \\
\hline F9 & $0.5744(4)$ & $0.1101(3)$ & $0.5375(4)$ & $0.131(2)$ & \\
\hline F10 & $0.4944(4)$ & $0.0160(3)$ & $0.3889(5)$ & $0.146(2)$ & \\
\hline F11 & $0.5485(3)$ & $-0.0406(2)$ & $0.2910(4)$ & 0.1217 (19) & \\
\hline F12 & $0.4847(3)$ & $0.0548(3)$ & 0.2499 (4) & $0.130(2)$ & \\
\hline $\mathrm{O} 1$ & $0.9309(3)$ & $-0.01919(18)$ & $0.3874(3)$ & $0.0557(10)$ & \\
\hline $\mathrm{O} 2$ & $1.0310(3)$ & $-0.08488(18)$ & 0.4949 (3) & $0.0573(10)$ & \\
\hline $\mathrm{C} 1$ & $0.9504(5)$ & $0.1439(4)$ & $0.1820(6)$ & $0.075(2)$ & \\
\hline $\mathrm{C} 2$ & $0.9686(5)$ & $0.1961(3)$ & $0.2414(6)$ & 0.0709 (19) & \\
\hline $\mathrm{C} 3$ & $0.9702(7)$ & $0.1418(5)$ & $0.0815(7)$ & $0.102(3)$ & \\
\hline $\mathrm{C} 4$ & 1.0177 (6) & $0.2622(4)$ & $0.2255(8)$ & $0.099(3)$ & \\
\hline $\mathrm{C} 5$ & $0.6576(5)$ & $0.1110(3)$ & $0.4153(5)$ & $0.0640(17)$ & \\
\hline C6 & $0.6378(4)$ & $0.0580(3)$ & $0.3530(5)$ & $0.0636(17)$ & \\
\hline $\mathrm{C} 7$ & $0.5880(6)$ & $0.1450(4)$ & $0.4666(7)$ & $0.085(2)$ & \\
\hline $\mathrm{C} 8$ & $0.5414(5)$ & $0.0231(4)$ & $0.3193(7)$ & $0.084(2)$ & \\
\hline C9 & $0.9890(4)$ & $-0.0297(3)$ & $0.4657(4)$ & $0.0516(15)$ & \\
\hline N1 & $0.6284(10)$ & $0.2680(7)$ & $0.1575(11)$ & 0.0755 (19) & $0.589(6)$ \\
\hline $\mathrm{C} 10$ & $0.5205(11)$ & $0.2799(9)$ & $0.1500(13)$ & $0.082(3)$ & $0.589(6)$ \\
\hline H10A & 0.5095 & 0.3299 & 0.1530 & $0.099 *$ & $0.589(6)$ \\
\hline H10B & 0.4850 & 0.2639 & 0.0864 & $0.099^{*}$ & $0.589(6)$ \\
\hline $\mathrm{C} 11$ & $0.4765(9)$ & $0.2454(7)$ & $0.2247(11)$ & $0.093(3)$ & $0.589(6)$ \\
\hline H11A & 0.5043 & 0.2657 & 0.2880 & $0.111 *$ & $0.589(6)$ \\
\hline H11B & 0.4927 & 0.1959 & 0.2276 & $0.111^{*}$ & $0.589(6)$ \\
\hline $\mathrm{C} 12$ & $0.3656(10)$ & $0.2541(8)$ & $0.2012(13)$ & $0.103(4)$ & $0.589(6)$ \\
\hline $\mathrm{H} 12 \mathrm{~A}$ & 0.3423 & 0.2547 & 0.2609 & $0.124^{*}$ & $0.589(6)$ \\
\hline H12B & 0.3477 & 0.2981 & 0.1677 & $0.124 *$ & $0.589(6)$ \\
\hline C13 & $0.3202(13)$ & $0.1967(10)$ & $0.1402(14)$ & $0.133(5)$ & $0.589(6)$ \\
\hline $\mathrm{H} 13 \mathrm{~A}$ & 0.2500 & 0.2023 & 0.1254 & $0.199^{*}$ & $0.589(6)$ \\
\hline H13B & 0.3430 & 0.1966 & 0.0810 & $0.199^{*}$ & $0.589(6)$ \\
\hline $\mathrm{H} 13 \mathrm{C}$ & 0.3376 & 0.1533 & 0.1740 & $0.199 *$ & $0.589(6)$ \\
\hline C14 & $0.6590(13)$ & $0.3140(12)$ & $0.085(2)$ & $0.077(2)$ & $0.589(6)$ \\
\hline $\mathrm{H} 14 \mathrm{~A}$ & 0.6287 & 0.3595 & 0.0881 & $0.093^{*}$ & $0.589(6)$ \\
\hline H14B & 0.6325 & 0.2948 & 0.0212 & $0.093^{*}$ & $0.589(6)$ \\
\hline C15 & $0.7680(12)$ & $0.3248(10)$ & $0.0967(12)$ & $0.084(3)$ & $0.589(6)$ \\
\hline $\mathrm{H} 15 \mathrm{~A}$ & 0.7960 & 0.3428 & 0.1613 & $0.101 *$ & $0.589(6)$ \\
\hline H15B & 0.7989 & 0.2800 & 0.0903 & $0.101^{*}$ & $0.589(6)$ \\
\hline $\mathrm{C} 16$ & $0.7904(11)$ & $0.3743(7)$ & $0.0232(9)$ & 0.085 & $0.589(6)$ \\
\hline H16A & 0.8606 & 0.3831 & 0.0362 & $0.101^{*}$ & $0.589(6)$ \\
\hline
\end{tabular}




\begin{tabular}{|c|c|c|c|c|c|}
\hline H16B & 0.7569 & 0.4185 & 0.0263 & $0.101^{*}$ & $0.589(6)$ \\
\hline $\mathrm{C} 17$ & $0.7553(12)$ & $0.3416(8)$ & $-0.0780(10)$ & $0.106(4)$ & $0.589(6)$ \\
\hline H17A & 0.7692 & 0.3730 & -0.1262 & $0.159^{*}$ & $0.589(6)$ \\
\hline H17B & 0.7890 & 0.2981 & -0.0806 & $0.159^{*}$ & $0.589(6)$ \\
\hline $\mathrm{H} 17 \mathrm{C}$ & 0.6856 & 0.3333 & -0.0904 & $0.159^{*}$ & $0.589(6)$ \\
\hline C18 & $0.6467(12)$ & $0.1923(8)$ & $0.1431(11)$ & 0.085 & $0.589(6)$ \\
\hline H18A & 0.6054 & 0.1647 & 0.1757 & $0.102 *$ & $0.589(6)$ \\
\hline H18B & 0.7147 & 0.1816 & 0.1735 & $0.102 *$ & $0.589(6)$ \\
\hline C19 & $0.6260(11)$ & $0.1704(7)$ & $0.0352(11)$ & $0.094(3)$ & $0.589(6)$ \\
\hline H19A & 0.5568 & 0.1771 & 0.0053 & $0.113 *$ & $0.589(6)$ \\
\hline H19B & 0.6642 & 0.1994 & 0.0009 & $0.113 *$ & $0.589(6)$ \\
\hline $\mathrm{C} 20$ & $0.6534(11)$ & $0.0941(7)$ & $0.0276(13)$ & $0.103(4)$ & $0.589(6)$ \\
\hline $\mathrm{H} 20 \mathrm{~A}$ & 0.6589 & 0.0714 & 0.0901 & $0.123^{*}$ & $0.589(6)$ \\
\hline $\mathrm{H} 20 \mathrm{~B}$ & 0.7168 & 0.0910 & 0.0106 & $0.123^{*}$ & $0.589(6)$ \\
\hline C21 & $0.5731(13)$ & $0.0556(9)$ & $-0.0519(14)$ & $0.123(6)$ & $0.589(6)$ \\
\hline $\mathrm{H} 21 \mathrm{~A}$ & 0.5914 & 0.0074 & -0.0558 & $0.185^{*}$ & $0.589(6)$ \\
\hline $\mathrm{H} 21 \mathrm{~B}$ & 0.5105 & 0.0582 & -0.0344 & $0.185^{*}$ & $0.589(6)$ \\
\hline $\mathrm{H} 21 \mathrm{C}$ & 0.5684 & 0.0778 & -0.1138 & $0.185^{*}$ & $0.589(6)$ \\
\hline C22 & $0.6825(19)$ & $0.2873(10)$ & $0.2593(14)$ & $0.082(3)$ & $0.589(6)$ \\
\hline $\mathrm{H} 22 \mathrm{~A}$ & 0.6636 & 0.2545 & 0.3043 & $0.099^{*}$ & $0.589(6)$ \\
\hline $\mathrm{H} 22 \mathrm{~B}$ & 0.7525 & 0.2814 & 0.2640 & $0.099^{*}$ & $0.589(6)$ \\
\hline $\mathrm{C} 23$ & $0.666(3)$ & $0.3588(11)$ & $0.2913(16)$ & $0.090(3)$ & $0.589(6)$ \\
\hline $\mathrm{H} 23 \mathrm{~A}$ & 0.5955 & 0.3663 & 0.2812 & $0.108^{*}$ & $0.589(6)$ \\
\hline $\mathrm{H} 23 \mathrm{~B}$ & 0.6905 & 0.3917 & 0.2501 & $0.108^{*}$ & $0.589(6)$ \\
\hline $\mathrm{C} 24$ & $0.7128(13)$ & $0.3750(9)$ & $0.3947(13)$ & $0.103(3)$ & $0.589(6)$ \\
\hline $\mathrm{H} 24 \mathrm{~A}$ & 0.6894 & 0.4200 & 0.4118 & $0.123 *$ & $0.589(6)$ \\
\hline $\mathrm{H} 24 \mathrm{~B}$ & 0.6936 & 0.3398 & 0.4364 & $0.123 *$ & $0.589(6)$ \\
\hline $\mathrm{C} 25$ & $0.8214(11)$ & $0.3767(8)$ & $0.4118(12)$ & $0.120(5)$ & $0.589(6)$ \\
\hline $\mathrm{H} 25 \mathrm{~A}$ & 0.8492 & 0.3872 & 0.4789 & $0.180^{*}$ & $0.589(6)$ \\
\hline $\mathrm{H} 25 \mathrm{~B}$ & 0.8449 & 0.3319 & 0.3960 & $0.180^{*}$ & $0.589(6)$ \\
\hline $\mathrm{H} 25 \mathrm{C}$ & 0.8407 & 0.4121 & 0.3715 & $0.180^{*}$ & $0.589(6)$ \\
\hline N1A & $0.6310(15)$ & $0.2661(10)$ & $0.1504(15)$ & $0.077(2)$ & $0.411(6)$ \\
\hline $\mathrm{C} 10 \mathrm{~A}$ & $0.5216(14)$ & $0.2704(13)$ & $0.1279(19)$ & $0.082(3)$ & $0.411(6)$ \\
\hline $\mathrm{H} 10 \mathrm{C}$ & 0.5033 & 0.3171 & 0.1443 & $0.098^{*}$ & $0.411(6)$ \\
\hline H10D & 0.4974 & 0.2645 & 0.0583 & $0.098^{*}$ & $0.411(6)$ \\
\hline $\mathrm{C} 11 \mathrm{~A}$ & $0.4713(12)$ & $0.2192(10)$ & $0.1781(16)$ & 0.090 & $0.411(6)$ \\
\hline $\mathrm{H} 11 \mathrm{C}$ & 0.4948 & 0.2238 & 0.2480 & $0.108^{*}$ & $0.411(6)$ \\
\hline H11D & 0.4853 & 0.1720 & 0.1598 & $0.108^{*}$ & $0.411(6)$ \\
\hline $\mathrm{C} 12 \mathrm{~A}$ & 0.3619 (13) & $0.2325(13)$ & $0.1502(17)$ & 0.095 (4) & $0.411(6)$ \\
\hline $\mathrm{H} 12 \mathrm{C}$ & 0.3491 & 0.2825 & 0.1482 & $0.114^{*}$ & $0.411(6)$ \\
\hline H12D & 0.3344 & 0.2132 & 0.0862 & $0.114^{*}$ & $0.411(6)$ \\
\hline $\mathrm{C} 13 \mathrm{~A}$ & $0.3161(15)$ & $0.1988(14)$ & $0.2233(19)$ & $0.122(6)$ & $0.411(6)$ \\
\hline H13D & 0.2463 & 0.2068 & 0.2064 & $0.183^{*}$ & $0.411(6)$ \\
\hline H13E & 0.3288 & 0.1493 & 0.2245 & $0.183^{*}$ & $0.411(6)$ \\
\hline $\mathrm{H} 13 \mathrm{~F}$ & 0.3434 & 0.2183 & 0.2863 & $0.183^{*}$ & $0.411(6)$ \\
\hline $\mathrm{C} 14 \mathrm{~A}$ & $0.6599(19)$ & $0.3197(17)$ & $0.086(3)$ & 0.078 & $0.411(6)$ \\
\hline $\mathrm{H} 14 \mathrm{C}$ & 0.6508 & 0.3659 & 0.1114 & $0.094 *$ & $0.411(6)$ \\
\hline H14D & 0.6174 & 0.3159 & 0.0218 & $0.094 *$ & $0.411(6)$ \\
\hline
\end{tabular}




\begin{tabular}{|c|c|c|c|c|c|}
\hline $\mathrm{C} 15 \mathrm{~A}$ & $0.7649(17)$ & $0.3117(14)$ & $0.0793(17)$ & $0.086(3)$ & $0.411(6)$ \\
\hline $\mathrm{H} 15 \mathrm{C}$ & 0.8074 & 0.3313 & 0.1373 & $0.103^{*}$ & $0.411(6)$ \\
\hline H15D & 0.7800 & 0.2622 & 0.0775 & $0.103 *$ & $0.411(6)$ \\
\hline $\mathrm{C} 16 \mathrm{~A}$ & $0.7871(17)$ & $0.3454(12)$ & $-0.0055(18)$ & $0.096(4)$ & $0.411(6)$ \\
\hline $\mathrm{H} 16 \mathrm{C}$ & 0.7799 & 0.3105 & -0.0566 & $0.116^{*}$ & $0.411(6)$ \\
\hline H16D & 0.8559 & 0.3589 & 0.0112 & $0.116^{*}$ & $0.411(6)$ \\
\hline C17A & $0.7295(17)$ & $0.4075(11)$ & $-0.0469(17)$ & $0.122(6)$ & $0.411(6)$ \\
\hline H17D & 0.7522 & 0.4239 & -0.1022 & $0.182 *$ & $0.411(6)$ \\
\hline H17E & 0.6611 & 0.3951 & -0.0668 & $0.182 *$ & $0.411(6)$ \\
\hline $\mathrm{H} 17 \mathrm{~F}$ & 0.7376 & 0.4437 & 0.0014 & $0.182 *$ & $0.411(6)$ \\
\hline $\mathrm{C} 18 \mathrm{~A}$ & $0.6668(17)$ & $0.1949(11)$ & $0.1299(17)$ & 0.085 & $0.411(6)$ \\
\hline $\mathrm{H} 18 \mathrm{C}$ & 0.6748 & 0.1657 & 0.1875 & $0.101 *$ & $0.411(6)$ \\
\hline H18D & 0.7303 & 0.1989 & 0.1131 & $0.101 *$ & $0.411(6)$ \\
\hline C19A & $0.5922(15)$ & $0.1613(10)$ & $0.0455(16)$ & 0.093 (4) & $0.411(6)$ \\
\hline $\mathrm{H} 19 \mathrm{C}$ & 0.5261 & 0.1680 & 0.0551 & $0.112^{*}$ & $0.411(6)$ \\
\hline H19D & 0.5962 & 0.1837 & -0.0151 & $0.112 *$ & $0.411(6)$ \\
\hline $\mathrm{C} 20 \mathrm{~A}$ & $0.6129(17)$ & $0.0838(10)$ & $0.0397(16)$ & $0.100(4)$ & $0.411(6)$ \\
\hline $\mathrm{H} 20 \mathrm{C}$ & 0.5592 & 0.0566 & 0.0541 & $0.119^{*}$ & $0.411(6)$ \\
\hline $\mathrm{H} 20 \mathrm{D}$ & 0.6730 & 0.0715 & 0.0863 & $0.119 *$ & $0.411(6)$ \\
\hline $\mathrm{C} 21 \mathrm{~A}$ & $0.6239(17)$ & $0.0679(13)$ & $-0.0686(16)$ & $0.119(7)$ & $0.411(6)$ \\
\hline $\mathrm{H} 21 \mathrm{D}$ & 0.6371 & 0.0190 & -0.0748 & $0.179 *$ & $0.411(6)$ \\
\hline $\mathrm{H} 21 \mathrm{E}$ & 0.5640 & 0.0802 & -0.1141 & $0.179 *$ & $0.411(6)$ \\
\hline $\mathrm{H} 21 \mathrm{~F}$ & 0.6773 & 0.0950 & -0.0820 & $0.179 *$ & $0.411(6)$ \\
\hline $\mathrm{C} 22 \mathrm{~A}$ & $0.676(3)$ & $0.2786(15)$ & $0.2551(19)$ & $0.082(3)$ & $0.411(6)$ \\
\hline $\mathrm{H} 22 \mathrm{C}$ & 0.6483 & 0.2451 & 0.2928 & $0.099 *$ & $0.411(6)$ \\
\hline $\mathrm{H} 22 \mathrm{D}$ & 0.7461 & 0.2682 & 0.2658 & $0.099 *$ & $0.411(6)$ \\
\hline $\mathrm{C} 23 \mathrm{~A}$ & $0.666(4)$ & $0.3485(15)$ & $0.295(2)$ & $0.090(3)$ & $0.411(6)$ \\
\hline $\mathrm{H} 23 \mathrm{C}$ & 0.5981 & 0.3576 & 0.2950 & $0.108^{*}$ & $0.411(6)$ \\
\hline $\mathrm{H} 23 \mathrm{D}$ & 0.6884 & 0.3838 & 0.2552 & $0.108 *$ & $0.411(6)$ \\
\hline C24A & $0.728(2)$ & $0.3515(10)$ & $0.3967(19)$ & $0.100(4)$ & $0.411(6)$ \\
\hline $\mathrm{H} 24 \mathrm{C}$ & 0.7068 & 0.3153 & 0.4356 & $0.120^{*}$ & $0.411(6)$ \\
\hline $\mathrm{H} 24 \mathrm{D}$ & 0.7963 & 0.3428 & 0.3961 & $0.120 *$ & $0.411(6)$ \\
\hline $\mathrm{C} 25 \mathrm{~A}$ & $0.7188(16)$ & 0.4207 (9) & $0.4402(15)$ & $0.102(5)$ & $0.411(6)$ \\
\hline $\mathrm{H} 25 \mathrm{D}$ & 0.7586 & 0.4219 & 0.5054 & $0.153^{*}$ & $0.411(6)$ \\
\hline $\mathrm{H} 25 \mathrm{E}$ & 0.7406 & 0.4564 & 0.4020 & $0.153^{*}$ & $0.411(6)$ \\
\hline $\mathrm{H} 25 \mathrm{~F}$ & 0.6512 & 0.4289 & 0.4416 & $0.153^{*}$ & $0.411(6)$ \\
\hline
\end{tabular}

Atomic displacement parameters $\left(\AA^{2}\right)$

\begin{tabular}{lllllll}
\hline & $U^{11}$ & $U^{22}$ & $U^{33}$ & $U^{12}$ & $U^{13}$ & $U^{23}$ \\
\hline Mo1 & $0.0487(3)$ & $0.0358(3)$ & $0.0643(3)$ & $0.0027(2)$ & $0.0008(2)$ & $0.0017(2)$ \\
S1 & $0.0684(10)$ & $0.0535(9)$ & $0.0773(11)$ & $0.0068(8)$ & $0.0199(9)$ & $0.0045(8)$ \\
S2 & $0.0624(10)$ & $0.0432(8)$ & $0.0890(13)$ & $-0.0048(8)$ & $-0.0006(9)$ & $0.0076(8)$ \\
S3 & $0.0642(10)$ & $0.0487(9)$ & $0.0731(11)$ & $0.0066(8)$ & $0.0075(9)$ & $-0.0029(8)$ \\
S4 & $0.0544(9)$ & $0.0455(8)$ & $0.0722(11)$ & $-0.0018(7)$ & $0.0037(8)$ & $-0.0027(8)$ \\
F1 & $0.178(5)$ & $0.104(4)$ & $0.122(4)$ & $0.017(4)$ & $0.085(4)$ & $0.010(3)$ \\
F2 & $0.174(5)$ & $0.151(5)$ & $0.109(4)$ & $0.072(4)$ & $0.067(4)$ & $0.053(4)$ \\
F3 & $0.121(4)$ & $0.136(5)$ & $0.198(6)$ & $0.014(4)$ & $0.102(4)$ & $0.039(4)$
\end{tabular}




\begin{tabular}{|c|c|c|c|c|c|c|}
\hline F4 & $0.060(3)$ & $0.134(5)$ & $0.384(11)$ & $-0.021(3)$ & $-0.014(4)$ & $0.136(6)$ \\
\hline F5 & $0.170(5)$ & $0.069(3)$ & $0.153(5)$ & $-0.045(3)$ & $0.031(4)$ & $0.017(3)$ \\
\hline F6 & $0.106(4)$ & $0.095(3)$ & $0.167(5)$ & $0.004(3)$ & $0.053(3)$ & $0.058(4)$ \\
\hline F7 & $0.159(5)$ & $0.079(3)$ & $0.270(8)$ & $-0.004(3)$ & $0.141(5)$ & $-0.048(4)$ \\
\hline F8 & $0.106(4)$ & $0.177(6)$ & $0.145(5)$ & $0.062(4)$ & $0.060(4)$ & $0.034(4)$ \\
\hline F9 & $0.194(6)$ & $0.100(3)$ & $0.134(4)$ & $0.045(4)$ & $0.106(4)$ & $0.038(3)$ \\
\hline F10 & $0.108(4)$ & $0.167(6)$ & $0.174(6)$ & $-0.052(4)$ & $0.057(4)$ & $0.002(4)$ \\
\hline F11 & $0.073(3)$ & $0.067(3)$ & $0.208(6)$ & $-0.018(2)$ & $-0.005(3)$ & -0.008 \\
\hline F12 & $0.077(3)$ & $0.096(3)$ & $0.187(5)$ & $-0.022(3)$ & -0.033 & $0.049(4)$ \\
\hline $\mathrm{O} 1$ & $0.054(2)$ & $0.038(2)$ & $0.066(3)$ & 0.0067 (18) & $-0.005(2)$ & $-0.0040(19)$ \\
\hline $\mathrm{O} 2$ & $0.056(2)$ & $0.032(2)$ & $0.073(3)$ & 0.0048 (19) & $-0.007(2)$ & $-0.0031(19)$ \\
\hline $\mathrm{C} 1$ & $0.061(4)$ & $0.070(5)$ & $0.098(6)$ & $0.011(4)$ & $0.029(4)$ & $0.027(4)$ \\
\hline $\mathrm{C} 2$ & $0.055(4)$ & $0.055(4)$ & $0.100(6)$ & $-0.001(3)$ & $0.012(4)$ & $0.019(4)$ \\
\hline $\mathrm{C} 3$ & $0.107(7)$ & $0.088(6)$ & $0.129(8)$ & $0.022(6)$ & $0.064(6)$ & $0.028(6)$ \\
\hline $\mathrm{C} 4$ & $0.074(6)$ & $0.068(5)$ & $0.146(9)$ & $0.004(4)$ & $0.009(5)$ & $0.038(6)$ \\
\hline $\mathrm{C} 5$ & $0.064(4)$ & $0.054(4)$ & $0.077(5)$ & 0.009 (3) & $0.022(4)$ & $0.015(3)$ \\
\hline C6 & $0.055(4)$ & $0.050(3)$ & $0.083(5)$ & $0.001(3)$ & $0.010(3)$ & $0.014(3)$ \\
\hline $\mathrm{C} 7$ & $0.094(6)$ & $0.063(5)$ & $0.110(7)$ & $0.017(4)$ & $0.049(5)$ & $0.018(5)$ \\
\hline $\mathrm{C} 8$ & $0.056(4)$ & $0.069(5)$ & $0.126(7)$ & $-0.003(4)$ & $0.018(5)$ & $0.013(5)$ \\
\hline C9 & $0.042(3)$ & $0.038(3)$ & $0.072(4)$ & $-0.001(3)$ & $0.006(3)$ & $0.000(3)$ \\
\hline N1 & $0.056(3)$ & $0.057(3)$ & $0.104(4)$ & $0.003(3)$ & $-0.003(3)$ & $0.023(3)$ \\
\hline $\mathrm{C} 10$ & $0.060(4)$ & $0.066(5)$ & $0.112(6)$ & $-0.002(4)$ & $0.000(4)$ & $0.023(5)$ \\
\hline C11 & $0.069(4)$ & $0.076(6)$ & $0.125(7)$ & $-0.010(5)$ & $0.005(5)$ & $0.021(5)$ \\
\hline C12 & $0.075(5)$ & $0.095(7)$ & $0.132(8)$ & $-0.014(5)$ & $0.006(6)$ & $0.020(6)$ \\
\hline C13 & $0.098(9)$ & $0.139(10)$ & 0.145 (11) & $0.017(8)$ & $-0.008(10)$ & $-0.022(10)$ \\
\hline $\mathrm{C} 14$ & $0.062(4)$ & $0.066(5)$ & $0.100(5)$ & 0.007 (4) & $0.012(4)$ & $0.015(4)$ \\
\hline $\mathrm{C} 15$ & $0.073(4)$ & $0.076(6)$ & $0.102(5)$ & $0.011(4)$ & $0.019(5)$ & $0.001(5)$ \\
\hline $\mathrm{C} 16$ & $0.077(5)$ & $0.079(6)$ & $0.098(6)$ & $0.008(5)$ & $0.023(5)$ & $-0.012(5)$ \\
\hline $\mathrm{C} 17$ & $0.112(9)$ & $0.117(9)$ & $0.091(9)$ & $-0.016(8)$ & $0.025(8)$ & $-0.019(8)$ \\
\hline $\mathrm{C} 18$ & $0.066(5)$ & $0.060(4)$ & $0.114(5)$ & $0.006(4)$ & $-0.011(4)$ & $0.013(4)$ \\
\hline C19 & $0.068(6)$ & $0.068(5)$ & $0.125(5)$ & $0.001(5)$ & $-0.023(5)$ & $0.005(5)$ \\
\hline $\mathrm{C} 20$ & $0.079(7)$ & $0.074(6)$ & $0.131(6)$ & $-0.001(6)$ & $-0.025(6)$ & $-0.004(5)$ \\
\hline $\mathrm{C} 21$ & $0.104(11)$ & $0.094(9)$ & $0.147(10)$ & $-0.005(9)$ & $-0.021(9)$ & $-0.012(8)$ \\
\hline $\mathrm{C} 22$ & $0.071(4)$ & $0.064(5)$ & $0.102(5)$ & $0.003(4)$ & $-0.003(4)$ & $0.024(4)$ \\
\hline $\mathrm{C} 23$ & $0.086(5)$ & $0.072(6)$ & $0.101(5)$ & $0.008(5)$ & $0.001(4)$ & $0.021(5)$ \\
\hline $\mathrm{C} 24$ & $0.103(6)$ & $0.080(7)$ & $0.111(6)$ & $0.012(6)$ & $-0.003(5)$ & $0.021(6)$ \\
\hline $\mathrm{C} 25$ & $0.128(9)$ & $0.098(9)$ & $0.121(9)$ & $0.001(8)$ & $-0.001(9)$ & $-0.009(8)$ \\
\hline N1A & $0.057(3)$ & $0.059(4)$ & $0.104(4)$ & $0.003(3)$ & $-0.002(3)$ & $0.021(3)$ \\
\hline $\mathrm{C} 10 \mathrm{~A}$ & $0.059(4)$ & $0.065(5)$ & $0.113(6)$ & $0.000(4)$ & $0.001(5)$ & $0.024(5)$ \\
\hline C11A & $0.066(5)$ & $0.076(6)$ & $0.118(7)$ & -0.008 & $0.003(6)$ & $0.023(5)$ \\
\hline $\mathrm{C} 12 \mathrm{~A}$ & $0.069(6)$ & $0.082(7)$ & $0.126(8)$ & $-0.010(6)$ & $0.005(7)$ & $0.015(7)$ \\
\hline C13A & $0.087(10)$ & $0.129(11)$ & $0.144(12)$ & $0.005(9)$ & $0.015(11)$ & $0.026(11)$ \\
\hline $\mathrm{C} 14 \mathrm{~A}$ & $0.063(4)$ & $0.066(5)$ & $0.101(5)$ & $0.006(4)$ & $0.010(4)$ & $0.016(5)$ \\
\hline $\mathrm{C} 15 \mathrm{~A}$ & $0.073(5)$ & $0.078(6)$ & $0.105(6)$ & $0.010(5)$ & $0.019(5)$ & $0.005(5)$ \\
\hline $\mathrm{C} 16 \mathrm{~A}$ & $0.085(6)$ & $0.094(7)$ & $0.110(7)$ & $0.012(6)$ & $0.021(6)$ & $0.005(6)$ \\
\hline C17A & $0.122(11)$ & $0.126(11)$ & 0.119 (11) & $0.002(10)$ & $0.032(10)$ & $0.012(10)$ \\
\hline C18A & $0.065(5)$ & $0.058(5)$ & $0.114(5)$ & $0.004(5)$ & $-0.012(5)$ & $0.014(5)$ \\
\hline C19A & $0.072(7)$ & $0.066(5)$ & $0.122(6)$ & $0.000(5)$ & $-0.019(6)$ & $0.008(5)$ \\
\hline
\end{tabular}




\begin{tabular}{lllllll} 
& & & & & \\
$\mathrm{C} 20 \mathrm{~A}$ & $0.074(8)$ & $0.073(6)$ & $0.128(7)$ & $-0.001(6)$ & $-0.025(7)$ & $0.000(6)$ \\
$\mathrm{C} 21 \mathrm{~A}$ & $0.080(12)$ & $0.099(10)$ & $0.153(11)$ & $0.006(10)$ & $-0.026(11)$ & $-0.015(10)$ \\
$\mathrm{C} 22 \mathrm{~A}$ & $0.071(5)$ & $0.064(5)$ & $0.102(5)$ & $0.005(5)$ & $-0.002(4)$ & $0.022(5)$ \\
$\mathrm{C} 23 \mathrm{~A}$ & $0.086(5)$ & $0.071(6)$ & $0.103(5)$ & $0.007(6)$ & $0.000(5)$ & $0.022(5)$ \\
$\mathrm{C} 24 \mathrm{~A}$ & $0.101(6)$ & $0.077(7)$ & $0.111(6)$ & $0.009(6)$ & $-0.002(6)$ & $0.020(7)$ \\
$\mathrm{C} 25 \mathrm{~A}$ & $0.127(11)$ & $0.069(9)$ & $0.101(10)$ & $-0.001(9)$ & $0.008(9)$ & $0.002(9)$ \\
\hline
\end{tabular}

Geometric parameters $\left(\AA,{ }^{o}\right)$

\begin{tabular}{|c|c|c|c|}
\hline Mo1-O1 & $2.165(4)$ & $\mathrm{C} 20-\mathrm{H} 20 \mathrm{~B}$ & 0.9900 \\
\hline $\mathrm{Mo} 1-\mathrm{O} 2^{\mathrm{i}}$ & $2.168(4)$ & $\mathrm{C} 21-\mathrm{H} 21 \mathrm{~A}$ & 0.9800 \\
\hline Mo1-S3 & $2.3116(18)$ & $\mathrm{C} 21-\mathrm{H} 21 \mathrm{~B}$ & 0.9800 \\
\hline Mo1-S1 & $2.3148(19)$ & $\mathrm{C} 21-\mathrm{H} 21 \mathrm{C}$ & 0.9800 \\
\hline Mo1-S4 & $2.3186(16)$ & $\mathrm{C} 22-\mathrm{C} 23$ & $1.503(13)$ \\
\hline Mo1-S2 & $2.3216(16)$ & $\mathrm{C} 22-\mathrm{H} 22 \mathrm{~A}$ & 0.9900 \\
\hline $\mathrm{S} 1-\mathrm{C} 1$ & $1.749(7)$ & $\mathrm{C} 22-\mathrm{H} 22 \mathrm{~B}$ & 0.9900 \\
\hline $\mathrm{S} 2-\mathrm{C} 2$ & $1.742(8)$ & $\mathrm{C} 23-\mathrm{C} 24$ & $1.520(14)$ \\
\hline $\mathrm{S} 3-\mathrm{C} 5$ & $1.724(7)$ & $\mathrm{C} 23-\mathrm{H} 23 \mathrm{~A}$ & 0.9900 \\
\hline $\mathrm{S} 4-\mathrm{C} 6$ & $1.734(7)$ & $\mathrm{C} 23-\mathrm{H} 23 \mathrm{~B}$ & 0.9900 \\
\hline $\mathrm{F} 1-\mathrm{C} 3$ & $1.333(10)$ & $\mathrm{C} 24-\mathrm{C} 25$ & $1.51(2)$ \\
\hline $\mathrm{F} 2-\mathrm{C} 3$ & $1.319(10)$ & $\mathrm{C} 24-\mathrm{H} 24 \mathrm{~A}$ & 0.9900 \\
\hline $\mathrm{F} 3-\mathrm{C} 3$ & $1.312(10)$ & $\mathrm{C} 24-\mathrm{H} 24 \mathrm{~B}$ & 0.9900 \\
\hline $\mathrm{F} 4-\mathrm{C} 4$ & $1.283(9)$ & $\mathrm{C} 25-\mathrm{H} 25 \mathrm{~A}$ & 0.9800 \\
\hline $\mathrm{F} 5-\mathrm{C} 4$ & $1.325(10)$ & $\mathrm{C} 25-\mathrm{H} 25 \mathrm{~B}$ & 0.9800 \\
\hline $\mathrm{F} 6-\mathrm{C} 4$ & $1.318(10)$ & $\mathrm{C} 25-\mathrm{H} 25 \mathrm{C}$ & 0.9800 \\
\hline F7-C7 & $1.288(9)$ & $\mathrm{N} 1 \mathrm{~A}-\mathrm{C} 14 \mathrm{~A}$ & $1.512(14)$ \\
\hline $\mathrm{F} 8-\mathrm{C} 7$ & $1.335(9)$ & $\mathrm{N} 1 \mathrm{~A}-\mathrm{C} 10 \mathrm{~A}$ & $1.519(14)$ \\
\hline $\mathrm{F} 9-\mathrm{C} 7$ & $1.277(8)$ & $\mathrm{N} 1 \mathrm{~A}-\mathrm{C} 22 \mathrm{~A}$ & $1.519(14)$ \\
\hline $\mathrm{F} 10-\mathrm{C} 8$ & $1.333(9)$ & $\mathrm{N} 1 \mathrm{~A}-\mathrm{C} 18 \mathrm{~A}$ & $1.530(14)$ \\
\hline $\mathrm{F} 11-\mathrm{C} 8$ & $1.320(9)$ & $\mathrm{C} 10 \mathrm{~A}-\mathrm{C} 11 \mathrm{~A}$ & $1.507(16)$ \\
\hline $\mathrm{F} 12-\mathrm{C} 8$ & $1.290(9)$ & $\mathrm{C} 10 \mathrm{~A}-\mathrm{H} 10 \mathrm{C}$ & 0.9900 \\
\hline $\mathrm{O} 1-\mathrm{C} 9$ & $1.254(6)$ & $\mathrm{C} 10 \mathrm{~A}-\mathrm{H} 10 \mathrm{D}$ & 0.9900 \\
\hline $\mathrm{O} 2-\mathrm{C} 9$ & $1.255(6)$ & $\mathrm{C} 11 \mathrm{~A}-\mathrm{C} 12 \mathrm{~A}$ & $1.538(16)$ \\
\hline $\mathrm{O} 2-\mathrm{Mo}^{\mathrm{i}}$ & $2.168(4)$ & $\mathrm{C} 11 \mathrm{~A}-\mathrm{H} 11 \mathrm{C}$ & 0.9900 \\
\hline $\mathrm{C} 1-\mathrm{C} 2$ & $1.318(10)$ & $\mathrm{C} 11 \mathrm{~A}-\mathrm{H} 11 \mathrm{D}$ & 0.9900 \\
\hline $\mathrm{C} 1-\mathrm{C} 3$ & $1.537(11)$ & $\mathrm{C} 12 \mathrm{~A}-\mathrm{C} 13 \mathrm{~A}$ & $1.510(19)$ \\
\hline $\mathrm{C} 2-\mathrm{C} 4$ & $1.507(10)$ & $\mathrm{C} 12 \mathrm{~A}-\mathrm{H} 12 \mathrm{C}$ & 0.9900 \\
\hline $\mathrm{C} 5-\mathrm{C} 6$ & $1.357(9)$ & $\mathrm{C} 12 \mathrm{~A}-\mathrm{H} 12 \mathrm{D}$ & 0.9900 \\
\hline $\mathrm{C} 5-\mathrm{C} 7$ & $1.517(10)$ & $\mathrm{C} 13 \mathrm{~A}-\mathrm{H} 13 \mathrm{D}$ & 0.9800 \\
\hline $\mathrm{C} 6-\mathrm{C} 8$ & $1.508(9)$ & $\mathrm{C} 13 \mathrm{~A}-\mathrm{H} 13 \mathrm{E}$ & 0.9800 \\
\hline $\mathrm{C} 9-\mathrm{C} 9^{\mathrm{i}}$ & $1.509(11)$ & $\mathrm{C} 13 \mathrm{~A}-\mathrm{H} 13 \mathrm{~F}$ & 0.9800 \\
\hline $\mathrm{N} 1-\mathrm{C} 14$ & $1.509(11)$ & $\mathrm{C} 14 \mathrm{~A}-\mathrm{C} 15 \mathrm{~A}$ & $1.529(16)$ \\
\hline $\mathrm{N} 1-\mathrm{C} 18$ & $1.522(12)$ & $\mathrm{C} 14 \mathrm{~A}-\mathrm{H} 14 \mathrm{C}$ & 0.9900 \\
\hline $\mathrm{N} 1-\mathrm{C} 10$ & $1.532(11)$ & $\mathrm{C} 14 \mathrm{~A}-\mathrm{H} 14 \mathrm{D}$ & 0.9900 \\
\hline $\mathrm{N} 1-\mathrm{C} 22$ & $1.539(12)$ & $\mathrm{C} 15 \mathrm{~A}-\mathrm{C} 16 \mathrm{~A}$ & $1.483(17)$ \\
\hline $\mathrm{C} 10-\mathrm{C} 11$ & $1.519(13)$ & $\mathrm{C} 15 \mathrm{~A}-\mathrm{H} 15 \mathrm{C}$ & 0.9900 \\
\hline $\mathrm{C} 10-\mathrm{H} 10 \mathrm{~A}$ & 0.9900 & $\mathrm{C} 15 \mathrm{~A}-\mathrm{H} 15 \mathrm{D}$ & 0.9900 \\
\hline C10-H10B & 0.9900 & $\mathrm{C} 16 \mathrm{~A}-\mathrm{C} 17 \mathrm{~A}$ & $1.506(18)$ \\
\hline
\end{tabular}




\begin{tabular}{|c|c|c|c|}
\hline $\mathrm{C} 11-\mathrm{C} 12$ & $1.547(14)$ & $\mathrm{C} 16 \mathrm{~A}-\mathrm{H} 16 \mathrm{C}$ & 0.9900 \\
\hline $\mathrm{C} 11-\mathrm{H} 11 \mathrm{~A}$ & 0.9900 & $\mathrm{C} 16 \mathrm{~A}-\mathrm{H} 16 \mathrm{D}$ & 0.9900 \\
\hline $\mathrm{C} 11-\mathrm{H} 11 \mathrm{~B}$ & 0.9900 & $\mathrm{C} 17 \mathrm{~A}-\mathrm{H} 17 \mathrm{D}$ & 0.9800 \\
\hline $\mathrm{C} 12-\mathrm{C} 13$ & $1.476(16)$ & $\mathrm{C} 17 \mathrm{~A}-\mathrm{H} 17 \mathrm{E}$ & 0.9800 \\
\hline $\mathrm{C} 12-\mathrm{H} 12 \mathrm{~A}$ & 0.9900 & $\mathrm{C} 17 \mathrm{~A}-\mathrm{H} 17 \mathrm{~F}$ & 0.9800 \\
\hline $\mathrm{C} 12-\mathrm{H} 12 \mathrm{~B}$ & 0.9900 & $\mathrm{C} 18 \mathrm{~A}-\mathrm{C} 19 \mathrm{~A}$ & $1.564(17)$ \\
\hline $\mathrm{C} 13-\mathrm{H} 13 \mathrm{~A}$ & 0.9800 & $\mathrm{C} 18 \mathrm{~A}-\mathrm{H} 18 \mathrm{C}$ & 0.9900 \\
\hline C13-H13B & 0.9800 & $\mathrm{C} 18 \mathrm{~A}-\mathrm{H} 18 \mathrm{D}$ & 0.9900 \\
\hline $\mathrm{C} 13-\mathrm{H} 13 \mathrm{C}$ & 0.9800 & $\mathrm{C} 19 \mathrm{~A}-\mathrm{C} 20 \mathrm{~A}$ & $1.544(16)$ \\
\hline $\mathrm{C} 14-\mathrm{C} 15$ & $1.538(14)$ & C19A-H19C & 0.9900 \\
\hline $\mathrm{C} 14-\mathrm{H} 14 \mathrm{~A}$ & 0.9900 & C19A-H19D & 0.9900 \\
\hline C14-H14B & 0.9900 & $\mathrm{C} 20 \mathrm{~A}-\mathrm{C} 21 \mathrm{~A}$ & $1.633(19)$ \\
\hline $\mathrm{C} 15-\mathrm{C} 16$ & $1.518(14)$ & $\mathrm{C} 20 \mathrm{~A}-\mathrm{H} 20 \mathrm{C}$ & 0.9900 \\
\hline C15-H15A & 0.9900 & $\mathrm{C} 20 \mathrm{~A}-\mathrm{H} 20 \mathrm{D}$ & 0.9900 \\
\hline $\mathrm{C} 15-\mathrm{H} 15 \mathrm{~B}$ & 0.9900 & $\mathrm{C} 21 \mathrm{~A}-\mathrm{H} 21 \mathrm{D}$ & 0.9800 \\
\hline $\mathrm{C} 16-\mathrm{C} 17$ & $1.564(15)$ & $\mathrm{C} 21 \mathrm{~A}-\mathrm{H} 21 \mathrm{E}$ & 0.9800 \\
\hline $\mathrm{C} 16-\mathrm{H} 16 \mathrm{~A}$ & 0.9900 & $\mathrm{C} 21 \mathrm{~A}-\mathrm{H} 21 \mathrm{~F}$ & 0.9800 \\
\hline $\mathrm{C} 16-\mathrm{H} 16 \mathrm{~B}$ & 0.9900 & $\mathrm{C} 22 \mathrm{~A}-\mathrm{C} 23 \mathrm{~A}$ & $1.498(16)$ \\
\hline C17-H17A & 0.9800 & $\mathrm{C} 22 \mathrm{~A}-\mathrm{H} 22 \mathrm{C}$ & 0.9900 \\
\hline C17-H17B & 0.9800 & $\mathrm{C} 22 \mathrm{~A}-\mathrm{H} 22 \mathrm{D}$ & 0.9900 \\
\hline $\mathrm{C} 17-\mathrm{H} 17 \mathrm{C}$ & 0.9800 & $\mathrm{C} 23 \mathrm{~A}-\mathrm{C} 24 \mathrm{~A}$ & $1.527(17)$ \\
\hline $\mathrm{C} 18-\mathrm{C} 19$ & $1.574(15)$ & $\mathrm{C} 23 \mathrm{~A}-\mathrm{H} 23 \mathrm{C}$ & 0.9900 \\
\hline $\mathrm{C} 18-\mathrm{H} 18 \mathrm{~A}$ & 0.9900 & $\mathrm{C} 23 \mathrm{~A}-\mathrm{H} 23 \mathrm{D}$ & 0.9900 \\
\hline $\mathrm{C} 18-\mathrm{H} 18 \mathrm{~B}$ & 0.9900 & $\mathrm{C} 24 \mathrm{~A}-\mathrm{C} 25 \mathrm{~A}$ & $1.51(2)$ \\
\hline $\mathrm{C} 19-\mathrm{C} 20$ & $1.548(13)$ & $\mathrm{C} 24 \mathrm{~A}-\mathrm{H} 24 \mathrm{C}$ & 0.9900 \\
\hline $\mathrm{C} 19-\mathrm{H} 19 \mathrm{~A}$ & 0.9900 & $\mathrm{C} 24 \mathrm{~A}-\mathrm{H} 24 \mathrm{D}$ & 0.9900 \\
\hline C19-H19B & 0.9900 & $\mathrm{C} 25 \mathrm{~A}-\mathrm{H} 25 \mathrm{D}$ & 0.9800 \\
\hline $\mathrm{C} 20-\mathrm{C} 21$ & $1.608(15)$ & $\mathrm{C} 25 \mathrm{~A}-\mathrm{H} 25 \mathrm{E}$ & 0.9800 \\
\hline $\mathrm{C} 20-\mathrm{H} 20 \mathrm{~A}$ & 0.9900 & $\mathrm{C} 25 \mathrm{~A}-\mathrm{H} 25 \mathrm{~F}$ & 0.9800 \\
\hline $\mathrm{O} 1-\mathrm{Mo} 1-\mathrm{O} 2^{\mathrm{i}}$ & $74.40(14)$ & $\mathrm{C} 20-\mathrm{C} 21-\mathrm{H} 21 \mathrm{~A}$ & 109.5 \\
\hline $\mathrm{O} 1-\mathrm{Mo1}-\mathrm{S} 3$ & $132.28(12)$ & $\mathrm{C} 20-\mathrm{C} 21-\mathrm{H} 21 \mathrm{~B}$ & 109.5 \\
\hline $\mathrm{O} 22^{\mathrm{i}}-\mathrm{Mo} 1-\mathrm{S} 3$ & $83.24(11)$ & $\mathrm{H} 21 \mathrm{~A}-\mathrm{C} 21-\mathrm{H} 21 \mathrm{~B}$ & 109.5 \\
\hline $\mathrm{O} 1-\mathrm{Mo} 1-\mathrm{S} 1$ & $84.60(12)$ & $\mathrm{C} 20-\mathrm{C} 21-\mathrm{H} 21 \mathrm{C}$ & 109.5 \\
\hline $\mathrm{O} 2 \mathrm{i}-\mathrm{Mo} 1-\mathrm{S} 1$ & $133.48(12)$ & $\mathrm{H} 21 \mathrm{~A}-\mathrm{C} 21-\mathrm{H} 21 \mathrm{C}$ & 109.5 \\
\hline S3-Mo1-S1 & $137.72(6)$ & $\mathrm{H} 21 \mathrm{~B}-\mathrm{C} 21-\mathrm{H} 21 \mathrm{C}$ & 109.5 \\
\hline $\mathrm{O} 1-\mathrm{Mo} 1-\mathrm{S} 4$ & $83.76(11)$ & $\mathrm{C} 23-\mathrm{C} 22-\mathrm{N} 1$ & $116.1(11)$ \\
\hline $\mathrm{O} 2 \mathrm{i}-\mathrm{Mo} 1-\mathrm{S} 4$ & $133.74(12)$ & $\mathrm{C} 23-\mathrm{C} 22-\mathrm{H} 22 \mathrm{~A}$ & 108.3 \\
\hline $\mathrm{S} 3-\mathrm{Mo} 1-\mathrm{S} 4$ & $82.25(6)$ & $\mathrm{N} 1-\mathrm{C} 22-\mathrm{H} 22 \mathrm{~A}$ & 108.3 \\
\hline $\mathrm{S} 1-\mathrm{Mo1}-\mathrm{S} 4$ & $82.60(6)$ & $\mathrm{C} 23-\mathrm{C} 22-\mathrm{H} 22 \mathrm{~B}$ & 108.3 \\
\hline $\mathrm{O} 1-\mathrm{Mo} 1-\mathrm{S} 2$ & $133.64(11)$ & $\mathrm{N} 1-\mathrm{C} 22-\mathrm{H} 22 \mathrm{~B}$ & 108.3 \\
\hline $\mathrm{O} 2 \mathrm{i}-\mathrm{Mo} 1-\mathrm{S} 2$ & $83.25(11)$ & $\mathrm{H} 22 \mathrm{~A}-\mathrm{C} 22-\mathrm{H} 22 \mathrm{~B}$ & 107.4 \\
\hline $\mathrm{S} 3-\mathrm{Mo} 1-\mathrm{S} 2$ & $82.88(6)$ & $\mathrm{C} 22-\mathrm{C} 23-\mathrm{C} 24$ & $115.3(12)$ \\
\hline $\mathrm{S} 1-\mathrm{Mo} 1-\mathrm{S} 2$ & $82.21(7)$ & $\mathrm{C} 22-\mathrm{C} 23-\mathrm{H} 23 \mathrm{~A}$ & 108.4 \\
\hline $\mathrm{S} 4-\mathrm{Mo1}-\mathrm{S} 2$ & $137.48(6)$ & $\mathrm{C} 24-\mathrm{C} 23-\mathrm{H} 23 \mathrm{~A}$ & 108.4 \\
\hline $\mathrm{C} 1-\mathrm{S} 1-\mathrm{Mo} 1$ & $108.8(3)$ & $\mathrm{C} 22-\mathrm{C} 23-\mathrm{H} 23 \mathrm{~B}$ & 108.4 \\
\hline $\mathrm{C} 2-\mathrm{S} 2-\mathrm{Mo} 1$ & $109.4(2)$ & $\mathrm{C} 24-\mathrm{C} 23-\mathrm{H} 23 \mathrm{~B}$ & 108.4 \\
\hline $\mathrm{C} 5-\mathrm{S} 3-\mathrm{Mo} 1$ & $109.9(2)$ & $\mathrm{H} 23 \mathrm{~A}-\mathrm{C} 23-\mathrm{H} 23 \mathrm{~B}$ & 107.5 \\
\hline
\end{tabular}




\begin{tabular}{|c|c|}
\hline $\mathrm{C} 6-\mathrm{S} 4-\mathrm{Mo1}$ & $109.2(2)$ \\
\hline $\mathrm{C} 9-\mathrm{O} 1-\mathrm{Mo} 1$ & $116.0(3)$ \\
\hline $\mathrm{C} 9-\mathrm{O} 2-\mathrm{Mo}^{\mathrm{i}}$ & $117.0(3)$ \\
\hline $\mathrm{C} 2-\mathrm{C} 1-\mathrm{C} 3$ & $125.5(7)$ \\
\hline $\mathrm{C} 2-\mathrm{C} 1-\mathrm{S} 1$ & $120.3(6)$ \\
\hline $\mathrm{C} 3-\mathrm{C} 1-\mathrm{S} 1$ & $114.2(6)$ \\
\hline $\mathrm{C} 1-\mathrm{C} 2-\mathrm{C} 4$ & $126.2(8)$ \\
\hline $\mathrm{C} 1-\mathrm{C} 2-\mathrm{S} 2$ & $119.1(5)$ \\
\hline $\mathrm{C} 4-\mathrm{C} 2-\mathrm{S} 2$ & $114.6(7)$ \\
\hline $\mathrm{F} 3-\mathrm{C} 3-\mathrm{F} 2$ & $107.7(8)$ \\
\hline $\mathrm{F} 3-\mathrm{C} 3-\mathrm{F} 1$ & $106.8(7)$ \\
\hline $\mathrm{F} 2-\mathrm{C} 3-\mathrm{F} 1$ & $106.9(9)$ \\
\hline $\mathrm{F} 3-\mathrm{C} 3-\mathrm{C} 1$ & $112.3(9)$ \\
\hline $\mathrm{F} 2-\mathrm{C} 3-\mathrm{C} 1$ & $111.8(7)$ \\
\hline $\mathrm{F} 1-\mathrm{C} 3-\mathrm{C} 1$ & $111.0(7)$ \\
\hline $\mathrm{F} 4-\mathrm{C} 4-\mathrm{F} 6$ & $108.0(8)$ \\
\hline $\mathrm{F} 4-\mathrm{C} 4-\mathrm{F} 5$ & $108.8(9)$ \\
\hline $\mathrm{F} 6-\mathrm{C} 4-\mathrm{F} 5$ & $102.0(7)$ \\
\hline $\mathrm{F} 4-\mathrm{C} 4-\mathrm{C} 2$ & $112.7(7)$ \\
\hline $\mathrm{F} 6-\mathrm{C} 4-\mathrm{C} 2$ & $113.6(8)$ \\
\hline $\mathrm{F} 5-\mathrm{C} 4-\mathrm{C} 2$ & $111.2(8)$ \\
\hline $\mathrm{C} 6-\mathrm{C} 5-\mathrm{C} 7$ & $126.5(6)$ \\
\hline $\mathrm{C} 6-\mathrm{C} 5-\mathrm{S} 3$ & $119.1(5)$ \\
\hline $\mathrm{C} 7-\mathrm{C} 5-\mathrm{S} 3$ & $114.5(5)$ \\
\hline $\mathrm{C} 5-\mathrm{C} 6-\mathrm{C} 8$ & $126.4(7)$ \\
\hline $\mathrm{C} 5-\mathrm{C} 6-\mathrm{S} 4$ & $119.4(5)$ \\
\hline $\mathrm{C} 8-\mathrm{C} 6-\mathrm{S} 4$ & $114.2(5)$ \\
\hline $\mathrm{F} 9-\mathrm{C} 7-\mathrm{F} 7$ & $110.1(8)$ \\
\hline $\mathrm{F} 9-\mathrm{C} 7-\mathrm{F} 8$ & $105.1(7)$ \\
\hline $\mathrm{F} 7-\mathrm{C} 7-\mathrm{F} 8$ & $103.7(7)$ \\
\hline $\mathrm{F} 9-\mathrm{C} 7-\mathrm{C} 5$ & $113.1(6)$ \\
\hline $\mathrm{F} 7-\mathrm{C} 7-\mathrm{C} 5$ & $111.9(7)$ \\
\hline $\mathrm{F} 8-\mathrm{C} 7-\mathrm{C} 5$ & $112.3(7)$ \\
\hline $\mathrm{F} 12-\mathrm{C} 8-\mathrm{F} 11$ & $106.8(8)$ \\
\hline $\mathrm{F} 12-\mathrm{C} 8-\mathrm{F} 10$ & $107.5(7)$ \\
\hline $\mathrm{F} 11-\mathrm{C} 8-\mathrm{F} 10$ & $102.8(7)$ \\
\hline $\mathrm{F} 12-\mathrm{C} 8-\mathrm{C} 6$ & $113.4(6)$ \\
\hline $\mathrm{F} 11-\mathrm{C} 8-\mathrm{C} 6$ & $113.3(6)$ \\
\hline $\mathrm{F} 10-\mathrm{C} 8-\mathrm{C} 6$ & $112.4(7)$ \\
\hline $\mathrm{O} 1-\mathrm{C} 9-\mathrm{O} 2$ & $127.5(5)$ \\
\hline $\mathrm{O} 1-\mathrm{C} 9-\mathrm{C} 9^{\mathrm{i}}$ & $117.3(6)$ \\
\hline $\mathrm{O} 2-\mathrm{C} 9-\mathrm{C} 9^{\mathrm{i}}$ & $115.3(6)$ \\
\hline $\mathrm{C} 14-\mathrm{N} 1-\mathrm{C} 18$ & $113.4(12)$ \\
\hline $\mathrm{C} 14-\mathrm{N} 1-\mathrm{C} 10$ & $107.7(10)$ \\
\hline $\mathrm{C} 18-\mathrm{N} 1-\mathrm{C} 10$ & $109.6(10)$ \\
\hline $\mathrm{C} 14-\mathrm{N} 1-\mathrm{C} 22$ & $110.5(11)$ \\
\hline $\mathrm{C} 18-\mathrm{N} 1-\mathrm{C} 22$ & $107.6(10)$ \\
\hline $\mathrm{C} 10-\mathrm{N} 1-\mathrm{C} 22$ & $107.9(11)$ \\
\hline
\end{tabular}

\begin{tabular}{|c|c|}
\hline $\mathrm{C} 25-\mathrm{C} 24-\mathrm{C} 23$ & $111.7(19)$ \\
\hline $\mathrm{C} 25-\mathrm{C} 24-\mathrm{H} 24 \mathrm{~A}$ & 109.3 \\
\hline $\mathrm{C} 23-\mathrm{C} 24-\mathrm{H} 24 \mathrm{~A}$ & 109.3 \\
\hline $\mathrm{C} 25-\mathrm{C} 24-\mathrm{H} 24 \mathrm{~B}$ & 109.3 \\
\hline $\mathrm{C} 23-\mathrm{C} 24-\mathrm{H} 24 \mathrm{~B}$ & 109.3 \\
\hline $\mathrm{H} 24 \mathrm{~A}-\mathrm{C} 24-\mathrm{H} 24 \mathrm{~B}$ & 107.9 \\
\hline $\mathrm{C} 24-\mathrm{C} 25-\mathrm{H} 25 \mathrm{~A}$ & 109.5 \\
\hline $\mathrm{C} 24-\mathrm{C} 25-\mathrm{H} 25 \mathrm{~B}$ & 109.5 \\
\hline $\mathrm{H} 25 \mathrm{~A}-\mathrm{C} 25-\mathrm{H} 25 \mathrm{~B}$ & 109.5 \\
\hline $\mathrm{C} 24-\mathrm{C} 25-\mathrm{H} 25 \mathrm{C}$ & 109.5 \\
\hline $\mathrm{H} 25 \mathrm{~A}-\mathrm{C} 25-\mathrm{H} 25 \mathrm{C}$ & 109.5 \\
\hline $\mathrm{H} 25 \mathrm{~B}-\mathrm{C} 25-\mathrm{H} 25 \mathrm{C}$ & 109.5 \\
\hline $\mathrm{C} 14 \mathrm{~A}-\mathrm{N} 1 \mathrm{~A}-\mathrm{C} 10 \mathrm{~A}$ & $103.8(13)$ \\
\hline $\mathrm{C} 14 \mathrm{~A}-\mathrm{N} 1 \mathrm{~A}-\mathrm{C} 22 \mathrm{~A}$ & $112.4(16)$ \\
\hline $\mathrm{C} 10 \mathrm{~A}-\mathrm{N} 1 \mathrm{~A}-\mathrm{C} 22 \mathrm{~A}$ & $112.7(16)$ \\
\hline $\mathrm{C} 14 \mathrm{~A}-\mathrm{N} 1 \mathrm{~A}-\mathrm{C} 18 \mathrm{~A}$ & $111.1(15)$ \\
\hline $\mathrm{C} 10 \mathrm{~A}-\mathrm{N} 1 \mathrm{~A}-\mathrm{C} 18 \mathrm{~A}$ & $112.3(14)$ \\
\hline $\mathrm{C} 22 \mathrm{~A}-\mathrm{N} 1 \mathrm{~A}-\mathrm{C} 18 \mathrm{~A}$ & $104.6(14)$ \\
\hline $\mathrm{C} 11 \mathrm{~A}-\mathrm{C} 10 \mathrm{~A}-\mathrm{N} 1 \mathrm{~A}$ & $115.9(14)$ \\
\hline $\mathrm{C} 11 \mathrm{~A}-\mathrm{C} 10 \mathrm{~A}-\mathrm{H} 10 \mathrm{C}$ & 108.3 \\
\hline $\mathrm{N} 1 \mathrm{~A}-\mathrm{C} 10 \mathrm{~A}-\mathrm{H} 10 \mathrm{C}$ & 108.3 \\
\hline $\mathrm{C} 11 \mathrm{~A}-\mathrm{C} 10 \mathrm{~A}-\mathrm{H} 10 \mathrm{D}$ & 108.3 \\
\hline $\mathrm{N} 1 \mathrm{~A}-\mathrm{C} 10 \mathrm{~A}-\mathrm{H} 10 \mathrm{D}$ & 108.3 \\
\hline $\mathrm{H} 10 \mathrm{C}-\mathrm{C} 10 \mathrm{~A}-\mathrm{H} 10 \mathrm{D}$ & 107.4 \\
\hline $\mathrm{C} 10 \mathrm{~A}-\mathrm{C} 11 \mathrm{~A}-\mathrm{C} 12 \mathrm{~A}$ & $109.1(13)$ \\
\hline $\mathrm{C} 10 \mathrm{~A}-\mathrm{C} 11 \mathrm{~A}-\mathrm{H} 11 \mathrm{C}$ & 109.9 \\
\hline $\mathrm{C} 12 \mathrm{~A}-\mathrm{C} 11 \mathrm{~A}-\mathrm{H} 11 \mathrm{C}$ & 109.9 \\
\hline $\mathrm{C} 10 \mathrm{~A}-\mathrm{C} 11 \mathrm{~A}-\mathrm{H} 11 \mathrm{D}$ & 109.9 \\
\hline $\mathrm{C} 12 \mathrm{~A}-\mathrm{C} 11 \mathrm{~A}-\mathrm{H} 11 \mathrm{D}$ & 109.9 \\
\hline $\mathrm{H} 11 \mathrm{C}-\mathrm{C} 11 \mathrm{~A}-\mathrm{H} 11 \mathrm{D}$ & 108.3 \\
\hline $\mathrm{C} 13 \mathrm{~A}-\mathrm{C} 12 \mathrm{~A}-\mathrm{C} 11 \mathrm{~A}$ & $108.5(15)$ \\
\hline $\mathrm{C} 13 \mathrm{~A}-\mathrm{C} 12 \mathrm{~A}-\mathrm{H} 12 \mathrm{C}$ & 110.0 \\
\hline $\mathrm{C} 11 \mathrm{~A}-\mathrm{C} 12 \mathrm{~A}-\mathrm{H} 12 \mathrm{C}$ & 110.0 \\
\hline $\mathrm{C} 13 \mathrm{~A}-\mathrm{C} 12 \mathrm{~A}-\mathrm{H} 12 \mathrm{D}$ & 110.0 \\
\hline $\mathrm{C} 11 \mathrm{~A}-\mathrm{C} 12 \mathrm{~A}-\mathrm{H} 12 \mathrm{D}$ & 110.0 \\
\hline $\mathrm{H} 12 \mathrm{C}-\mathrm{C} 12 \mathrm{~A}-\mathrm{H} 12 \mathrm{D}$ & 108.4 \\
\hline $\mathrm{C} 12 \mathrm{~A}-\mathrm{C} 13 \mathrm{~A}-\mathrm{H} 13 \mathrm{D}$ & 109.5 \\
\hline $\mathrm{C} 12 \mathrm{~A}-\mathrm{C} 13 \mathrm{~A}-\mathrm{H} 13 \mathrm{E}$ & 109.5 \\
\hline $\mathrm{H} 13 \mathrm{D}-\mathrm{C} 13 \mathrm{~A}-\mathrm{H} 13 \mathrm{E}$ & 109.5 \\
\hline $\mathrm{C} 12 \mathrm{~A}-\mathrm{C} 13 \mathrm{~A}-\mathrm{H} 13 \mathrm{~F}$ & 109.5 \\
\hline $\mathrm{H} 13 \mathrm{D}-\mathrm{C} 13 \mathrm{~A}-\mathrm{H} 13 \mathrm{~F}$ & 109.5 \\
\hline $\mathrm{H} 13 \mathrm{E}-\mathrm{C} 13 \mathrm{~A}-\mathrm{H} 13 \mathrm{~F}$ & 109.5 \\
\hline $\mathrm{N} 1 \mathrm{~A}-\mathrm{C} 14 \mathrm{~A}-\mathrm{C} 15 \mathrm{~A}$ & $112.1(16)$ \\
\hline $\mathrm{N} 1 \mathrm{~A}-\mathrm{C} 14 \mathrm{~A}-\mathrm{H} 14 \mathrm{C}$ & 109.2 \\
\hline $\mathrm{C} 15 \mathrm{~A}-\mathrm{C} 14 \mathrm{~A}-\mathrm{H} 14 \mathrm{C}$ & 109.2 \\
\hline N1A-C14A-H14D & 109.2 \\
\hline $\mathrm{C} 15 \mathrm{~A}-\mathrm{C} 14 \mathrm{~A}-\mathrm{H} 14 \mathrm{D}$ & 109.2 \\
\hline $\mathrm{H} 14 \mathrm{C}-\mathrm{C} 14 \mathrm{~A}-\mathrm{H} 14 \mathrm{D}$ & 107.9 \\
\hline
\end{tabular}




\begin{tabular}{|c|c|c|c|}
\hline $\mathrm{C} 11-\mathrm{C} 10-\mathrm{N} 1$ & $116.8(11)$ & $\mathrm{C} 16 \mathrm{~A}-\mathrm{C} 15 \mathrm{~A}-\mathrm{C} 14 \mathrm{~A}$ & $113.8(16)$ \\
\hline $\mathrm{C} 11-\mathrm{C} 10-\mathrm{H} 10 \mathrm{~A}$ & 108.1 & $\mathrm{C} 16 \mathrm{~A}-\mathrm{C} 15 \mathrm{~A}-\mathrm{H} 15 \mathrm{C}$ & 108.8 \\
\hline $\mathrm{N} 1-\mathrm{C} 10-\mathrm{H} 10 \mathrm{~A}$ & 108.1 & $\mathrm{C} 14 \mathrm{~A}-\mathrm{C} 15 \mathrm{~A}-\mathrm{H} 15 \mathrm{C}$ & 108.8 \\
\hline $\mathrm{C} 11-\mathrm{C} 10-\mathrm{H} 10 \mathrm{~B}$ & 108.1 & $\mathrm{C} 16 \mathrm{~A}-\mathrm{C} 15 \mathrm{~A}-\mathrm{H} 15 \mathrm{D}$ & 108.8 \\
\hline $\mathrm{N} 1-\mathrm{C} 10-\mathrm{H} 10 \mathrm{~B}$ & 108.1 & $\mathrm{C} 14 \mathrm{~A}-\mathrm{C} 15 \mathrm{~A}-\mathrm{H} 15 \mathrm{D}$ & 108.8 \\
\hline $\mathrm{H} 10 \mathrm{~A}-\mathrm{C} 10-\mathrm{H} 10 \mathrm{~B}$ & 107.3 & $\mathrm{H} 15 \mathrm{C}-\mathrm{C} 15 \mathrm{~A}-\mathrm{H} 15 \mathrm{D}$ & 107.7 \\
\hline $\mathrm{C} 10-\mathrm{C} 11-\mathrm{C} 12$ & $111.2(11)$ & $\mathrm{C} 15 \mathrm{~A}-\mathrm{C} 16 \mathrm{~A}-\mathrm{C} 17 \mathrm{~A}$ & $118.9(18)$ \\
\hline $\mathrm{C} 10-\mathrm{C} 11-\mathrm{H} 11 \mathrm{~A}$ & 109.4 & $\mathrm{C} 15 \mathrm{~A}-\mathrm{C} 16 \mathrm{~A}-\mathrm{H} 16 \mathrm{C}$ & 107.6 \\
\hline $\mathrm{C} 12-\mathrm{C} 11-\mathrm{H} 11 \mathrm{~A}$ & 109.4 & $\mathrm{C} 17 \mathrm{~A}-\mathrm{C} 16 \mathrm{~A}-\mathrm{H} 16 \mathrm{C}$ & 107.6 \\
\hline $\mathrm{C} 10-\mathrm{C} 11-\mathrm{H} 11 \mathrm{~B}$ & 109.4 & $\mathrm{C} 15 \mathrm{~A}-\mathrm{C} 16 \mathrm{~A}-\mathrm{H} 16 \mathrm{D}$ & 107.6 \\
\hline $\mathrm{C} 12-\mathrm{C} 11-\mathrm{H} 11 \mathrm{~B}$ & 109.4 & $\mathrm{C} 17 \mathrm{~A}-\mathrm{C} 16 \mathrm{~A}-\mathrm{H} 16 \mathrm{D}$ & 107.6 \\
\hline $\mathrm{H} 11 \mathrm{~A}-\mathrm{C} 11-\mathrm{H} 11 \mathrm{~B}$ & 108.0 & $\mathrm{H} 16 \mathrm{C}-\mathrm{C} 16 \mathrm{~A}-\mathrm{H} 16 \mathrm{D}$ & 107.0 \\
\hline $\mathrm{C} 13-\mathrm{C} 12-\mathrm{C} 11$ & $109.5(13)$ & $\mathrm{C} 16 \mathrm{~A}-\mathrm{C} 17 \mathrm{~A}-\mathrm{H} 17 \mathrm{D}$ & 109.5 \\
\hline $\mathrm{C} 13-\mathrm{C} 12-\mathrm{H} 12 \mathrm{~A}$ & 109.8 & $\mathrm{C} 16 \mathrm{~A}-\mathrm{C} 17 \mathrm{~A}-\mathrm{H} 17 \mathrm{E}$ & 109.5 \\
\hline $\mathrm{C} 11-\mathrm{C} 12-\mathrm{H} 12 \mathrm{~A}$ & 109.8 & $\mathrm{H} 17 \mathrm{D}-\mathrm{C} 17 \mathrm{~A}-\mathrm{H} 17 \mathrm{E}$ & 109.5 \\
\hline $\mathrm{C} 13-\mathrm{C} 12-\mathrm{H} 12 \mathrm{~B}$ & 109.8 & $\mathrm{C} 16 \mathrm{~A}-\mathrm{C} 17 \mathrm{~A}-\mathrm{H} 17 \mathrm{~F}$ & 109.5 \\
\hline $\mathrm{C} 11-\mathrm{C} 12-\mathrm{H} 12 \mathrm{~B}$ & 109.8 & $\mathrm{H} 17 \mathrm{D}-\mathrm{C} 17 \mathrm{~A}-\mathrm{H} 17 \mathrm{~F}$ & 109.5 \\
\hline $\mathrm{H} 12 \mathrm{~A}-\mathrm{C} 12-\mathrm{H} 12 \mathrm{~B}$ & 108.2 & $\mathrm{H} 17 \mathrm{E}-\mathrm{C} 17 \mathrm{~A}-\mathrm{H} 17 \mathrm{~F}$ & 109.5 \\
\hline $\mathrm{C} 12-\mathrm{C} 13-\mathrm{H} 13 \mathrm{~A}$ & 109.5 & $\mathrm{~N} 1 \mathrm{~A}-\mathrm{C} 18 \mathrm{~A}-\mathrm{C} 19 \mathrm{~A}$ & $109.4(14)$ \\
\hline $\mathrm{C} 12-\mathrm{C} 13-\mathrm{H} 13 \mathrm{~B}$ & 109.5 & $\mathrm{~N} 1 \mathrm{~A}-\mathrm{C} 18 \mathrm{~A}-\mathrm{H} 18 \mathrm{C}$ & 109.8 \\
\hline $\mathrm{H} 13 \mathrm{~A}-\mathrm{C} 13-\mathrm{H} 13 \mathrm{~B}$ & 109.5 & $\mathrm{C} 19 \mathrm{~A}-\mathrm{C} 18 \mathrm{~A}-\mathrm{H} 18 \mathrm{C}$ & 109.8 \\
\hline $\mathrm{C} 12-\mathrm{C} 13-\mathrm{H} 13 \mathrm{C}$ & 109.5 & $\mathrm{~N} 1 \mathrm{~A}-\mathrm{C} 18 \mathrm{~A}-\mathrm{H} 18 \mathrm{D}$ & 109.8 \\
\hline $\mathrm{H} 13 \mathrm{~A}-\mathrm{C} 13-\mathrm{H} 13 \mathrm{C}$ & 109.5 & $\mathrm{C} 19 \mathrm{~A}-\mathrm{C} 18 \mathrm{~A}-\mathrm{H} 18 \mathrm{D}$ & 109.8 \\
\hline $\mathrm{H} 13 \mathrm{~B}-\mathrm{C} 13-\mathrm{H} 13 \mathrm{C}$ & 109.5 & $\mathrm{H} 18 \mathrm{C}-\mathrm{C} 18 \mathrm{~A}-\mathrm{H} 18 \mathrm{D}$ & 108.2 \\
\hline $\mathrm{N} 1-\mathrm{C} 14-\mathrm{C} 15$ & $116.8(13)$ & $\mathrm{C} 20 \mathrm{~A}-\mathrm{C} 19 \mathrm{~A}-\mathrm{C} 18 \mathrm{~A}$ & $110.4(14)$ \\
\hline $\mathrm{N} 1-\mathrm{C} 14-\mathrm{H} 14 \mathrm{~A}$ & 108.1 & $\mathrm{C} 20 \mathrm{~A}-\mathrm{C} 19 \mathrm{~A}-\mathrm{H} 19 \mathrm{C}$ & 109.6 \\
\hline $\mathrm{C} 15-\mathrm{C} 14-\mathrm{H} 14 \mathrm{~A}$ & 108.1 & $\mathrm{C} 18 \mathrm{~A}-\mathrm{C} 19 \mathrm{~A}-\mathrm{H} 19 \mathrm{C}$ & 109.6 \\
\hline $\mathrm{N} 1-\mathrm{C} 14-\mathrm{H} 14 \mathrm{~B}$ & 108.1 & $\mathrm{C} 20 \mathrm{~A}-\mathrm{C} 19 \mathrm{~A}-\mathrm{H} 19 \mathrm{D}$ & 109.6 \\
\hline $\mathrm{C} 15-\mathrm{C} 14-\mathrm{H} 14 \mathrm{~B}$ & 108.1 & $\mathrm{C} 18 \mathrm{~A}-\mathrm{C} 19 \mathrm{~A}-\mathrm{H} 19 \mathrm{D}$ & 109.6 \\
\hline $\mathrm{H} 14 \mathrm{~A}-\mathrm{C} 14-\mathrm{H} 14 \mathrm{~B}$ & 107.3 & $\mathrm{H} 19 \mathrm{C}-\mathrm{C} 19 \mathrm{~A}-\mathrm{H} 19 \mathrm{D}$ & 108.1 \\
\hline $\mathrm{C} 16-\mathrm{C} 15-\mathrm{C} 14$ & $112.3(11)$ & $\mathrm{C} 19 \mathrm{~A}-\mathrm{C} 20 \mathrm{~A}-\mathrm{C} 21 \mathrm{~A}$ & $107.4(16)$ \\
\hline $\mathrm{C} 16-\mathrm{C} 15-\mathrm{H} 15 \mathrm{~A}$ & 109.1 & $\mathrm{C} 19 \mathrm{~A}-\mathrm{C} 20 \mathrm{~A}-\mathrm{H} 20 \mathrm{C}$ & 110.2 \\
\hline $\mathrm{C} 14-\mathrm{C} 15-\mathrm{H} 15 \mathrm{~A}$ & 109.1 & $\mathrm{C} 21 \mathrm{~A}-\mathrm{C} 20 \mathrm{~A}-\mathrm{H} 20 \mathrm{C}$ & 110.2 \\
\hline $\mathrm{C} 16-\mathrm{C} 15-\mathrm{H} 15 \mathrm{~B}$ & 109.1 & $\mathrm{C} 19 \mathrm{~A}-\mathrm{C} 20 \mathrm{~A}-\mathrm{H} 20 \mathrm{D}$ & 110.2 \\
\hline $\mathrm{C} 14-\mathrm{C} 15-\mathrm{H} 15 \mathrm{~B}$ & 109.1 & $\mathrm{C} 21 \mathrm{~A}-\mathrm{C} 20 \mathrm{~A}-\mathrm{H} 20 \mathrm{D}$ & 110.2 \\
\hline $\mathrm{H} 15 \mathrm{~A}-\mathrm{C} 15-\mathrm{H} 15 \mathrm{~B}$ & 107.9 & $\mathrm{H} 20 \mathrm{C}-\mathrm{C} 20 \mathrm{~A}-\mathrm{H} 20 \mathrm{D}$ & 108.5 \\
\hline $\mathrm{C} 15-\mathrm{C} 16-\mathrm{C} 17$ & $108.5(12)$ & $\mathrm{C} 20 \mathrm{~A}-\mathrm{C} 21 \mathrm{~A}-\mathrm{H} 21 \mathrm{D}$ & 109.5 \\
\hline $\mathrm{C} 15-\mathrm{C} 16-\mathrm{H} 16 \mathrm{~A}$ & 110.0 & $\mathrm{C} 20 \mathrm{~A}-\mathrm{C} 21 \mathrm{~A}-\mathrm{H} 21 \mathrm{E}$ & 109.5 \\
\hline $\mathrm{C} 17-\mathrm{C} 16-\mathrm{H} 16 \mathrm{~A}$ & 110.0 & $\mathrm{H} 21 \mathrm{D}-\mathrm{C} 21 \mathrm{~A}-\mathrm{H} 21 \mathrm{E}$ & 109.5 \\
\hline $\mathrm{C} 15-\mathrm{C} 16-\mathrm{H} 16 \mathrm{~B}$ & 110.0 & $\mathrm{C} 20 \mathrm{~A}-\mathrm{C} 21 \mathrm{~A}-\mathrm{H} 21 \mathrm{~F}$ & 109.5 \\
\hline $\mathrm{C} 17-\mathrm{C} 16-\mathrm{H} 16 \mathrm{~B}$ & 110.0 & $\mathrm{H} 21 \mathrm{D}-\mathrm{C} 21 \mathrm{~A}-\mathrm{H} 21 \mathrm{~F}$ & 109.5 \\
\hline $\mathrm{H} 16 \mathrm{~A}-\mathrm{C} 16-\mathrm{H} 16 \mathrm{~B}$ & 108.4 & $\mathrm{H} 21 \mathrm{E}-\mathrm{C} 21 \mathrm{~A}-\mathrm{H} 21 \mathrm{~F}$ & 109.5 \\
\hline $\mathrm{C} 16-\mathrm{C} 17-\mathrm{H} 17 \mathrm{~A}$ & 109.5 & $\mathrm{C} 23 \mathrm{~A}-\mathrm{C} 22 \mathrm{~A}-\mathrm{N} 1 \mathrm{~A}$ & $118.1(16)$ \\
\hline $\mathrm{C} 16-\mathrm{C} 17-\mathrm{H} 17 \mathrm{~B}$ & 109.5 & $\mathrm{C} 23 \mathrm{~A}-\mathrm{C} 22 \mathrm{~A}-\mathrm{H} 22 \mathrm{C}$ & 107.8 \\
\hline $\mathrm{H} 17 \mathrm{~A}-\mathrm{C} 17-\mathrm{H} 17 \mathrm{~B}$ & 109.5 & $\mathrm{~N} 1 \mathrm{~A}-\mathrm{C} 22 \mathrm{~A}-\mathrm{H} 22 \mathrm{C}$ & 107.8 \\
\hline $\mathrm{C} 16-\mathrm{C} 17-\mathrm{H} 17 \mathrm{C}$ & 109.5 & $\mathrm{C} 23 \mathrm{~A}-\mathrm{C} 22 \mathrm{~A}-\mathrm{H} 22 \mathrm{D}$ & 107.8 \\
\hline $\mathrm{H} 17 \mathrm{~A}-\mathrm{C} 17-\mathrm{H} 17 \mathrm{C}$ & 109.5 & $\mathrm{~N} 1 \mathrm{~A}-\mathrm{C} 22 \mathrm{~A}-\mathrm{H} 22 \mathrm{D}$ & 107.8 \\
\hline $\mathrm{H} 17 \mathrm{~B}-\mathrm{C} 17-\mathrm{H} 17 \mathrm{C}$ & 109.5 & $\mathrm{H} 22 \mathrm{C}-\mathrm{C} 22 \mathrm{~A}-\mathrm{H} 22 \mathrm{D}$ & 107.1 \\
\hline
\end{tabular}




$\begin{array}{ll}\text { N1-C18-C19 } & 113.4(11) \\ \text { N1-C18-H18A } & 108.9 \\ \text { C19-C18-H18A } & 108.9 \\ \text { N1-C18-H18B } & 108.9 \\ \text { C19-C18-H18B } & 108.9 \\ \text { H18A-C18-H18B } & 107.7 \\ \text { C20-C19-C18 } & 109.6(11) \\ \text { C20-C19-H19A } & 109.7 \\ \text { C18-C19-H19A } & 109.7 \\ \text { C20-C19-H19B } & 109.7 \\ \text { C18-C19-H19B } & 109.7 \\ \text { H19A-C19-H19B } & 108.2 \\ \text { C19-C20-C21 } & 110.4(11) \\ \text { C19-C20-H20A } & 109.6 \\ \text { C21-C20-H20A } & 109.6 \\ \text { C19-C20-H20B } & 109.6 \\ \text { C21-C20-H20B } & 109.6 \\ \text { H20A-C20-H20B } & 108.1 \\ \text { Mo1-S1-C1-C2 } & \\ \text { Mo1-S1-C1-C3 } & -3.8(6) \\ \text { C3-C1-C2-C4 } & 179.4(5) \\ \text { S1-C1-C2-C4 } & -4.0(12) \\ \text { C3-C1-C2-S2 } & 179.7(6) \\ \text { S1-C1-C2-S2 } & 177.6(6) \\ \text { Mo1-S2-C2-C1 } & 1.3(8) \\ \text { Mo1-S2-C2-C4 } & 1.9(6) \\ \text { C2-C1-C3-F3 } & -176.6(5) \\ \text { S1-C1-C3-F3 } & 45.3(11) \\ \text { C2-C1-C3-F2 } & -138.2(6) \\ \text { S1-C1-C3-F2 } & -76.0(11) \\ \text { C2-C1-C3-F1 } & 100.6(8) \\ \text { S1-C1-C3-F1 } & 164.8(8) \\ \text { C1-C2-C4-F4 } & -18.6(10) \\ \text { S2-C2-C4-F4 } & -84.2(12) \\ \text { C1-C2-C4-F6 } & 94.3(10) \\ \text { S2-C2-C4-F6 } & 39.0(11) \\ \text { C1-C2-C4-F5 } & -142.6(7) \\ \text { S2-C2-C4-F5 } & 153.4(8) \\ \text { Mo1-S3-C5-C6 } & -28.2(9) \\ \text { Mo1-S3-C5-C7 } & -3.1(6) \\ \text { C7-C5-C6-C8 } & 176.5(4) \\ \text { S3-C5-C6-C8 } & 2.0(11) \\ \text { C7-C5-C6-S4 } & -178.4(6) \\ \text { S3-C5-C6-S4 } & -179.3(5) \\ \text { C6-C5-C7-F9 } 1-S 4-C 6-C 5 & \\ & \end{array}$

\begin{tabular}{|c|c|}
\hline $\mathrm{C} 22 \mathrm{~A}-\mathrm{C} 23 \mathrm{~A}-\mathrm{C} 24 \mathrm{~A}$ & $108.6(16)$ \\
\hline $\mathrm{C} 22 \mathrm{~A}-\mathrm{C} 23 \mathrm{~A}-\mathrm{H} 23 \mathrm{C}$ & 110.0 \\
\hline $\mathrm{C} 24 \mathrm{~A}-\mathrm{C} 23 \mathrm{~A}-\mathrm{H} 23 \mathrm{C}$ & 110.0 \\
\hline $\mathrm{C} 22 \mathrm{~A}-\mathrm{C} 23 \mathrm{~A}-\mathrm{H} 23 \mathrm{D}$ & 110.0 \\
\hline $\mathrm{C} 24 \mathrm{~A}-\mathrm{C} 23 \mathrm{~A}-\mathrm{H} 23 \mathrm{D}$ & 110.0 \\
\hline $\mathrm{H} 23 \mathrm{C}-\mathrm{C} 23 \mathrm{~A}-\mathrm{H} 23 \mathrm{D}$ & 108.4 \\
\hline $\mathrm{C} 25 \mathrm{~A}-\mathrm{C} 24 \mathrm{~A}-\mathrm{C} 23 \mathrm{~A}$ & $110.4(17)$ \\
\hline $\mathrm{C} 25 \mathrm{~A}-\mathrm{C} 24 \mathrm{~A}-\mathrm{H} 24 \mathrm{C}$ & 109.6 \\
\hline $\mathrm{C} 23 \mathrm{~A}-\mathrm{C} 24 \mathrm{~A}-\mathrm{H} 24 \mathrm{C}$ & 109.6 \\
\hline $\mathrm{C} 25 \mathrm{~A}-\mathrm{C} 24 \mathrm{~A}-\mathrm{H} 24 \mathrm{D}$ & 109.6 \\
\hline $\mathrm{C} 23 \mathrm{~A}-\mathrm{C} 24 \mathrm{~A}-\mathrm{H} 24 \mathrm{D}$ & 109.6 \\
\hline $\mathrm{H} 24 \mathrm{C}-\mathrm{C} 24 \mathrm{~A}-\mathrm{H} 24 \mathrm{D}$ & 108.1 \\
\hline $\mathrm{C} 24 \mathrm{~A}-\mathrm{C} 25 \mathrm{~A}-\mathrm{H} 25 \mathrm{D}$ & 109.5 \\
\hline $\mathrm{C} 24 \mathrm{~A}-\mathrm{C} 25 \mathrm{~A}-\mathrm{H} 25 \mathrm{E}$ & 109.5 \\
\hline $\mathrm{H} 25 \mathrm{D}-\mathrm{C} 25 \mathrm{~A}-\mathrm{H} 25 \mathrm{E}$ & 109.5 \\
\hline $\mathrm{C} 24 \mathrm{~A}-\mathrm{C} 25 \mathrm{~A}-\mathrm{H} 25 \mathrm{~F}$ & 109.5 \\
\hline $\mathrm{H} 25 \mathrm{D}-\mathrm{C} 25 \mathrm{~A}-\mathrm{H} 25 \mathrm{~F}$ & 109.5 \\
\hline $\mathrm{H} 25 \mathrm{E}-\mathrm{C} 25 \mathrm{~A}-\mathrm{H} 25 \mathrm{~F}$ & 109.5 \\
\hline $\mathrm{Mo1}-\mathrm{O} 2-\mathrm{C} 9-\mathrm{O} 1$ & $179.0(5)$ \\
\hline $\mathrm{Mo} 1-\mathrm{O} 2-\mathrm{C} 9-\mathrm{C} 9^{\mathrm{i}}$ & $-1.7(8)$ \\
\hline $\mathrm{C} 14-\mathrm{N} 1-\mathrm{C} 10-\mathrm{C} 11$ & $-172.6(18)$ \\
\hline $\mathrm{C} 18-\mathrm{N} 1-\mathrm{C} 10-\mathrm{C} 11$ & $63.6(18)$ \\
\hline $\mathrm{C} 22-\mathrm{N} 1-\mathrm{C} 10-\mathrm{C} 11$ & $-53.3(18)$ \\
\hline $\mathrm{N} 1-\mathrm{C} 10-\mathrm{C} 11-\mathrm{C} 12$ & $-172.9(13)$ \\
\hline $\mathrm{C} 10-\mathrm{C} 11-\mathrm{C} 12-\mathrm{C} 13$ & $89(2)$ \\
\hline $\mathrm{C} 18-\mathrm{N} 1-\mathrm{C} 14-\mathrm{C} 15$ & $-74(3)$ \\
\hline $\mathrm{C} 10-\mathrm{N} 1-\mathrm{C} 14-\mathrm{C} 15$ & $165(2)$ \\
\hline $\mathrm{C} 22-\mathrm{N} 1-\mathrm{C} 14-\mathrm{C} 15$ & $47(3)$ \\
\hline $\mathrm{N} 1-\mathrm{C} 14-\mathrm{C} 15-\mathrm{C} 16$ & $-177.5(16)$ \\
\hline $\mathrm{C} 14-\mathrm{C} 15-\mathrm{C} 16-\mathrm{C} 17$ & $-65(2)$ \\
\hline $\mathrm{C} 14-\mathrm{N} 1-\mathrm{C} 18-\mathrm{C} 19$ & $-38.5(19)$ \\
\hline $\mathrm{C} 10-\mathrm{N} 1-\mathrm{C} 18-\mathrm{C} 19$ & $81.9(17)$ \\
\hline $\mathrm{C} 22-\mathrm{N} 1-\mathrm{C} 18-\mathrm{C} 19$ & $-161.0(17)$ \\
\hline $\mathrm{N} 1-\mathrm{C} 18-\mathrm{C} 19-\mathrm{C} 20$ & $176.0(12)$ \\
\hline $\mathrm{C} 18-\mathrm{C} 19-\mathrm{C} 20-\mathrm{C} 21$ & $137.9(18)$ \\
\hline $\mathrm{C} 14-\mathrm{N} 1-\mathrm{C} 22-\mathrm{C} 23$ & $61(3)$ \\
\hline $\mathrm{C} 18-\mathrm{N} 1-\mathrm{C} 22-\mathrm{C} 23$ & $-174(2)$ \\
\hline $\mathrm{C} 10-\mathrm{N} 1-\mathrm{C} 22-\mathrm{C} 23$ & $-56(3)$ \\
\hline $\mathrm{N} 1-\mathrm{C} 22-\mathrm{C} 23-\mathrm{C} 24$ & $175(2)$ \\
\hline $\mathrm{C} 22-\mathrm{C} 23-\mathrm{C} 24-\mathrm{C} 25$ & $68(3)$ \\
\hline $\mathrm{C} 14 \mathrm{~A}-\mathrm{N} 1 \mathrm{~A}-\mathrm{C} 10 \mathrm{~A}-\mathrm{C} 11 \mathrm{~A}$ & $177(3)$ \\
\hline $\mathrm{C} 22 \mathrm{~A}-\mathrm{N} 1 \mathrm{~A}-\mathrm{C} 10 \mathrm{~A}-\mathrm{C} 11 \mathrm{~A}$ & $-61(3)$ \\
\hline $\mathrm{C} 18 \mathrm{~A}-\mathrm{N} 1 \mathrm{~A}-\mathrm{C} 10 \mathrm{~A}-\mathrm{C} 11 \mathrm{~A}$ & $57(3)$ \\
\hline $\mathrm{N} 1 \mathrm{~A}-\mathrm{C} 10 \mathrm{~A}-\mathrm{C} 11 \mathrm{~A}-\mathrm{C} 12 \mathrm{~A}$ & $178(2)$ \\
\hline $\mathrm{C} 10 \mathrm{~A}-\mathrm{C} 11 \mathrm{~A}-\mathrm{C} 12 \mathrm{~A}-\mathrm{C} 13 \mathrm{~A}$ & $-161(2)$ \\
\hline $\mathrm{C} 10 \mathrm{~A}-\mathrm{N} 1 \mathrm{~A}-\mathrm{C} 14 \mathrm{~A}-\mathrm{C} 15 \mathrm{~A}$ & $-166(3)$ \\
\hline $\mathrm{C} 22 \mathrm{~A}-\mathrm{N} 1 \mathrm{~A}-\mathrm{C} 14 \mathrm{~A}-\mathrm{C} 15 \mathrm{~A}$ & $72(4)$ \\
\hline
\end{tabular}




$\begin{array}{llll}\mathrm{S} 3-\mathrm{C} 5-\mathrm{C} 7-\mathrm{F} 9 & -101.8(7) & \mathrm{C} 18 \mathrm{~A}-\mathrm{N} 1 \mathrm{~A}-\mathrm{C} 14 \mathrm{~A}-\mathrm{C} 15 \mathrm{~A} & -45(3) \\ \mathrm{C} 6-\mathrm{C} 5-\mathrm{C} 7-\mathrm{F} 7 & -157.1(8) & \mathrm{N} 1 \mathrm{~A}-\mathrm{C} 14 \mathrm{~A}-\mathrm{C} 15 \mathrm{~A}-\mathrm{C} 16 \mathrm{~A} & 160(2) \\ \mathrm{S} 3-\mathrm{C} 5-\mathrm{C} 7-\mathrm{F} 7 & 23.3(9) & \mathrm{C} 14 \mathrm{~A}-\mathrm{C} 15 \mathrm{~A}-\mathrm{C} 16 \mathrm{~A}-\mathrm{C} 17 \mathrm{~A} & 29(4) \\ \mathrm{C} 6-\mathrm{C} 5-\mathrm{C} 7-\mathrm{F} 8 & -40.9(10) & \mathrm{C} 14 \mathrm{~A}-\mathrm{N} 1 \mathrm{~A}-\mathrm{C} 18 \mathrm{~A}-\mathrm{C} 19 \mathrm{~A} & -85(3) \\ \mathrm{S} 3-\mathrm{C} 5-\mathrm{C} 7-\mathrm{F} 8 & 139.5(6) & \mathrm{C} 10 \mathrm{~A}-\mathrm{N} 1 \mathrm{~A}-\mathrm{C} 18 \mathrm{~A}-\mathrm{C} 19 \mathrm{~A} & 31(3) \\ \mathrm{C} 5-\mathrm{C} 6-\mathrm{C} 8-\mathrm{F} 12 & 83.2(10) & \mathrm{C} 22 \mathrm{~A}-\mathrm{N} 1 \mathrm{~A}-\mathrm{C} 18 \mathrm{~A}-\mathrm{C} 19 \mathrm{~A} & 153(3) \\ \text { S4-C6-C8-F12 } & -95.5(7) & \mathrm{N} 1 \mathrm{~A}-\mathrm{C} 18 \mathrm{~A}-\mathrm{C} 19 \mathrm{~A}-\mathrm{C} 20 \mathrm{~A} & -165.9(18) \\ \mathrm{C} 5-\mathrm{C} 6-\mathrm{C} 8-\mathrm{F} 11 & -154.9(7) & \mathrm{C} 18 \mathrm{~A}-\mathrm{C} 19 \mathrm{~A}-\mathrm{C} 20 \mathrm{~A}-\mathrm{C} 21 \mathrm{~A} & -126(2) \\ \text { S4-C6-C8-F11 } & 26.4(9) & \mathrm{C} 14 \mathrm{~A}-\mathrm{N} 1 \mathrm{~A}-\mathrm{C} 22 \mathrm{~A}-\mathrm{C} 23 \mathrm{~A} & 51(4) \\ \mathrm{C} 5-\mathrm{C} 6-\mathrm{C} 8-\mathrm{F} 10 & -38.9(10) & \mathrm{C} 10 \mathrm{~A}-\mathrm{N} 1 \mathrm{~A}-\mathrm{C} 22 \mathrm{~A}-\mathrm{C} 23 \mathrm{~A} & -66(4) \\ \mathrm{S} 4-\mathrm{C} 6-\mathrm{C} 8-\mathrm{F} 10 & 142.4(6) & \mathrm{C} 18 \mathrm{~A}-\mathrm{N} 1 \mathrm{~A}-\mathrm{C} 22 \mathrm{~A}-\mathrm{C} 23 \mathrm{~A} & 172(4) \\ \text { Mo1-O1-C9-O2 } & 178.5(5) & \mathrm{N} 1 \mathrm{~A}-\mathrm{C} 22 \mathrm{~A}-\mathrm{C} 23 \mathrm{~A}-\mathrm{C} 24 \mathrm{~A} & -173(3) \\ \text { Mo1-O1-C9-C9 } & -0.7(8) & \mathrm{C} 22 \mathrm{~A}-\mathrm{C} 23 \mathrm{~A}-\mathrm{C} 24 \mathrm{~A}-\mathrm{C} 25 \mathrm{~A} & -179(4) \\ \end{array}$

Symmetry code: (i) $-x+2,-y,-z+1$. 